ALEA, Lat. Am. J. Probab. Math. Stat. 17, 993-1045 (2020)

DOI: 10.30757/ALEA.v17-36

\title{
Transience and Recurrence of Markov Processes with Constrained Local Time
}

\author{
Adam Barker \\ Department of Mathematics and Statistics, \\ University of Reading, Reading, RG6 6AX, UK \\ E-mail address: adam-barker@outlook.com
}

\begin{abstract}
We study Markov processes conditioned so that their local time must grow slower than a prescribed function. Building upon recent work on Brownian motion with constrained local time in Benjamini and Berestycki (2011); Kolb and Savov (2016), we study transience and recurrence for a broad class of Markov processes.

In order to understand the local time, we determine the distribution of a nondecreasing Lévy process (the inverse local time) conditioned to remain above a given level which varies in time. We study a time-dependent region, in contrast to previous works in which a process is conditioned to remain in a fixed region (e.g. Denisov and Wachtel, 2015; Garbit, 2009), so we must study boundary crossing probabilities for a family of curves, and thus obtain uniform asymptotics for such a family.

Main results include necessary and sufficient conditions for transience or recurrence of the conditioned Markov process. We will explicitly determine the distribution of the inverse local time for the conditioned process, and in the transient case, we explicitly determine the law of the conditioned Markov process. In the recurrent case, we characterise the "entropic repulsion envelope" via necessary and sufficient conditions.
\end{abstract}

\section{Introduction}

We study the asymptotic behaviour of a Markov process whose local time is constrained to grow slower than $f$, an increasing function. The (right-continuous) inverse of the local time process is a subordinator (a non-decreasing Lévy process), so our study of the behaviour of the local time process is effectively equivalent to studying a subordinator conditioned to grow faster than the inverse function $f^{-1}$.

Received by the editors April 24th, 2019; accepted Juen 18th, 2020.

2010 Mathematics Subject Classification. 60J55, 60G51, 60G17, 60J25.

Key words and phrases. Markov Processes, Subordinators, Large Deviations Local Time. Research supported by EPSRC. 
This work is hence related to a number of works on stochastic processes conditioned to remain in a certain fixed region, such as cones in Denisov and Wachtel (2015); Garbit (2009), and Weyl chambers in Denisov and Wachtel (2010); König and Schmid (2010). We highlight the fact that our subordinator is conditioned to remain in a region which varies in time, whereas the aforementioned works consider fixed regions, as appears to be the case for all works prior to Kolb and Savov (2016).

We emphasise that in constraining the local time of a Markov process, the extent to which our constraint affects the process varies over time, depending on the past behaviour of the process. This is a "weak" constraint, in constrast to "strong" constraints such as conditioning a process to avoid a point, where the constraint does not change (see e.g. Bertoin, 1992; Bogdan et al., 2003; Chaumont, 1996). When our conditioned Markov process is recurrent, our constraint varies over all time, whereas when our conditioned Markov process is transient, the constraint varies for only a finite window of time. So our results, especially in the recurrent case, offer a significant contrast to many prior works with a "strong" constraint.

Many works, e.g. Aurzada et al. (2015); Biskup and Louidor (2018); Wachtel and Denisov (2016); Kerkhoff and Lerche (2013); Lerche (1986); Mallein (2016); Pötzelberger and Wang (2001), consider a time-dependent region, and the passage time out of this region is studied. In this paper, we study the boundary crossing probability for a family of curves and study the time at which this crossing occurs (the function $f^{-1}$ forms our boundary of interest). We study these asymptotics, uniformly, among a family of curves in Lemma 4.2 (in contrast to prior non-uniform asymptotic results), and consider the deeper problem of determining the law of a subordinator conditioned to remain in this time-dependent region. Our studies are also similar, in spirit, to various other works on Brownian motion as Kolb and Savov (2017); Roynette et al. (2006); Pitman (1975), Lévy processes Bertoin (1993); Bogdan et al. (2003); Chaumont (1996); Pantí (2017); Yano et al. (2009), and more general diffusions as Çetin (2018); Roynette et al. (2008); Salminen and Vallois (2009) with restricted path behaviour.

Specifically, this work is motivated by previous works on Brownian motion with constrained local time, such as Benjamini and Berestycki (2011), in which a 1dimensional Brownian motion is conditioned so the local time at zero, $\left(L_{t}\right)_{t \geq 0}$, satisfies $L_{t} \leq f(t)$ for all $t \geq 0$, for a given function $f$, and a sufficient condition for transience of the conditioned Brownian motion is found.

In Kolb and Savov (2016), it is shown that the condition is necessary and sufficient for transience of the conditioned Brownian motion, and the law of the conditioned inverse local time process is explicitly determined in both the transient and recurrent cases. In the transient case, an explicit formulation for the conditioned Brownian motion is found, and in the recurrent case the "entropic repulsion envelope" is found.

This paper builds upon Kolb and Savov (2016) in particular, providing analogous results for a much broader class of processes than Brownian motion, with some mild regularity conditions. It was conjectured in Kolb and Savov (2016, Remark 9) that such analogous results hold when the inverse local time process has Lévy measure with regularly varying tail, which we confirm in this paper. We extend beyond this conjecture by including a much more general setting, see Assumption 3.5.

Constraining local time from above imposes weak repellence on a Markov process. Many such processes are studied in works related to polymer physics, see e.g. 
Berger et al. (2018); Caravenna et al. (2012); van der Hofstad and König (2001); van der Hofstad and Klenke (2001); Westwater (1980). Particularly important is the transition between a localised phase, where the polymer remains close to a point, and a delocalised phase, where it moves away.

The goal is often to understand when the transition occurs as underlying model parameters vary, as in e.g. Bolthausen (2002); Berger et al. (2018); Caravenna et al. (2012); den Hollander (1999); Hu et al. (2011). This motivates our study of transience and recurrence of Markov processeses, transience and recurrence corresponding to delocalised and localised phases, respectively.

Now we provide a brief exposition of the main result, before introducing some key definitions.

Main Result. Starting with a recurrent Markov process, we constrain its local time $\left(L_{t}\right)_{t \geq 0}$ so that $L_{t} \leq f(t)$ for all $t$. The following necessary and sufficient condition tells us if the constraint is strong enough to change the process to become transient: it is transient if

$$
\int_{1}^{\infty} f(x) \Pi(d x)<\infty
$$

and the process remains recurrent otherwise, where $\Pi(d x)$ denotes the Lévy measure of the inverse local time subordinator. Our criterion (1.1) can also be understood in terms of the rate of growth of the inverse local time $\left(X_{s}\right)_{s \geq 0}$ as $s \rightarrow \infty$, since it is known (Bertoin, 1996, Theorem III.13) that

$$
\int_{1}^{\infty} f(x) \Pi(d x)<\infty \Longleftrightarrow \lim _{s \rightarrow \infty} \frac{X_{s}}{f^{-1}(s)}=0, \text { almost surely. }
$$

So the boundary choice of $f$, at which the conditioned process changes from recurrent to transient, coincides with the boundary at which $X_{s}$ grows to infinity faster or slower than $f^{-1}(s)$.

The remainder of the paper is structured as follows: Section 2 provides key definitions; Section 3 outlines the statements of the main results and the conditions under which they hold, including the necessary and sufficient conditions for transience/recurrence, the distribution of the conditioned process, and the characterisation of the entropic repulsion envelope; Section 4 contains the proofs of the main results; Sections 5 and 6 contain the proofs of 2 key lemmas required for the main results; Section 7 contains the proofs of the remaining auxiliary lemmas.

\section{Key Definitions}

We shall provide some definitions, following conventions of Bertoin (1996, Chapter IV).

Definition 2.1. A Markov process $\left(M_{t}\right)_{t \geq 0}$, is a $\mathbb{R}^{d}$-valued stochastic process such that for each (almost surely) finite stopping time $T$, under the conditional law $\mathbb{P}\left(\cdot \mid M_{T}=x\right)=\mathbb{P}_{x}(\cdot)$, the shifted process $\left(M_{s+T}\right)_{s \geq 0}$ is independent of $\mathcal{F}_{T}$ and has the same as the law, $\mathbb{P}_{x}$, as the process $M$ started from $x$. Moreover, we impose that $M$ has right-continuous sample paths, $M_{0}=0$, and that the origin is regular and instantaneous. Regular means that for each (almost surely) finite stopping time $T$, if $M_{T}=0$, then inf $\left\{t>T: M_{t}=0\right\}=T$ almost surely. Instantaneous means that for each (almost surely) finite stopping time $T$, if $M_{T}=0$, then $\inf \left\{t>T: M_{t} \neq 0\right\}=$ $T$ almost surely. 
Definition 2.2. For a Markov process, and for an arbitrary choice of $c \in(0, \infty)$, let $l_{1}(x)$ denote the length of the first excursion interval (away from zero) of length $l>x>0$, and define

$$
P(a):= \begin{cases}1 / \mathbb{P}\left(l_{1}(a)>c\right), & 0<a \leq c, \\ \mathbb{P}\left(l_{1}(c)>a\right), & a>c .\end{cases}
$$

Let $g_{n}(a)$ be the start time of the $n$th excursion of length $l>a>0$, and write $N_{a}(t):=\sup \left\{n \in \mathbb{N}: g_{n}(a)<t\right\}$. Then the local time process at zero, $\left(L_{t}\right)_{t \geq 0}$, is defined by $L_{t}:=\lim _{a \rightarrow 0} N_{a}(t) / P(a)$.

A subordinator is defined to be a non-decreasing real-valued stochastic process with stationary independent increments, started from 0 . The right continuous inverse local time, defined by $X_{t}:=\inf \left\{s>0: L_{s}>t\right\}$, is a subordinator. The jumps of $\left(X_{t}\right)_{t \geq 0}$ correspond to excursions of $\left(M_{t}\right)_{t \geq 0}$ away from zero.

The Laplace exponent $\phi$ of a subordinator $X$ is defined by $e^{-\phi(\lambda)}=\mathbb{E}\left[e^{-\lambda X_{1}}\right]$, $\lambda \geq 0$. By the Lévy-Khintchine formula (Bertoin, 1996, p72), $\phi$ can be written

$$
\phi(\lambda)=\mathrm{d} \lambda+\int_{0}^{\infty}\left(1-e^{-\lambda x}\right) \Pi(d x),
$$

where $\mathrm{d}$ is the linear drift, and $\Pi$ is the Lévy measure, which determines the size

and rate of the jumps of $X$, and satisfies $\int_{0}^{\infty}(1 \wedge x) \Pi(d x)<\infty$. We refer to Bertoin (1996) for background on subordinators.

Next, we define some important classes of functions with which we shall work.

Definition 2.3 (Regular Variation and Related Properties).

(i) A function $h: \mathbb{R} \rightarrow \mathbb{R}$ is regularly varying at $\infty$ with index $\alpha \in \mathbb{R}$ if for all $\lambda>0$, we have $\lim _{t \rightarrow \infty} h(\lambda t) / h(t)=\lambda^{\alpha}$. We refer to Bingham et al. (1989) for background on regular variation.

(ii) A function $L: \mathbb{R} \rightarrow \mathbb{R}$ is slowly varying at $\infty$ if $\lim _{t \rightarrow \infty} L(\lambda t) / L(t)=1$ for each $\lambda>0$. A function $h$, regularly varying at $\infty$ of index $\alpha$, can always be written as $h(x)=x^{\alpha} L(x)$, where $L$ is slowly varying at $\infty$, see Bingham et al. (1989).

(iii) The lower index, $\beta(h)$, of a function $h: \mathbb{R} \rightarrow \mathbb{R}$ is the supremum of $\beta \in \mathbb{R}$ for which there exists $C>0$ so that for all $\Lambda>1, h(\lambda x) / h(x) \geq(1+o(1)) C \lambda^{\beta}$, uniformly in $\lambda \in[1, \Lambda]$, as $x \rightarrow \infty$, see Bingham et al. (1989, p68).

(iv) A function $h$ is CRV at $\infty$ if $\lim _{\lambda \rightarrow 1} \lim _{t \rightarrow \infty} h(\lambda t) / h(t)=1$. The class of CRV functions lies between "extended regularly varying" functions and $\mathcal{O}$-regularly varying functions. See Djurčić (1998) for details.

(v) A function $h: \mathbb{R} \rightarrow \mathbb{R}$ is $\mathcal{O}$-regularly varying at $\infty$ if for each $\lambda>0$, both $\limsup \operatorname{su}_{t \rightarrow \infty} h(\lambda t) / h(t)<\infty$ and $\liminf _{t \rightarrow \infty} h(\lambda t) / h(t)>0$. See Djurčić (1998) for further details.

\section{Statements of Main Results}

We aim to constrain the local time so that $L_{t} \leq f(t)$ for all $t \geq 0$, where $f:[0, \infty) \rightarrow(0, \infty)$ is increasing, $f(0) \in(0,1)$, and $\lim _{t \rightarrow \infty} f(t)=\infty$. This work 
concerns the behaviour of our process as $t \rightarrow \infty$, which is unaffected by the condition on $f(0)$. Before stating our main results, we define the regularity conditions under which these results hold.

3.1. Regularity Conditions. We shall impose regularity conditions on the function $f$, its inverse function $g:=f^{-1}$ (extended so that for $x \in[0, f(0)), g(x)=0$ ), and the tail $\bar{\Pi}(x):=\Pi(x, \infty)$ in two main cases of interest. Our conditions are imposed on the inverse local time subordinator rather than directly on the Markov process. Now let us define the main cases of interest for our results:

Assumption 3.1 (Case (i)). We impose on our subordinator that the drift is zero, and the tail $\bar{\Pi}(x)=\Pi(x, \infty)$ is regularly varying at $\infty$ with index $-\alpha \in(-1,0)$, so $\bar{\Pi}(x)=x^{-\alpha} L(x)$ for $L$ slowly varying at $\infty$. We further impose there exist $B, N>0$ such that the function $x \mapsto x^{N} L(x)$ is non-decreasing on $(B, \infty)$.

We impose that $f(0) \in(0,1), \lim _{t \rightarrow \infty} f(t)=\infty, f$ is differentiable, $t f^{\prime}(t) \bar{\Pi}(t)$ decreases to 0 as $t \rightarrow \infty$ (so $f$ is increasing), the inverse $g:=f^{-1}$ satisfies $\lim _{t \rightarrow \infty} g(t+$ $\varepsilon) / g(t)=1$ for all $\varepsilon>0$, and there exists some value $\beta>(1+2 \alpha) /\left(2 \alpha+\alpha^{2}\right)>1$ such that

$$
\lim _{t \rightarrow \infty} t \bar{\Pi}\left(\frac{g(t)}{\log (t)^{\beta}}\right)=0 .
$$

Remark 3.2. Case (i) includes stable subordinators, and subordinators whose Lévy measure has similarly well-behaved tail asymptotics. Thus the set of Markov processes corresponding to case (i) includes Bessel processes, stable Lévy processes of index $\alpha \in(1,2)$, and other Markov processes with similarly well-behaved asymptotics. Case (ii) corresponds to a much broader class of processes.

Assumption 3.3 (Case (ia)). Under the assumptions of case (i), define "case (ia)" by imposing $f, f^{\prime}$ are $\mathcal{O}$-regularly varying at $\infty$, the densities $f_{t}(x) d x:=\mathbb{P}\left(X_{t} \in d x\right)$ and $u(x) d x:=\Pi(d x)$ exist, $u$ has bounded increase and bounded decrease (see Bingham et al. (1989, p71) for precise definitions), and there exist constants $a, x_{0} \in$ $(0, \infty)$, such that for all $t \in(0, \infty)$ and $x \geq g(t)+x_{0}$, where $g=f^{-1}$,

$$
f_{t}(x) \leq \operatorname{atu}(x) \text {. }
$$

Remark 3.4. If $\bar{\Pi}$ is regularly varying at $\infty$ and the density $f_{t}$ exists, then (3.2) holds for each fixed $t$ and $x>x(t)$, where $x(t)$ may depend on $t$ (see e.g. Yakymiv, 2002, Theorem 1). Here we further impose a bound on $x(t)$, so that (3.2) holds uniformly among sufficiently many $x$ and $t$ for us to prove Theorem 3.18. For a stable subordinator of index $\alpha \in(0,1)$, the density $f_{t}$ exists (see Bertoin, 1996, p227) and (3.2) holds (see Corollary 3.19), so case (ia) includes stable subordinators.

Assumption 3.5 (Case (ii)). We impose on our subordinator that the drift is zero, and the tail function $\bar{\Pi}(x)=\Pi(x, \infty)$ is CRV at $\infty$, with lower index $\beta(\bar{\Pi})>-1$. We impose that $f(0) \in(0,1)$, for $f$ increasing, and that there exists $\varepsilon>0$ such that for $g:=f^{-1}$,

$$
\lim _{t \rightarrow \infty} t^{1+\varepsilon} \bar{\Pi}(g(t))=0
$$

Remark 3.6. In Assumption 3.5, we impose $\beta(\bar{\Pi})>-1$, which is equivalent to imposing that the function $\int_{0}^{x} \bar{\Pi}(y) d y$ has positive increase as $x \rightarrow \infty$. This has many equivalent formulations Bertoin (1996, Ex. III.7), Bingham et al. (1989, Section 2.1), and appears naturally in a range of contexts Barker (2019, p2), Bertoin (1996, p87). 
Now let us introduce some notation required to formulate our results. Recall that $f:[0, \infty) \rightarrow(0, \infty)$ is increasing, $f(0) \in(0,1)$, and $g:=f^{-1}$ is the inverse of $f$, where we take $g(x)=0$ for $x \in[0, f(0))$. The event $\mathcal{O}_{u}$ corresponds to bounding the inverse local time until time $u$ (or equivalently, bounding the local time until time $g(u))$. We will study the asymptotics of $\mathbb{P}\left(\mathcal{O}_{u}\right)$ as $u \rightarrow \infty$, and those of the integral $\Phi(s)$ of this probability.

$$
\begin{aligned}
\mathcal{O}_{u} & :=\left\{X_{s} \geq g(s), \forall 0 \leq s \leq u\right\}, \\
\Phi(s) & :=\int_{0}^{s} \mathbb{P}\left(\mathcal{O}_{u}\right) d u
\end{aligned}
$$

We also study the event $\mathcal{O}_{u}$ for the process $X^{(0, a)}$ with truncated Lévy measure $\Pi(d x) \mathbb{1}_{\{x \in(0, a)\}}$,

$$
\mathcal{O}_{u, X^{(0, a)}}:=\left\{X_{s}^{(0, a)} \geq g(s), \forall 0 \leq s \leq u\right\}
$$

The time of our subordinator's first jump of size larger than $x>0$, or in the interval $(a, b)$ for $b>a>0$, are respectively denoted by

$$
\begin{array}{ll}
\Delta_{1}^{x} & :=\inf \left\{t \geq 0: X_{t}-X_{t-}>x\right\}, \\
\Delta_{1}^{(a, b)} & :=\inf \left\{t \geq 0: X_{t}-X_{t-} \in(a, b)\right\} .
\end{array}
$$

In Proposition 3.10 and Proposition 3.15, we determine that $I(f)<\infty$ is a necessary and sufficient condition for transience of the conditioned process, where

$$
I(f):=\int_{1}^{\infty} f(x) \Pi(d x) .
$$

Remark 3.7. The necessary and sufficient condition $I(f)<\infty$ arises naturally in a number of contexts, including rate of growth of subordinators (Bertoin, 1996, Theorem III.13) and spectrally negative Lévy processes (Pardo, 2008, Theorem 3).

For $h<t$, by the stationary independent increments property, $\left\{X_{t}>g(t) \mid X_{h}=\right.$ $y\}$ is effectively equivalent to $\left\{X_{t-h}>g(t)-y\right\}=\left\{X_{t-h}>g((t-h)+h)-y\right\}$, in terms of probability. The new boundary for $X$ to stay above is given by $g_{y}^{h}(\cdot)$, with $\mathcal{O}_{u}^{g_{y}^{h}}, \Phi_{y}^{h}(s)$ corresponding to $\mathcal{O}_{u}, \Phi(s)$, where

$$
\begin{aligned}
g_{y}^{h}(t) & :=g(t+h)-y, \\
\mathcal{O}_{u}^{g_{y}^{h}} & :=\left\{X_{s} \geq g_{y}^{h}(s), \forall 0 \leq s \leq u\right\}, \\
\Phi_{y}^{h}(s) & :=\int_{0}^{s} \mathbb{P}\left(\mathcal{O}_{u}^{g_{y}^{h}}\right) d u .
\end{aligned}
$$

The functions $\rho(\cdot), \rho_{y}^{h}(\cdot)$ are error terms in the upcoming ODEs (3.16) and (3.17).

$$
\begin{aligned}
\rho(t) & :=\frac{\mathbb{P}\left(\mathcal{O}_{t}\right)}{\Phi(t)}-\bar{\Pi}(g(t)), \\
\rho_{y}^{h}(t) & :=\frac{\mathbb{P}\left(\mathcal{O}_{t}^{g_{y}^{h}}\right)}{\Phi_{y}^{h}(t)}-\bar{\Pi}\left(g_{y}^{h}(t)\right) .
\end{aligned}
$$


The law of our conditioned process will be found by taking limits. Recalling the notation (3.4) and (3.11), for the measure $\mathbb{Q}(\cdot):=\lim _{t \rightarrow \infty} \mathbb{P}\left(\cdot \mid \mathcal{O}_{t}\right)$, for all $\mathcal{B}_{h} \subseteq$ $\mathcal{O}_{h}, \mathcal{B}_{h} \in \mathcal{F}_{h}$, where $\left(\mathcal{F}_{u}\right)_{u \geq 0}$ is the natural filtration of $X$,

$$
\begin{aligned}
\mathbb{Q}\left(X_{h} \in d y ; \mathcal{B}_{h}\right): & =\lim _{t \rightarrow \infty} \mathbb{P}\left(X_{h} \in d y ; \mathcal{B}_{h} \mid \mathcal{O}_{t}\right) \\
& =\lim _{t \rightarrow \infty} \frac{\mathbb{P}\left(\mathcal{O}_{t} \mid X_{h} \in d y ; \mathcal{B}_{h}\right) \mathbb{P}\left(X_{h} \in d y ; \mathcal{B}_{h}\right)}{\mathbb{P}\left(\mathcal{O}_{t}\right)} \\
& =\mathbb{P}\left(X_{h} \in d y ; \mathcal{B}_{h}\right) \lim _{t \rightarrow \infty} \frac{\mathbb{P}\left(\mathcal{O}_{t-h}^{g_{y}^{h}}\right)}{\mathbb{P}\left(\mathcal{O}_{t}\right)} .
\end{aligned}
$$

We shall see in Theorems 3.8 and 3.14 that the limit in (3.15) exists and is finite. In order to understand the behaviour of $X$ under $\mathbb{Q}$, we study the probabilities $\mathbb{P}\left(\mathcal{O}_{t}^{g_{y}^{h}}\right)$ and $\mathbb{P}\left(\mathcal{O}_{t}\right)$ as $t \rightarrow \infty$. Corollary 4.4 relates the asymptotics of $\mathbb{P}\left(\mathcal{O}_{t}\right)$ to $\Phi(t)$, and $\mathbb{P}\left(\mathcal{O}_{t}^{g_{y}^{h}}\right)$ to $\Phi_{y}^{h}(t)$. We obtain the ODEs

$$
\begin{aligned}
\mathbb{P}\left(\mathcal{O}_{t}\right) & =\frac{d}{d t} \Phi(t)=(\bar{\Pi}(g(t))+\rho(t)) \Phi(t), \\
\mathbb{P}\left(\mathcal{O}_{t}^{g_{y}^{h}}\right) & =\frac{d}{d t} \Phi_{y}^{h}(t)=\left(\bar{\Pi}\left(g_{y}^{h}(t)\right)+\rho_{y}^{h}(t)\right) \Phi_{y}^{h}(t) .
\end{aligned}
$$

These ODEs are easily solved, yielding (for $B>0$ as in Assumption 3.1) the expressions

$$
\begin{aligned}
\Phi(t) & =\Phi(1) \exp \left(\int_{1}^{t} \bar{\Pi}(g(s)) d s+\int_{1}^{t} \rho(s) d s\right), \\
\Phi_{y}^{h}(t) & =\Phi_{y}^{h}\left(t_{0}(y)\right) \exp \left(\int_{t_{0}(y)}^{t} \bar{\Pi}\left(g_{y}^{h}(s)\right) d s+\int_{t_{0}(y)}^{t} \rho_{y}^{h}(s) d s\right), \\
t_{0}(y) & :=f(A y) \vee f(1+2 / A), \quad A>3 \vee(B-1) .
\end{aligned}
$$

The error terms $\rho(\cdot)$ and $\rho_{y}^{h}(\cdot)$ are later shown to be integrable (the latter uniformly in $y$ and $h$ ), which is key for determining the distribution of our conditioned process. The required bound for $\rho(\cdot)$ is given in Remark 4.5, and we provide a uniform bound for $\rho_{y}^{h}(\cdot)$ in Lemma 4.2, proven in Section 5 .

3.2. Results in the $I(f)<\infty$ Case. We shall see in Theorem 3.12 that when $I(f)<$ $\infty$, any possible weak limit of our conditioned process $M$ is transient. Theorem 3.8 finds the distribution of the process $X$ in this case.

Theorem 3.8. With assumptions on $f$ and $\bar{\Pi}$ in case (i) or case (ii), if $I(f)<\infty$, then the measure $\mathbb{Q}(\cdot):=\lim _{t \rightarrow \infty} \mathbb{P}\left(\cdot \mid \mathcal{O}_{t}\right)$ exists for the space $\mathcal{D}[0, \infty)$, to which $X$ belongs, of càdlàg paths on $[0, \infty)$, in the sense that for all $h>0, y>g(h)$, for all $\mathcal{B}_{h} \subseteq \mathcal{O}_{h}, \mathcal{B}_{h} \in \mathcal{F}_{h}$, where $\left(\mathcal{F}_{u}\right)_{u \geq 0}$ is the natural filtration of $X$,

$$
\mathbb{Q}\left(X_{h} \in d y ; \mathcal{B}_{h}\right)=\frac{\Phi_{y}^{h}(\infty)}{\Phi(\infty)} \mathbb{P}\left(X_{h} \in d y ; \mathcal{B}_{h}\right),
$$

where $\Phi(\infty)<\infty, \Phi_{y}^{h}(\infty)<\infty$. Define, independently of $M$ or $X$, the random variable $\mathfrak{C}$ by

$$
\mathbb{P}(\mathfrak{C} \in d s):=\frac{\mathbb{P}\left(\mathcal{O}_{s}\right)}{\Phi(\infty)} d s, \quad s \geq 0
$$


which exists since $\Phi(\infty)<\infty$. Then for all $h \geq 0, \mathbb{Q}\left(X_{h}<\infty\right)=\mathbb{P}(\mathfrak{C}>h)$.

Remark 3.9. Since $\mathbb{Q}\left(X_{h}<\infty\right)=\mathbb{P}(\mathfrak{C}>h)$ for all $h>0, X$ under $\mathbb{Q}$ is finite until a random time, which we denote by $T_{\infty}$, and which has the same distribution under $\mathbb{Q}$ as $\mathfrak{C}$ under $\mathbb{P}$. In particular, $\mathbb{Q}\left(T_{\infty} \in d s\right)=\mathbb{P}(\mathfrak{C} \in d s)$ for all $s \geq 0$. In Theorem 3.12, we will show that under any possible weak limit measure, the process $M$ never returns to 0 after time $X_{T_{\infty}-}=\lim _{s \uparrow T_{\infty}} X_{s}$.

We shall now determine the behaviour of the conditioned Markov process $M$ until its first excursion longer than $g(t)$, and the time at which this excursion occurs. Describing the behaviour until the first excursion longer than $g(t)$, as $t \rightarrow \infty$, in fact gives full knowledge of how $M$ behaves under $\mathbb{Q}$ until the time of its final, infinite excursion. We verify in Proposition 3.11 that $M$ is transient under $\mathbb{Q}$ by showing that the process $M$ never returns to 0 after the start of this final excursion. Remark 3.13 considers the behaviour after this time.

Proposition 3.10. In cases (i) and (ii), $\left(\left(X_{u}\right)_{\Delta_{1}^{g(t)}>u \geq 0}, \Delta_{1}^{g(t)}\right), t \geq 0$ under $\mathbb{P}\left(\cdot \mid \mathcal{O}_{t}\right)$ converges as $t \rightarrow \infty$, in the sense that there exists a unique limit measure $\mathbb{Q}^{\prime}(\cdot)$, on the space $\mathcal{D}[0, \infty) \times(0, \infty)$, such that for all $y>g(x), t>b>a>x>0$, with $a, b, x, y$ fixed, and for all events $\mathcal{B}_{X} \in \mathcal{F}_{x}$, where $\left(\mathcal{F}_{u}\right)_{u \geq 0}$ is the natural filtration of $X$, such that $\mathcal{B}_{X} \subseteq \mathcal{O}_{x}$, for $\mathfrak{C}$ as in (3.21),

$$
\begin{aligned}
\lim _{t \rightarrow \infty} \mathbb{P}\left(X_{x} \in d y ; \mathcal{B}_{X} ; \Delta_{1}^{g(t)} \in(a, b) \mid \mathcal{O}_{t}\right) & =\int_{a}^{b} \mathbb{P}\left(X_{x} \in d y ; \mathcal{B}_{X} \mid \mathcal{O}_{s}\right) \mathbb{P}(\mathfrak{C} \in d s) \\
& =: \mathbb{Q}^{\prime}\left(X_{x} \in d y ; \mathcal{B}_{X} ; T_{\infty}^{\prime} \in(a, b)\right),
\end{aligned}
$$

for the explosion time $T_{\infty}^{\prime}$ defined under $\mathbb{Q}^{\prime}$ as $T_{\infty}$ is defined under $\mathbb{Q}$ in Remark 3.9 . The projection of $\mathbb{Q}^{\prime}$ onto $(0, \infty)$ agrees with $\mathbb{Q}$ in the sense that $\mathbb{Q}^{\prime}\left(T_{\infty}^{\prime} \in d s\right)=$ $\mathbb{Q}\left(T_{\infty} \in d s\right)=\mathbb{P}(\mathfrak{C} \in d s)$.

We shall now determine the behaviour of the conditioned process $M$ until a time corresponding to the point at which $X$ becomes infinite. Theorem 3.12 and Remark 3.13 consider the behaviour after this time. Proposition 3.11 requires some understanding of excursion theory of Markov processes. For background on excursion theory, we direct the reader to Bertoin (1996, Chapter IV).

Proposition 3.11. In cases (i) and (ii), if $I(f)<\infty$ then there exists a measure $\mathbb{Q}^{\prime \prime}(\cdot)$ on the product space of the space containing the excursion process with $\mathcal{D}[0, \infty) \times(0, \infty)$, such that for all fixed $b>a>h>0$, and for $\mathcal{B} \subseteq \mathcal{O}_{h}$, $\mathcal{B} \in \mathcal{F}_{h}$, where $\mathcal{F}$ denotes the natural filtration of $X$, with $F_{1}$ a bounded continuous functional on the excursion process $\left(\varepsilon_{s}\right)_{s \geq 0}$ of $M$, defining the operator $\pi_{h}\left(\left(Z_{u}\right)_{u \geq 0}\right):=\left(Z_{u}\right)_{h \geq u \geq 0}$, and letting $F_{1}$ satisfy $\left.F_{1}\left(\left(\varepsilon_{s}\right)_{s \geq 0}\right)=F_{1}\left(\pi_{h}\left(\left(\varepsilon_{s}\right)_{s \geq 0}\right)\right)\right)$, so $F_{1}$ depends only on the excursion process of $M$ up to time $h$, we have

$$
\begin{aligned}
& \lim _{t \rightarrow \infty} \mathbb{E}\left[F_{1}\left(\pi_{h}\left(\left(\varepsilon_{s}\right)_{s \geq 0}\right)\right) \mathbb{1}_{\left\{\pi_{h}(X) \in \mathcal{B}\right\}} \mathbb{1}_{\left\{\Delta_{1}^{g(t)} \in(a, b)\right\}} \mid \mathcal{O}_{t}\right] \\
& =\int_{\nu \in \mathcal{B}} \int_{u \in(a, b)} \mathbb{E}\left[F_{1}\left(\pi_{h}\left(\left(\varepsilon_{s}\right)_{s \geq 0}\right)\right) \mid \pi_{h}(X)=\nu\right] \mathbb{Q}^{\prime}\left(\pi_{h}(X) \in d \nu ; T_{\infty}^{\prime} \in d u\right) \\
& =: \mathbb{E}_{\mathbb{Q}^{\prime \prime}}\left[F_{1}\left(\pi_{h}\left(\left(\varepsilon_{s}\right)_{s \geq 0}\right)\right) \mathbb{1}_{\left\{\pi_{h}(X) \in \mathcal{B}\right\}} \mathbb{1}_{\left\{T_{\infty}^{\prime \prime} \in(a, b)\right\}},\right.
\end{aligned}
$$

where $T_{\infty}^{\prime \prime}$ is the explosion time under the measure $\mathbb{Q}^{\prime \prime}(\cdot)$, and the projection of $\mathbb{Q}^{\prime \prime}(\cdot)$ onto $\mathcal{D}[0, \infty) \times(0, \infty)$ agrees with $\mathbb{Q}^{\prime}(\cdot)$. In particular, with the notation $\Delta:=\Delta_{1}^{g(t)},\left(\left(M_{t}\right)_{X_{\Delta-}>t \geq 0},\left(X_{s}\right)_{\Delta>s \geq 0}, \Delta\right)$ under $\mathbb{P}\left(\cdot \mid \mathcal{O}_{t}\right)$ converges weakly as $t \rightarrow$ 


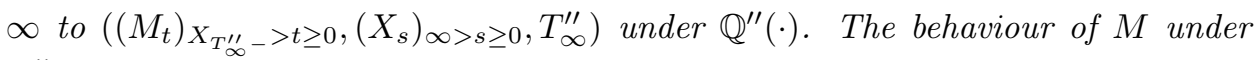
$\mathbb{Q}^{\prime \prime}(\cdot)$ before time $X_{T_{\infty}^{\prime \prime}-}$ has the same distribution as the following construction, expressed in terms of the original measure $\mathbb{P}$ as follows: sampling the random time $\mathfrak{C}=s$ under $\mathbb{P}(\cdot)$, we run $X$ conditioned on $\mathcal{O}_{s}$ until time $s$, take $X_{u}=\infty$ for all $u \geq s$, then construct $M$ via its excursions using $\left(X_{u}\right)_{\infty>u \geq 0}$ to determine the timing and length of each excursion, where we sample each excursion of $M$ until time $X_{s-}$ using the excursion measure conditional on the given excursion length.

Theorem 3.12. In cases (i) and (ii), if $I(f)<\infty$ then $M$ is transient under any possible weak limit of the measure $\mathbb{P}\left(\cdot \mid \mathcal{O}_{t}\right)$ as $t \rightarrow \infty$.

Remark 3.13. While the last excursion of the Markov process $M$ is not dealt with explicitly here, the behaviour of $M$ from time $X_{\mathfrak{C}-}$ onwards should be the same as that of $M$ conditioned to avoid zero. Proving this requires existence of the limit as $g(t) \rightarrow \infty$ of the excursion measure conditioned on the length (lifetime) of an excursion being longer than $g(t)$, which is beyond the scope of this work. This is verified in the simple, single case where $M$ is a 1-dimensional Brownian motion in Kolb and Savov (2016, p8). When $M$ is a Lévy process, the behaviour of the process conditioned to avoid zero is well understood, see Pantí (2017, Theorem 8). There is some technical difficulty in applying results from Pantí (2017) to our final excursion. The measures $\mathbb{Q}, \mathbb{Q}^{\prime}, \mathbb{Q}^{\prime \prime}$ are constructed by conditioning until a deterministic time $t \rightarrow \infty$, but in Pantí (2017), the measure is constructed by conditioning until an independent exponential random time with parameter $q \rightarrow 0$. Equivalence of such deterministic and random limits is a separate matter, beyond the scope of this work.

3.3. Results in the $I(f)=\infty$ Case. We now restrict our attention to case (i). We will see that when $I(f)=\infty$, our conditioned Markov process is recurrent. Theorem 3.14 finds the distribution of the conditioned inverse local time subordinator in this case.

Theorem 3.14. In case (i), if $I(f)=\infty$, then the law $\mathbb{Q}(\cdot)=\lim _{t \rightarrow \infty} \mathbb{P}\left(\cdot \mid \mathcal{O}_{t}\right)$ exists for the process $X$ in the sense that for all $h>0$ and $y \geq g(h)$, for all $h>0$, $y>g(h)$, for all $\mathcal{B}_{h} \subseteq \mathcal{O}_{h}, \mathcal{B}_{h} \in \mathcal{F}_{h}$, where $\left(\mathcal{F}_{u}\right)_{u \geq 0}$ is the natural filtration of $X$,

$$
\begin{aligned}
\mathbb{Q}\left(X_{h} \in d y ; \mathcal{B}_{h}\right) & =\lim _{t \rightarrow \infty} \frac{\mathbb{P}\left(\mathcal{O}_{t-h}^{g_{y}^{h}}\right)}{\mathbb{P}\left(\mathcal{O}_{t}\right)} \mathbb{P}\left(X_{h} \in d y ; \mathcal{B}_{h}\right) \\
& =: q_{h}(y) \mathbb{P}\left(X_{h} \in d y ; \mathcal{B}_{h}\right),
\end{aligned}
$$

where $q_{h}(y)$ is finite, non-decreasing in $y$, and satisfies

$$
\begin{gathered}
q_{h}(y)=\frac{\Phi_{y}^{h}\left(t_{0}(y)\right)}{\Phi(1)} \exp \left(\int_{t_{0}(y)}^{\infty}\left(\bar{\Pi}\left(g_{y}^{h}(s)\right)-\bar{\Pi}(g(s))+\rho_{y}^{h}(s)\right) d s\right. \\
\left.-\int_{1}^{\infty} \rho(s) d s-\int_{1}^{t_{0}(y)} \bar{\Pi}(g(s)) d s\right), \\
t_{0}(y):=f(A y) \vee f(1+2 / A), \quad A>3 \vee(B-1),
\end{gathered}
$$

for $B>0$ as in Assumption 3.1, and where each integral is finite. In the case that $t_{0}(y)<1$, the final integral should be interpreted as $-\int_{1}^{t_{0}(y)} \bar{\Pi}(g(s)) d s=$ $\int_{t_{0}(y)}^{1} \bar{\Pi}(g(s)) d s$. 
We now verify that when $I(f)=\infty, M$ is recurrent under the new measure $\mathbb{Q}^{\prime \prime}(\cdot)$, as $X$ never hits infinity at a finite time, $\mathbb{Q}$-almost surely, and then $M$ under $\mathbb{Q}^{\prime \prime}(\cdot)$ is constructed from its excursion process and $X$.

Proposition 3.15. In case (i), if $I(f)=\infty$, then for each $h>0$,

$$
\mathbb{Q}\left(X_{h} \in(g(h), \infty)\right)=1 .
$$

Proposition 3.16. In case (i), if $I(f)=\infty$, then there exists a measure $\mathbb{Q}^{\prime \prime}(\cdot)$ on the product space of the space containing the excursion process with the space $\mathcal{D}[0, \infty)$ of càdlàg paths on $[0, \infty)$, such that for all fixed $h>0$, and for $\mathcal{B} \subseteq$ $\mathcal{O}_{h}, \mathcal{B} \in \mathcal{F}_{h}$, where $\mathcal{F}$ denotes the natural filtration of $X$, let $F_{1}$ be a bounded continuous functional on the excursion process $\left(\varepsilon_{s}\right)_{s \geq 0}$ of $M$, defining the operator $\pi_{h}\left(\left(Z_{u}\right)_{u \geq 0}\right):=\left(Z_{u}\right)_{h \geq u \geq 0}$, with $F_{1}$ such that $\left.F_{1}\left(\left(\varepsilon_{s}\right)_{s \geq 0}\right)=F_{1}\left(\pi_{h}\left(\left(\varepsilon_{s}\right)_{s \geq 0}\right)\right)\right)$,

$$
\begin{aligned}
& \lim _{t \rightarrow \infty} \mathbb{E}\left[F_{1}\left(\pi_{h}\left(\left(\varepsilon_{s}\right)_{s \geq 0}\right)\right) \mathbb{1}_{\left\{\pi_{h}(X) \in \mathcal{B}\right\}} \mid \mathcal{O}_{t}\right] \\
& =\int_{\nu \in \mathcal{B}} \mathbb{E}\left[F_{1}\left(\pi_{h}\left(\left(\varepsilon_{s}\right)_{s \geq 0}\right)\right) \mid \pi_{h}(X)=\nu\right] \mathbb{Q}\left(\pi_{h}(X) \in d \nu\right) \\
& =: \mathbb{E}_{\mathbb{Q}^{\prime \prime}}\left[F_{1}\left(\pi_{h}\left(\left(\varepsilon_{s}\right)_{s \geq 0}\right)\right) \mathbb{1}_{\left\{\pi_{h}(X) \in \mathcal{B}\right\}}\right]
\end{aligned}
$$

where the projection of $\mathbb{Q}^{\prime \prime}(\cdot)$ onto $\mathcal{D}[0, \infty)$ agrees with $\mathbb{Q}(\cdot)=\lim _{t \rightarrow \infty} \mathbb{P}\left(\cdot \mid \mathcal{O}_{t}\right)$. In particular, as $t \rightarrow \infty,\left(\left(M_{t}\right)_{\infty>t \geq 0},\left(X_{s}\right)_{\infty>s \geq 0}\right)$ under $\mathbb{P}\left(\cdot \mid \mathcal{O}_{t}\right)$ converges weakly to $\left(\left(M_{t}\right)_{\infty>t \geq 0},\left(X_{s}\right)_{\infty>s \geq 0}\right)$ under $\mathbb{Q}^{\prime \prime}(\cdot)$. We construct $M$ via its excursions using $\left(X_{u}\right)_{\infty>u \geq 0}$ to determine the timing and length of each excursion, where we sample the excursions of $M$ using the excursion measure conditional on each excursion length. Moreover, $M$ visits 0 at arbitrarily large times, so $M$ is recurrent under $\mathbb{Q}^{\prime \prime}(\cdot)$.

Now we shall determine the entropic repulsion envelope through Theorem 3.18.

Definition 3.17. A non-decreasing function $w$, with $\lim _{h \rightarrow \infty} w(h)=\infty$, is in the entropic repulsion envelope $R_{g}$ (for the function $g=f^{-1}$ ) if

$$
\lim _{h \rightarrow \infty} \mathbb{Q}^{\prime \prime}\left(X_{h} \geq w(h) g(h)\right)=1 .
$$

Theorem 3.18. In case (ia), a necessary and sufficient condition for nondecreasing $w$, for which $\lim _{h \rightarrow \infty} w(h)=\infty$, to be in $R_{g}$ (for the function $g=f^{-1}$ ) is

$$
w \in R_{g} \Longleftrightarrow \lim _{h \rightarrow \infty} \int_{h}^{f(w(h) g(h))} \bar{\Pi}(g(s)) d s=0 .
$$

As a result, one can verify that the entropic repulsion envelope is always non-empty in case (ia), by finding suitable $w$. We illustrate the generality of Theorem 3.18 via the following corollary, expanding upon Kolb and Savov (2016, Theorem 4).

Corollary 3.19. In case (i), with $f, f^{\prime} \mathcal{O}$-regularly varying at $\infty$, for a stable subordinator of index $\alpha \in(0,1)$, a necessary and sufficient condition for non-decreasing $w$, with $\lim _{h \rightarrow \infty} w(h)=\infty$, to be in $R_{g}$, for $g=f^{-1}$ is

$$
w \in R_{g} \Longleftrightarrow \lim _{h \rightarrow \infty} \int_{h}^{f(w(h) g(h))} g(s)^{-\alpha} d s=0 .
$$




\section{Proofs of Main Results}

This section contains the proofs of the results stated in Section 3. First we state Lemmas 4.2 and 4.3, which are proven in Sections 5 and 7, respectively.

Definition 4.1. In this paper we use the following asymptotic notation:

$f(x) \sim g(x)$ as $x \rightarrow \infty$ if $\lim _{x \rightarrow \infty} f(x) / g(x)=1$.

$f(x) \lesssim g(x)$ if there exists $C \in(0, \infty)$ such that for all large enough $x, f(x) \leq$ $C g(x)$.

Moreover, we write $f(x) \gtrsim g(x)$ if $g(x) \lesssim f(x)$, and

$f(x) \asymp g(x)$ if both $f(x) \gtrsim g(x)$ and $f(x) \lesssim g(x)$.

Lemma 4.2. In cases (i) and (ii), there exists a function $u(t)$ with $\lim _{t \rightarrow \infty} u(t)=$ 0 , and there exists $\varepsilon>0$ such that for all $A>3$, uniformly in $h>0, y>g(h)$, and $t>t_{0}(y)$ as defined in (3.20),

$$
\begin{aligned}
& \rho_{y}^{h}(t) \lesssim \frac{1}{t \log (t)^{1+\varepsilon}}\left(1+\frac{1}{f(y)-h}\right), \\
& \rho_{y}^{h}(t) \leq u(t) \bar{\Pi}(g(t))\left(1+\frac{1}{f(y)-h}\right) .
\end{aligned}
$$

The inequalities (4.1) and (4.2) also hold when $y=h=0$, for $t>t_{0}(0)>0$, with $\rho(t)$ in place of $\rho_{y}^{h}(t)$.

Lemma 4.3. In cases (i) and (ii), for the function $\rho$ as defined in (3.13), $\rho(t)=$ $o\left(\bar{\Pi}(g(t))\right.$ as $t \rightarrow \infty$, and moreover, $\int_{1}^{\infty} \rho(s) d s>-\infty$.

Corollary 4.4 follows immediately from Lemmas 4.2 and 4.3 .

Corollary 4.4. In cases (i) and (ii), as $t \rightarrow \infty$,

$$
\mathbb{P}\left(\mathcal{O}_{t}\right)=(\bar{\Pi}(g(t))+\rho(t)) \Phi(t)=(1+o(1)) \bar{\Pi}(g(t)) \Phi(t) .
$$

Remark 4.5. Taking $y=h=0$ in (4.1), it follows that $\int_{1}^{\infty} \rho(s) d s<\infty$. Then as $\int_{1}^{\infty} \rho(s) d s>-\infty$ by Lemma 4.3, it follows immediately from (3.18) that as $t \rightarrow \infty$,

$$
\Phi(t)=\Phi(1) \exp \left(\int_{1}^{t}(\bar{\Pi}(g(s))+\rho(s)) d s\right) \asymp \exp \left(\int_{1}^{t} \bar{\Pi}(g(s)) d s\right) .
$$

4.1. Proofs in the $I(f)<\infty$ Case.

\subsubsection{Proof of Theorem 3.8.}

Proof of Theorem 3.8: First, let us verify that $\Phi(\infty)<\infty$. Recalling that $g:=f^{-1}$, we have

$$
\begin{aligned}
I(f) & =\int_{1}^{\infty} f(x) \Pi(d x)=\int_{1}^{\infty} \int_{0}^{f(x)} d y \Pi(d x) \\
& =\int_{0}^{\infty} \int_{1 \vee g(y)}^{\infty} \Pi(d x) d y=\int_{0}^{\infty} \bar{\Pi}(1 \vee g(y)) d y .
\end{aligned}
$$

Now, recall from (3.18) that

$$
\Phi(\infty)=\Phi(1) \exp \left(\int_{1}^{\infty} \bar{\Pi}(g(s)) d s+\int_{1}^{\infty} \rho(s) d s\right) .
$$


By Corollary 4.4, as $s \rightarrow \infty, \rho(s)=o(\bar{\Pi}(g(s)))$. Then by (4.4), since $I(f)<\infty$,

$$
\int_{1}^{\infty} \bar{\Pi}(g(s)) d s+\int_{1}^{\infty} \rho(s) d s \stackrel{4.4}{\lesssim} \int_{1}^{\infty} \bar{\Pi}(g(s)) d s \stackrel{(4.4)}{<} \infty,
$$

so $\Phi(\infty)<\infty$. Now, $\int_{1}^{\infty} \rho(s) d s>-\infty$ by Lemma 4.3 , and hence

$$
I(f)<\infty \Longleftrightarrow \Phi(\infty)<\infty .
$$

To show $\Phi_{y}^{h}(\infty)<\infty$, with $t_{0}(y)$ as defined in (3.20), recall that by (3.19),

$$
\Phi_{y}^{h}(\infty)=\Phi_{y}^{h}\left(t_{0}(y)\right) \exp \left(\int_{t_{0}(y)}^{\infty} \bar{\Pi}\left(g_{y}^{h}(s)\right) d s+\int_{t_{0}(y)}^{\infty} \rho_{y}^{h}(s) d s\right) .
$$

Now, observe that for each fixed $y, h>0, g(s) \sim g_{y}^{h}(s)$ as $s \rightarrow \infty$, by (3.10) and the properties of $g$ introduced in Assumption 3.1, so $\bar{\Pi}(g(s)) \sim \bar{\Pi}\left(g_{y}^{h}(s)\right)$ as $s \rightarrow \infty$, since $\bar{\Pi}$ is CRV at $\infty$. Now, applying (4.2) and (4.4), since $y$ and $h$ are fixed and $y>g(h)$ implies $f(y)-h>0$, noting $s>t_{0}(y)$ ensures $g_{y}^{h}(s)>0$, we get

$$
\int_{t_{0}(y)}^{\infty} \bar{\Pi}\left(g_{y}^{h}(s)\right) d s+\int_{t_{0}(y)}^{\infty} \rho_{y}^{h}(s) d s \stackrel{(4.2)}{\lesssim}\left(1+\frac{1}{f(y)-h}\right) \int_{t_{0}(y)}^{\infty} \bar{\Pi}(g(s)) d s \stackrel{(4.4)}{<} \infty,
$$

so $\Phi_{y}^{h}(\infty)<\infty$. By (3.15) and Corollary 4.4, since $\bar{\Pi}(g(t)) \sim \bar{\Pi}\left(g_{y}^{h}(t-h)\right)$ as $t \rightarrow \infty$,

$$
\begin{aligned}
\mathbb{Q}\left(X_{h} \in d y ; \mathcal{B}_{h}\right) & \stackrel{(3.15)}{=} \mathbb{P}\left(X_{h} \in d y ; \mathcal{B}_{h}\right) \lim _{t \rightarrow \infty} \frac{\mathbb{P}\left(\mathcal{O}_{t-h}^{g_{y}^{h}}\right)}{\mathbb{P}\left(\mathcal{O}_{t}\right)} \\
& \stackrel{4.4}{=} \mathbb{P}\left(X_{h} \in d y ; \mathcal{B}_{h}\right) \lim _{t \rightarrow \infty} \frac{\bar{\Pi}\left(g_{y}^{h}(t-h)\right) \Phi_{y}^{h}(t-h)}{\bar{\Pi}(g(t)) \Phi(t)} \\
& =\mathbb{P}\left(X_{h} \in d y ; \mathcal{B}_{h}\right) \frac{\Phi_{y}^{h}(\infty)}{\Phi(\infty)} .
\end{aligned}
$$

Now we show $\mathbb{Q}\left(X_{h}<\infty\right)=\mathbb{P}(\mathfrak{C}>h)$. Applying (4.6) with $\mathcal{B}_{h}=\mathcal{O}_{h}$,

$$
\begin{aligned}
\mathbb{Q}\left(X_{h}<\infty\right) & =\int_{g(h)}^{\infty} \mathbb{Q}\left(X_{h} \in d y\right)=\int_{g(h)}^{\infty} \frac{\Phi_{y}^{h}(\infty)}{\Phi(\infty)} \mathbb{P}\left(X_{h} \in d y ; \mathcal{O}_{h}\right) \\
& =\frac{1}{\Phi(\infty)} \int_{g(h)}^{\infty} \int_{0}^{\infty} \mathbb{P}\left(\mathcal{O}_{v}^{g_{y}^{h}}\right) d v \mathbb{P}\left(X_{h} \in d y ; \mathcal{O}_{h}\right) \\
& =\frac{1}{\Phi(\infty)} \int_{0}^{\infty} \int_{g(h)}^{\infty} \mathbb{P}\left(\mathcal{O}_{v}^{g_{y}^{h}}\right) \mathbb{P}\left(X_{h} \in d y ; \mathcal{O}_{h}\right) d v .
\end{aligned}
$$

Now, $\mathbb{P}\left(\mathcal{O}_{v}^{g_{y}^{h}}\right) \mathbb{P}\left(X_{h} \in d y ; \mathcal{O}_{h}\right)=\mathbb{P}\left(\mathcal{O}_{v+h} ; X_{h} \in d y\right)$ by (3.11). Then by the definition (3.4) of $\mathcal{O}_{v+h}$,

$$
\begin{aligned}
\mathbb{Q}\left(X_{h}<\infty\right) & =\frac{1}{\Phi(\infty)} \int_{0}^{\infty} \int_{g(h)}^{\infty} \mathbb{P}\left(\mathcal{O}_{v+h} ; X_{h} \in d y\right) d v \\
& =\frac{1}{\Phi(\infty)} \int_{0}^{\infty} \mathbb{P}\left(\mathcal{O}_{v+h} ; X_{h}>g(h)\right) d v \\
& \stackrel{(3.4)}{=} \frac{1}{\Phi(\infty)} \int_{0}^{\infty} \mathbb{P}\left(\mathcal{O}_{v+h}\right) d v
\end{aligned}
$$




$$
=\frac{1}{\Phi(\infty)} \int_{h}^{\infty} \mathbb{P}\left(\mathcal{O}_{u}\right) d u=: \mathbb{P}(\mathfrak{C}>h)
$$

\subsubsection{Proof of Proposition 3.10.}

Proof of Proposition 3.10: For $y>g(x), t>b>a>x>0$, with $a, b, x, y$ fixed, and an event $\mathcal{B}_{X} \in \mathcal{F}_{x}$, where $\left(\mathcal{F}_{u}\right)_{u \geq 0}$ is the natural filtration of $X$, such that $\mathcal{B}_{X} \subseteq \mathcal{O}_{x}$, consider

$$
\lim _{t \rightarrow \infty} \mathbb{P}\left(X_{x} \in d y ; \mathcal{B}_{X} ; \Delta_{1}^{g(t)} \in(a, b) \mid \mathcal{O}_{t}\right) .
$$

If $\Delta_{1}^{g(t)} \in d s$, then $X_{s}>g(t)$, so $\mathcal{O}_{t}$ is fully attained by time $s$, and $\mathcal{O}_{t}$ can be replaced by $\mathcal{O}_{s}$, so

$$
\begin{aligned}
(4.8) & =\lim _{t \rightarrow \infty} \frac{1}{\mathbb{P}\left(\mathcal{O}_{t}\right)} \int_{a}^{b} \mathbb{P}\left(X_{x} \in d y ; \mathcal{B}_{X} ; \Delta_{1}^{g(t)} \in d s ; \mathcal{O}_{t}\right) \\
& =\lim _{t \rightarrow \infty} \frac{1}{\mathbb{P}\left(\mathcal{O}_{t}\right)} \int_{a}^{b} \mathbb{P}\left(X_{x} \in d y ; \mathcal{B}_{X} ; \Delta_{1}^{g(t)} \in d s ; \mathcal{O}_{s}\right) .
\end{aligned}
$$

Recall the definition (3.6). Given $\Delta_{1}^{g(t)}>a>x$, we can replace $\left\{X_{x} \in d y\right\}, \mathcal{B}_{X}$, $\mathcal{O}_{s}$ by corresponding events $\left\{X_{x}^{(0, g(t))} \in d y\right\}, \mathcal{B}_{X^{(0, g(t))}}, \mathcal{O}_{s, X^{(0, g(t))}}$ for the process $X^{(0, g(t))}$ with Lévy measure restricted to $(0, g(t))$, i.e. all jumps larger than $g(t)$ are removed. These events are each independent of $\Delta_{1}^{g(t)}$, and since $\Delta_{1}^{g(t)}$ is exponentially distributed with parameter $\bar{\Pi}(g(t))$,

$$
\begin{aligned}
(4.8) & =\lim _{t \rightarrow \infty} \frac{1}{\mathbb{P}\left(\mathcal{O}_{t}\right)} \int_{a}^{b} \mathbb{P}\left(X_{x}^{(0, g(t))} \in d y ; \mathcal{B}_{X^{(0, g(t))}} ; \Delta_{1}^{g(t)} \in d s ; \mathcal{O}_{s, X^{(0, g(t))}}\right) \\
& =\lim _{t \rightarrow \infty} \frac{1}{\mathbb{P}\left(\mathcal{O}_{t}\right)} \int_{a}^{b} \mathbb{P}\left(X_{x}^{(0, g(t))} \in d y ; \mathcal{B}_{X^{(0, g(t))}} ; \mathcal{O}_{s, X^{(0, g(t))}}\right) \mathbb{P}\left(\Delta_{1}^{g(t)} \in d s\right) \\
& =\lim _{t \rightarrow \infty} \frac{\bar{\Pi}(g(t))}{\mathbb{P}\left(\mathcal{O}_{t}\right)} \int_{a}^{b} \mathbb{P}\left(X_{x}^{(0, g(t))} \in d y ; \mathcal{B}_{X^{(0, g(t))}} ; \mathcal{O}_{s, X^{(0, g(t))}}\right) e^{-\bar{\Pi}(g(t)) s} d s .
\end{aligned}
$$

Now, since $\lim _{t \rightarrow \infty} e^{-\bar{\Pi}(g(t)) s}=1$, uniformly among $s \in(a, b)$,

$$
(4.8)=\lim _{t \rightarrow \infty} \frac{\bar{\Pi}(g(t))}{\mathbb{P}\left(\mathcal{O}_{t}\right)} \int_{a}^{b} \mathbb{P}\left(X_{x}^{(0, g(t))} \in d y ; \mathcal{B}_{X^{(0, g(t))}} ; \mathcal{O}_{s, X^{(0, g(t))}}\right) d s .
$$

Applying Corollary 4.4, and recalling from (4.5) that $\Phi(\infty)<\infty$ when $I(f)<\infty$,

$$
\begin{aligned}
(4.8) & =\frac{1}{\Phi(\infty)} \lim _{t \rightarrow \infty} \int_{a}^{b} \mathbb{P}\left(X_{x}^{(0, g(t))} \in d y ; \mathcal{B}_{X^{(0, g(t))}} ; \mathcal{O}_{s, X^{(0, g(t))}}\right) d s \\
& =\frac{1}{\Phi(\infty)} \lim _{t \rightarrow \infty} \int_{a}^{b} \mathbb{P}\left(X_{x}^{(0, g(t))} \in d y ; \mathcal{B}_{X^{(0, g(t))}} \mid \mathcal{O}_{s, X^{(0, g(t))}}\right) \mathbb{P}\left(\mathcal{O}_{s, X^{(0, g(t))}}\right) d s .
\end{aligned}
$$

Now, $\lim _{t \rightarrow \infty} \mathbb{P}\left(\mathcal{O}_{s, X^{(0, g(t))}}\right) / \mathbb{P}\left(\mathcal{O}_{s}\right)=1$, uniformly among $s \in(a, b)$, so

$$
(4.8)=\lim _{t \rightarrow \infty} \int_{a}^{b} \mathbb{P}\left(X_{x}^{(0, g(t))} \in d y ; \mathcal{B}_{X^{(0, g(t))}} \mid \mathcal{O}_{s, X^{(0, g(t))}}\right) \frac{\mathbb{P}\left(\mathcal{O}_{s}\right)}{\Phi(\infty)} d s,
$$


and similarly $\mathbb{P}\left(X_{x}^{(0, g(t))} \in d y ; \mathcal{B}_{X^{(0, g(t))}} \mid \mathcal{O}_{s, X^{(0, g(t))}}\right) \sim \mathbb{P}\left(X_{x} \in d y ; \mathcal{B}_{X} \mid \mathcal{O}_{s}\right)$ as $t \rightarrow$ $\infty$, uniformly among $s \in(a, b)$. Then by the definition of $\mathfrak{C}$ in (3.21),

$$
\begin{aligned}
(4.8) & =\int_{a}^{b} \mathbb{P}\left(X_{x} \in d y ; \mathcal{B}_{X} \mid \mathcal{O}_{s}\right) \frac{\mathbb{P}\left(\mathcal{O}_{s}\right)}{\Phi(\infty)} d s=\int_{a}^{b} \mathbb{P}\left(X_{x} \in d y ; \mathcal{B}_{X} \mid \mathcal{O}_{s}\right) \mathbb{P}(\mathfrak{C} \in d s) \\
& =: \mathbb{Q}^{\prime}\left(X_{x} \in d y ; \mathcal{B}_{X} ; T_{\infty}^{\prime} \in(a, b)\right) .
\end{aligned}
$$

It is clear that (4.11) uniquely determines the limit measure $\mathbb{Q}^{\prime}(\cdot)$ on $\mathcal{D}[0, \infty) \times$ $(0, \infty)$. To verify that $T_{\infty}^{\prime}$ under $\mathbb{Q}^{\prime}(\cdot)$ has the desired properties, by (4.11) with $\mathcal{B}_{X}=\mathcal{O}_{x}$, since $x<a<s$,

$$
\begin{aligned}
\mathbb{Q}^{\prime}\left(T_{\infty}^{\prime} \in(a, b)\right) & =\int_{g(x)}^{\infty} \mathbb{Q}^{\prime}\left(X_{x} \in d y ; \mathcal{O}_{x} ; T_{\infty}^{\prime} \in(a, b)\right) \\
& =\int_{g(x)}^{\infty} \int_{a}^{b} \mathbb{P}\left(X_{x} \in d y ; \mathcal{O}_{x} \mid \mathcal{O}_{s}\right) \mathbb{P}(\mathfrak{C} \in d s) \\
& =\int_{a}^{b} \mathbb{P}\left(X_{x}>g(x) ; \mathcal{O}_{x} \mid \mathcal{O}_{s}\right) \mathbb{P}(\mathfrak{C} \in d s) \\
& =\int_{a}^{b} \mathbb{P}(\mathfrak{C} \in d s)=\mathbb{P}(\mathfrak{C} \in(a, b))=\mathbb{Q}\left(T_{\infty} \in(a, b)\right) .
\end{aligned}
$$

Similarly, by (4.11) with $\mathcal{B}_{X}=\mathcal{O}_{x}$, taking limits as $a \rightarrow x$ and $b \rightarrow \infty$, since $x<s$, we also have

$$
\begin{aligned}
\mathbb{Q}^{\prime}\left(X_{x}<\infty\right) & =\int_{g(x)}^{\infty} \int_{x}^{\infty} \mathbb{P}\left(X_{x} \in d y ; \mathcal{O}_{x} \mid \mathcal{O}_{s}\right) \mathbb{P}(\mathfrak{C} \in d s) \\
& =\int_{x}^{\infty} \mathbb{P}\left(X_{x}>g(x) ; \mathcal{O}_{x} \mid \mathcal{O}_{s}\right) \mathbb{P}(\mathfrak{C} \in d s) \\
& =\int_{x}^{\infty} \mathbb{P}(\mathfrak{C} \in d s)=\mathbb{P}(\mathfrak{C}>x)=\mathbb{Q}^{\prime}\left(T_{\infty}^{\prime}>x\right),
\end{aligned}
$$

so that $T_{\infty}^{\prime}$ is indeed the explosion time for the process $X$ under $\mathbb{Q}^{\prime}(\cdot)$.

\subsubsection{Proof of Proposition 3.11.}

Proof of Proposition 3.11: Recall $\Delta_{1}^{g(t)}$ is the time of $X$ 's first jump bigger than $g(t), \pi_{h}(X)$ is the sample path of $X$ up to time $h, F_{1}$ is a functional on the excursion process, and $\mathcal{B} \subseteq \mathcal{O}_{h}, \mathcal{B} \in \mathcal{F}_{h}$, where $\left(\mathcal{F}_{u}\right)_{u \geq 0}$ is $X$ 's natural filtration. For fixed $b>a>h>0$, disintegrating on the values of $\Delta_{1}^{g(t)}$ and $\pi_{h}(X)$,

$$
\begin{gathered}
\mathbb{E}\left[\mathbb{1}_{\left\{\pi_{h}(X) \in \mathcal{B}\right\}} \mathbb{1}_{\left\{\Delta_{1}^{g(t)} \in(a, b)\right\}} F_{1}\left(\pi_{h}\left(\left(\varepsilon_{s}\right)_{s \geq 0}\right)\right) \mid \mathcal{O}_{t}\right] \\
=\int_{\nu \in \mathcal{B}} \int_{u \in(a, b)} \mathbb{E}\left[F_{1}\left(\pi_{h}\left(\left(\varepsilon_{s}\right)_{s \geq 0}\right)\right) \mid \mathcal{O}_{t} ; \pi_{h}(X)=\nu ; \Delta_{1}^{g(t)}=u\right] \\
\times \mathbb{P}\left(\pi_{h}(X) \in d \nu ; \Delta_{1}^{g(t)} \in d u \mid \mathcal{O}_{t}\right) .
\end{gathered}
$$

Given a fixed path $\pi_{h}(X)=\nu, \pi_{h}\left(\left(\varepsilon_{s}\right)_{s \geq 0}\right)$ depends only on $\nu$, so $\pi_{h}\left(\left(\varepsilon_{s}\right)_{s \geq 0}\right)$ is conditionally independent of $\Delta_{1}^{g(t)}$ and $\mathcal{O}_{t}$. Here, $h<a<u$, so the excursion process $\left(\varepsilon_{s}\right)_{s \geq 0}$ contains only excursions of length at most $g(t)$, so we may replace 
$\pi_{h}\left(\left(\varepsilon_{s}\right)_{s \geq 0}\right)$ by $\pi_{h}\left(\left(\varepsilon_{s}^{g(t)}\right)_{s \geq 0}\right)$, where $\left(\varepsilon_{s}^{g(t)}\right)_{s \geq 0}$ is the excursion process sampled using the conditional excursion measure on the space of excursions of length at most $g(t)$, so

$$
(4.12)=\int_{\mathcal{B}} \int_{a}^{b} \mathbb{E}\left[F_{1}\left(\pi_{h}\left(\left(\varepsilon_{s}^{g(t)}\right)_{s \geq 0}\right)\right) \mid \pi_{h}(X)=\nu\right] \mathbb{P}\left(\pi_{h}(X) \in d \nu ; \Delta_{1}^{g(t)} \in d u \mid \mathcal{O}_{t}\right),
$$

Now, $\lim _{t \rightarrow \infty} \mathbb{E}\left[F_{1}\left(\pi_{h}\left(\left(\varepsilon_{s}^{g(t)}\right)_{s \geq 0}\right)\right) \mid \pi_{h}(X)=\nu\right]=\mathbb{E}\left[F_{1}\left(\pi_{h}\left(\left(\varepsilon_{s}\right)_{s \geq 0}\right)\right) \mid \pi_{h}(X)=\right.$ $\nu]$, and moreover by Proposition 3.10, $\lim _{t \rightarrow \infty} \mathbb{P}\left(\pi_{h}(X) \in d \nu ; \Delta_{1}^{g(t)} \in d u \mid \mathcal{O}_{t}\right)=$ $\mathbb{Q}^{\prime}\left(\pi_{h}(X) \in d \nu ; T_{\infty}^{\prime} \in d u\right)$, so

$$
\begin{aligned}
\lim _{t \rightarrow \infty}(4.12) & =\int_{\mathcal{B}} \int_{a}^{b} \mathbb{E}\left[F_{1}\left(\pi_{h}\left(\left(\varepsilon_{s}\right)_{s \geq 0}\right)\right) \mid \pi_{h}(X)=\nu\right] \mathbb{Q}^{\prime}\left(\pi_{h}(X) \in d \nu ; T_{\infty}^{\prime} \in d u\right) \\
& =: \mathbb{E}_{\mathbb{Q}^{\prime \prime}}\left[F_{1}\left(\pi_{h}\left(\left(\varepsilon_{s}\right)_{s \geq 0}\right)\right) \mathbb{1}_{\left\{\pi_{h}(X) \in \mathcal{B}\right\}} \mathbb{1}_{\left\{T_{\infty}^{\prime \prime} \in(a, b)\right\}},\right.
\end{aligned}
$$

where we are able to exchange the order of limits and integration since $F_{1}$ is bounded. Taking $F_{1} \equiv 1$, it follows immediately that $\mathbb{Q}^{\prime \prime}(\cdot)$ and $\mathbb{Q}^{\prime}(\cdot)$ agree on $\mathcal{D}[0, \infty) \times(0, \infty)$. The weak convergence of $\left(\left(M_{t}\right)_{X_{\Delta-}>t \geq 0},\left(X_{s}\right)_{\Delta>s \geq 0}, \Delta\right)$ under

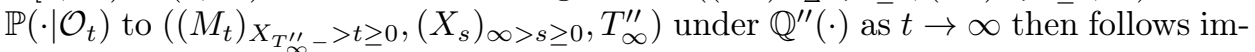
mediately from the fact (see e.g. Bertoin, 1996, Ex. IV.6.3 or Kolb and Savov, 2016, p4113) that for all $x>0,\left(M_{t}\right)_{X_{x-}>t \geq 0}$ is uniquely determined by $\left(\varepsilon_{s}\right)_{x>s \geq 0}$ and $\left(X_{s}\right)_{x>s \geq 0}$, and both of $\left(\varepsilon_{s}\right)_{x>s \geq 0}$ and $\left(X_{s}\right)_{x>s \geq 0}$ have weak limits as determined in (4.13). That is, we construct $M$ pathwise via its excursions using $\left(X_{u}\right)_{\infty>u \geq 0}$ to determine the timing and length of each excursion, where we sample the excursions of $M$ until time $X_{s-}$ using the excursion measure conditional on each excursion length. Similarly, the explicit description of the behaviour of $M$ until time $X_{T_{\infty}^{\prime \prime}-}$ under $\mathbb{Q}^{\prime \prime}(\cdot)$ follows immediately from the definition of $\mathbb{Q}^{\prime}(\cdot)$ in (3.22), using the fact that $\mathbb{Q}^{\prime \prime}(\cdot)$ and $\mathbb{Q}^{\prime}(\cdot)$ agree on $\mathcal{D}[0, \infty) \times(0, \infty)$.

\subsubsection{Proof of Theorem 3.12.}

Proof of Theorem 3.12: As $X$ determines the lengths and timings of excursions of $M$ (see Bertoin, 1996, Ex. IV.6.3), it follows that for all $K>0$ and $b>a>0$, for all $t$ large enough that $g(t)>K$,

$$
\left\{\Delta_{1}^{g(t)} \in(a, b)\right\}=\left\{\Delta_{1}^{g(t)} \in(a, b)\right\} \cap\left\{M_{v} \neq 0 \text {, for all } v \in\left(X_{\Delta_{1}^{g(t)}-}, X_{\Delta_{1}^{g(t)}-}+K\right)\right\} .
$$

Let us assume that a weak limit measure $\hat{\mathbb{Q}}(\cdot)=\lim _{t \rightarrow \infty} \mathbb{P}\left(\cdot \mid \mathcal{O}_{t}\right)$ exists on the space containing $\left(M_{t}\right)_{t \geq 0}$. Such a measure must agree with $\mathbb{Q}^{\prime}(\cdot)$ on $\mathcal{D}[0, \infty) \times(0, \infty)$, as we proved in Proposition 3.10 that any such limit measure is uniquely determined on $\mathcal{D}[0, \infty) \times(0, \infty)$ by (3.22). It follows that for all $K>0$ and $b>a>0$,

$$
\begin{gathered}
\lim _{t \rightarrow \infty} \mathbb{P}\left(M_{v} \neq 0, \text { for all } v \in\left(X_{\Delta_{1}^{g(t)}-}, X_{\Delta_{1}^{g(t)}-}+K\right) ; \Delta_{1}^{g(t)} \in(a, b) \mid \mathcal{O}_{t}\right) \\
=\hat{\mathbb{Q}}\left(M_{v} \neq 0, \text { for all } v \in\left(X_{\hat{T}_{\infty}-}, X_{\hat{T}_{\infty}-}+K\right) ; \hat{T}_{\infty} \in(a, b)\right),
\end{gathered}
$$

where $\hat{T}_{\infty}$ is the explosion time for $X$ under $\hat{\mathbb{Q}}(\cdot)$. But also by (4.14) and uniqueness of the limit measure on $\mathcal{D}[0, \infty) \times(0, \infty)$, we have for all $K>0$ and $b>a>0$,

$$
\hat{\mathbb{Q}}\left(M_{v} \neq 0, \text { for all } v \in\left(X_{\hat{T}_{\infty}-}, X_{\hat{T}_{\infty}-}+K\right) ; \hat{T}_{\infty} \in(a, b)\right)=(4.15)
$$




$$
=\lim _{t \rightarrow \infty} \mathbb{P}\left(\Delta_{1}^{g(t)} \in(a, b) \mid \mathcal{O}_{t}\right)=\hat{\mathbb{Q}}\left(\hat{T}_{\infty} \in(a, b)\right),
$$

from which it follows immediately that $M$ is transient under $\hat{\mathbb{Q}}(\cdot)$, as required.

4.2. Proofs in the $I(f)=\infty$ Case. The next three proofs require Lemma 4.6, proven in Section 7.

Lemma 4.6. In case (i), for $t_{0}(y)$ as in (3.20), uniformly in $h>0, y>g(h)$, and $t \in\left(t_{0}(y), \infty\right]$,

$$
\int_{t_{0}(y)}^{t}(\bar{\Pi}(g(s+h)-y)-\bar{\Pi}(g(s))) d s \lesssim y f^{\prime}(y) \bar{\Pi}(y) .
$$

\subsubsection{Proof of Theorem 3.14.}

Proof of Theorem 3.14: For fixed $h>0, y>g(h)$, we will prove that $q_{h}(y):=$ $\lim _{t \rightarrow \infty} \mathbb{P}\left(\mathcal{O}_{t-h}^{g_{y}^{h}}\right) / \mathbb{P}\left(\mathcal{O}_{t}\right)<\infty$. For each $h>0, y>g(h)$, note that $g(t) \sim g_{y}^{h}(t-h)$ by the properties of $g(t)$ given in Assumption 3.1. Hence $\bar{\Pi}(g(t)) \sim \bar{\Pi}\left(g_{y}^{h}(t-h)\right)$ as $t \rightarrow \infty$, since $\bar{\Pi}$ is CRV at $\infty$. Thus, applying Corollary 4.4,

$$
\lim _{t \rightarrow \infty} \frac{\mathbb{P}\left(\mathcal{O}_{t-h}^{g_{y}^{h}}\right)}{\mathbb{P}\left(\mathcal{O}_{t}\right)}=\lim _{t \rightarrow \infty} \frac{\bar{\Pi}\left(g_{y}^{h}(t-h)\right) \Phi_{y}^{h}(t)}{\bar{\Pi}(g(t)) \Phi(t)}=\lim _{t \rightarrow \infty} \frac{\Phi_{y}^{h}(t)}{\Phi(t)} .
$$

Then by (3.18) and (3.19), for $t_{0}(y)$ as defined in (3.20),

$$
(4.17)=\frac{\Phi_{y}^{h}\left(t_{0}(y)\right)}{\Phi(1)} \lim _{t \rightarrow \infty} \exp \left(\int_{t_{0}(y)}^{t}\left(\bar{\Pi}\left(g_{y}^{h}(s)\right)+\rho_{y}^{h}(s)\right) d s-\int_{1}^{t}(\bar{\Pi}(g(s))+\rho(s)) d s\right) .
$$

By (4.1) in Lemma 4.2, the integral $\int_{t_{0}(y)}^{\infty} \rho_{y}^{h}(s) d s$ is uniformly bounded for all $h>0$, $y>g(h)$. By Lemma 4.3, it follows that $-\int_{1}^{\infty} \rho(s) d s<\infty$, so

$$
\begin{aligned}
(4.17) & \lesssim \frac{\Phi_{y}^{h}\left(t_{0}(y)\right)}{\Phi(1)} \lim _{t \rightarrow \infty} \exp \left(\int_{t_{0}(y)}^{t} \bar{\Pi}\left(g_{y}^{h}(s)\right) d s-\int_{1}^{t} \bar{\Pi}(g(s)) d s\right) \\
& \lesssim \frac{\Phi_{y}^{h}\left(t_{0}(y)\right)}{\Phi(1)} \lim _{t \rightarrow \infty} \exp \left(\int_{t_{0}(y)}^{t}\left(\bar{\Pi}\left(g_{y}^{h}(s)\right)-\bar{\Pi}(g(s))\right) d s\right) .
\end{aligned}
$$

Applying Lemma 4.6, and recalling that $y f^{\prime}(y) \bar{\Pi}(y)$ decreases to zero as $y \rightarrow \infty$,

$$
(4.17) \lesssim \frac{\Phi_{y}^{h}\left(t_{0}(y)\right)}{\Phi(1)} \exp \left(y f^{\prime}(y) \bar{\Pi}(y)\right)<\infty .
$$

Now, $q_{h}(y):=\lim _{t \rightarrow \infty} \mathbb{P}\left(\mathcal{O}_{t-h}^{g_{y}^{h}}\right) / \mathbb{P}\left(\mathcal{O}_{t}\right)$ is non-decreasing in $y$ since for all $y<y^{\prime}$, $g_{y}^{h}(t)=g(t+h)-y>g(t+h)-y^{\prime}=g_{y^{\prime}}^{h}(t)$, and so $\mathbb{P}\left(\mathcal{O}_{t-h}^{g_{y}^{h}}\right) \leq \mathbb{P}\left(\mathcal{O}_{t-h}^{g_{y^{\prime}}^{h}}\right)$. Finally, we conclude by (3.15) that $\mathbb{Q}\left(X_{h} \in d y ; \mathcal{B}_{h}\right)=\mathbb{P}\left(X_{h} \in d y ; \mathcal{B}_{h}\right) q_{h}(y)$, as required. 
Proof of Proposition 3.15: For $h>0, \lim _{t \rightarrow \infty} \mathbb{P}\left(X_{h} \in(g(h), \infty) \mid \mathcal{O}_{t}\right)=1$. We will prove by dominated convergence Kallenberg (2002, Theorem 1.21) that limits and integration can be exchanged from (4.18) to (4.19), so by (3.15), for all $h>0$,

$$
\begin{aligned}
1=\lim _{t \rightarrow \infty} \mathbb{P}\left(X_{h} \in(g(h), \infty) \mid \mathcal{O}_{t}\right) & =\lim _{t \rightarrow \infty} \int_{g(h)}^{\infty} \mathbb{P}\left(X_{h} \in d y \mid \mathcal{O}_{t}\right) \\
& =\lim _{t \rightarrow \infty} \int_{g(h)}^{\infty} \frac{\mathbb{P}\left(\mathcal{O}_{t-h}^{g_{y}^{h}}\right)}{\mathbb{P}\left(\mathcal{O}_{t}\right)} \mathbb{P}\left(X_{h} \in d y ; \mathcal{O}_{h}\right) \\
& =\int_{g(h)}^{\infty} \lim _{t \rightarrow \infty} \frac{\mathbb{P}\left(\mathcal{O}_{t-h}^{g_{y}^{h}}\right)}{\mathbb{P}\left(\mathcal{O}_{t}\right)} \mathbb{P}\left(X_{h} \in d y ; \mathcal{O}_{h}\right) \\
& =\mathbb{Q}\left(X_{h} \in(g(h), \infty)\right),
\end{aligned}
$$

as required. For $A>3 \vee(B-1)$, we will bound the integral over $(g(h), \infty)$ via:

$$
\left[\frac{g(t-h)}{A}, \infty\right) \cup(g(h), g(h+1)] \cup\left(g(h+1), \frac{g(t-h)}{A}\right)=: I_{1} \cup I_{2} \cup I_{3} .
$$

Proof for $I_{1}$. Since $y \in I_{1}$ if and only if $t \leq f(A y)+h$, by Corollary 4.4,

$$
\begin{aligned}
\int_{g(h)}^{\infty} \mathbb{1}_{\left\{y \in I_{1}\right\}} \frac{\mathbb{P}\left(\mathcal{O}_{t-h}^{g_{y}^{h}}\right)}{\mathbb{P}\left(\mathcal{O}_{t}\right)} \mathbb{P}\left(X_{h} \in d y ; \mathcal{O}_{h}\right) & \lesssim \int_{g(h)}^{\infty} \frac{\mathbb{1}_{\left\{y \in I_{1}\right\}}}{\bar{\Pi}(g(t)) \Phi(t)} \mathbb{P}\left(X_{h} \in d y\right) \\
& =\frac{\mathbb{P}\left(X_{h} \geq \frac{g(t-h)}{A}\right)}{\bar{\Pi}(g(t)) \Phi(t)} .
\end{aligned}
$$

Now, $I(f)=\infty$, so $\lim _{t \rightarrow \infty} \Phi(t)=\infty$ by (4.5), and it suffices to show that

$$
\limsup _{t \rightarrow \infty} \frac{\mathbb{P}\left(X_{h} \geq \frac{g(t-h)}{A}\right)}{\bar{\Pi}(g(t))}
$$

is finite for each fixed $h>0$, as the integal in (4.18) over the region $I_{1}$ tends to 0 as $t \rightarrow \infty$, so the dominated convergence theorem applies, trivially, on $I_{1}$.

Recall the notation in (3.7). Observe that $\Delta_{1}^{g(t)}$ has exponential distribution of rate $\bar{\Pi}(g(t))$, so

$$
\begin{aligned}
\mathbb{P}\left(X_{h} \geq \frac{g(t-h)}{A}\right) & =\mathbb{P}\left(X_{h} \geq \frac{g(t-h)}{A} ; \Delta_{1}^{g(t)}>h\right)+\mathbb{P}\left(X_{h} \geq \frac{g(t-h)}{A} ; \Delta_{1}^{g(t)} \leq h\right) \\
& \leq \mathbb{P}\left(X_{h}^{(0, g(t))} \geq \frac{g(t-h)}{A}\right)+\mathbb{P}\left(\Delta_{1}^{g(t)} \leq h\right) \\
& =\mathbb{P}\left(X_{h}^{(0, g(t))} \geq \frac{g(t-h)}{A}\right)+1-e^{-h \bar{\Pi}(g(t))} \\
& \leq \mathbb{P}\left(X_{h}^{(0, g(t))} \geq \frac{g(t-h)}{A}\right)+h \bar{\Pi}(g(t)),
\end{aligned}
$$

where $X^{(0, g(t))}$ has the same Lévy measure as $X$, but restricted to $(0, g(t))$, so $X^{(0, g(t))}$ has no jumps larger than $g(t)$. By (4.23) and Markov's inequality,

$$
(4.22)-h \leq \limsup _{t \rightarrow \infty} \frac{\mathbb{P}\left(X_{h}^{(0, g(t))} \geq \frac{g(t-h)}{A}\right)}{\bar{\Pi}(g(t))}
$$




$$
\lesssim \limsup _{t \rightarrow \infty} \frac{A \mathbb{E}\left[X_{h}^{(0, g(t))}\right]}{\bar{\Pi}(g(t)) g(t-h)}=\limsup _{t \rightarrow \infty} \frac{A h \int_{0}^{g(t)} x \Pi(d x)}{\bar{\Pi}(g(t)) g(t-h)} .
$$

Now, $\int_{0}^{g(t)} x \Pi(d x)=\int_{x=0}^{g(t)} \int_{y=0}^{x} d y \Pi(d x)=\int_{y=0}^{g(t)} \int_{x=y}^{g(t)} \Pi(d x) d y \leq \int_{0}^{g(t)} \bar{\Pi}(y) d y$. Then because $\bar{\Pi}$ is regularly varying at $\infty$, by Karamata's theorem (Bingham et al., 1989, Prop 1.5.8), we deduce, as required for dominated convergence on $I_{1}$, that for each $h>0$,

$$
\begin{aligned}
(4.22) & \lesssim h+\limsup _{t \rightarrow \infty} \frac{A h \int_{0}^{1} \bar{\Pi}(y) d y+A h \int_{1}^{g(t)} \bar{\Pi}(y) d y}{\bar{\Pi}(g(t)) g(t-h)} \\
& \lesssim h+\limsup _{t \rightarrow \infty} \frac{g(t) \bar{\Pi}(g(t))}{\bar{\Pi}(g(t)) g(t-h)}<\infty .
\end{aligned}
$$

Proof for $I_{2}$. By Theorem 3.14, $q_{h}(y)$ is non-decreasing in $y$, so by $(3.23), \mathbb{P}\left(\mathcal{O}_{t-h}^{g_{y}^{h}}\right)$ is non-decreasing in $y$. Now, $\lim _{t \rightarrow \infty} \mathbb{P}\left(\mathcal{O}_{t-h}^{g_{g(h+1)}^{h}}\right) / \mathbb{P}\left(\mathcal{O}_{t}\right)=q_{h}(g(h+1))<\infty$ for each fixed $h$ by Theorem 3.14, and we conclude that

$$
\begin{aligned}
& \lim _{t \rightarrow \infty} \int_{g(h)}^{\infty} \mathbb{1}_{\left\{y \in I_{2}\right\}} \frac{\mathbb{P}\left(\mathcal{O}_{t-h}^{g_{y}^{h}}\right)}{\mathbb{P}\left(\mathcal{O}_{t}\right)} \mathbb{P}\left(X_{h} \in d y ; \mathcal{O}_{h}\right) \\
& \leq \lim _{t \rightarrow \infty} \frac{\mathbb{P}\left(\mathcal{O}_{t-h}^{g_{g(h+1)}^{h}}\right)}{\mathbb{P}\left(\mathcal{O}_{t}\right)} \int_{g(h)}^{\infty} \mathbb{1}_{\left\{y \in I_{2}\right\}} \mathbb{P}\left(X_{h} \in d y ; \mathcal{O}_{h}\right) \\
& =q_{h}(g(h+1)) \mathbb{P}\left(X_{h} \in I_{2} ; \mathcal{O}_{h}\right),
\end{aligned}
$$

which is finite for each $h>0$, so dominated convergence applies on $I_{2}$.

Proof for $I_{3}$. By (3.17) and Corollary 4.4, for all large enough $t$,

$$
\frac{\mathbb{P}\left(\mathcal{O}_{t-h}^{g_{y}^{h}}\right)}{\mathbb{P}\left(\mathcal{O}_{t}\right)} \leq 2 \frac{\left[\bar{\Pi}\left(g_{y}^{h}(t-h)\right)+\rho_{y}^{h}(t-h)\right] \Phi_{y}^{h}(t-h)}{\bar{\Pi}(g(t)) \Phi(t)} .
$$

For $y \in I_{3}, y>g(h+1)$, so $f(y)-h>f(g(h+1))-h=1$, and $1+1 /(f(y)-h)<2$. By (4.2), as $\lim _{t \rightarrow \infty} u(t)=0$, for all large enough $t$ and for all $y \in I_{3}$,

$$
\begin{aligned}
& \frac{\mathbb{P}\left(\mathcal{O}_{t-h}^{g_{y}^{h}}\right)}{\mathbb{P}\left(\mathcal{O}_{t}\right)} \leq 2 \frac{\left(1+u(t)\left(1+\frac{1}{f(y)-h}\right)\right) \bar{\Pi}\left(g_{y}^{h}(t-h)\right) \Phi_{y}^{h}(t-h)}{\bar{\Pi}(g(t)) \Phi(t)} \\
& \frac{\mathbb{P}\left(\mathcal{O}_{t-h}^{g_{y}^{h}}\right)}{\mathbb{P}\left(\mathcal{O}_{t}\right)} \leq 6 \frac{\bar{\Pi}\left(g_{y}^{h}(t-h)\right) \Phi_{y}^{h}(t-h)}{\bar{\Pi}(g(t)) \Phi(t)} .
\end{aligned}
$$

Now, $y<g(t-h) / A<g(t) / 3$ for $y \in I_{3}$, so by (3.10), since $\bar{\Pi}$ is regularly varying at $\infty, \bar{\Pi}\left(g_{y}^{h}(t-h)\right)=\bar{\Pi}(g(t)-y) \lesssim \bar{\Pi}(g(t))$, uniformly among $y \in I_{3}$. So for each fixed $h>0$, for all large enough $t$, uniformly in $y \in I_{3}$,

$$
\frac{\mathbb{P}\left(\mathcal{O}_{t-h}^{g_{y}^{h}}\right)}{\mathbb{P}\left(\mathcal{O}_{t}\right)} \lesssim \frac{\Phi_{y}^{h}(t-h)}{\Phi(t)} .
$$


Now, by (3.19), for $t_{0}(y)$ as defined in (3.20), we have

$$
\Phi_{y}^{h}(t-h)=\Phi_{y}^{h}\left(t_{0}(y)\right) \exp \left(\int_{t_{0}(y)}^{t-h} \bar{\Pi}\left(g_{y}^{h}(s)\right) d s+\int_{t_{0}(y)}^{t-h} \rho_{y}^{h}(s) d s\right)
$$

Applying (4.1) and recalling that $1+1 /(f(y)-h)<2$ for $y \in I_{3}$, the integral $\int_{t_{0}(y)}^{\infty} \rho_{y}^{h}(s) d s$ is uniformly bounded among $y$, so uniformly among $y \in I_{3}$,

$$
\Phi_{y}^{h}(t-h) \lesssim \Phi_{y}^{h}\left(t_{0}(y)\right) \exp \left(\int_{t_{0}(y)}^{t-h} \bar{\Pi}\left(g_{y}^{h}(s)\right) d s\right)
$$

By Lemma 4.3, $\liminf _{t \rightarrow \infty} \int_{1}^{t} \rho(s) d s>-\infty$, so by (3.16),

$$
\begin{aligned}
\Phi(t) & =\Phi(1) \exp \left(\int_{1}^{t} \bar{\Pi}(g(s)) d s+\int_{1}^{t} \rho(s) d s\right) \\
& \gtrsim \exp \left(\int_{1}^{t} \bar{\Pi}(g(s)) d s\right) .
\end{aligned}
$$

Since $y>g(h+1)$ in $I_{3}$, recalling (3.20), $t_{0}(y) \geq f(A y)>f(y) \geq h+1>1$, so

$$
\begin{aligned}
\frac{\Phi_{y}^{h}(t-h)}{\Phi(t)} & \lesssim \Phi_{y}^{h}\left(t_{0}(y)\right) \exp \left(\int_{t_{0}(y)}^{t-h} \bar{\Pi}\left(g_{y}^{h}(s)\right) d s-\int_{1}^{t} \bar{\Pi}(g(s)) d s\right) \\
& \leq \Phi_{y}^{h}\left(t_{0}(y)\right) \exp \left(\int_{t_{0}(y)}^{t}\left(\bar{\Pi}\left(g_{y}^{h}(s)\right)-\bar{\Pi}(g(s))\right) d s\right)
\end{aligned}
$$

Now, by Lemma 4.6, since $y f^{\prime}(y) \bar{\Pi}(y)$ decreases to zero as $y \rightarrow \infty$, we have uniformly among $y>g(h)$,

$$
\begin{aligned}
\int_{t_{0}(y)}^{t}\left(\bar{\Pi}\left(g_{y}^{h}(s)\right)-\bar{\Pi}(g(s))\right) d s & \leq \int_{t_{0}(y)}^{\infty}\left(\bar{\Pi}\left(g_{y}^{h}(s)\right)-\bar{\Pi}(g(s))\right) d s \\
& \lesssim \sup _{y>g(h)} y f^{\prime}(y) \bar{\Pi}(y)<\infty .
\end{aligned}
$$

So for each fixed $h>0$, uniformly among $y>g(h)$, by (3.12),

$$
\lim _{t \rightarrow \infty} \frac{\Phi_{y}^{h}(t-h)}{\Phi(t)} \lesssim \Phi_{y}^{h}\left(t_{0}(y)\right) e^{-\int_{1}^{t_{0}(y)} \bar{\Pi}(g(s)) d s} \leq t_{0}(y) e^{-\int_{1}^{t_{0}(y)} \bar{\Pi}(g(s)) d s} .
$$

Now, it follows from (4.24) and (4.25) that for each fixed $h>0$, uniformly for all $t$ large enough,

$$
\begin{aligned}
& \int_{g(h)}^{\infty} \mathbb{1}_{\left\{y \in I_{3}\right\}} \frac{\mathbb{P}\left(\mathcal{O}_{t-h}^{g_{y}^{h}}\right)}{\mathbb{P}\left(\mathcal{O}_{t}\right)} \mathbb{P}\left(X_{h} \in d y ; \mathcal{O}_{h}\right) \\
& \lesssim \int_{g(h)}^{\infty} \mathbb{1}_{\left\{y \in I_{3}\right\}} t_{0}(y) e^{-\int_{1}^{t_{0}(y)} \bar{\Pi}(g(s)) d s} \mathbb{P}\left(X_{h} \in d y ; \mathcal{O}_{h}\right)
\end{aligned}
$$


Now (by choice of $A$ sufficiently large if necessary) we have $t_{0}(y)=f(A y) \vee(1+$ $2 / A)=f(A y)$ for all $y>g(h+1)>g(1)$. Then writing $\zeta(x):=x e^{-\int_{1}^{x} \bar{\Pi}(g(u)) d u}$, noting $\zeta(\cdot)$ is differentiable and $\zeta(1)=1$,

$$
\begin{aligned}
(4.26) & =\int_{g(h)}^{\infty} \mathbb{1}_{\left\{y \in I_{3}\right\}} f(A y) e^{-\int_{1}^{f(A y)} \bar{\Pi}(g(s)) d s} \mathbb{P}\left(X_{h} \in d y ; \mathcal{O}_{h}\right) \\
& =\int_{g(h+1)}^{\infty} f(A y) e^{-\int_{1}^{f(A y)} \bar{\Pi}(g(s)) d s} \mathbb{P}\left(X_{h} \in d y ; \mathcal{O}_{h}\right) \\
& \leq \int_{g(h+1)}^{\infty} f(A y) e^{-\int_{1}^{f(A y)} \bar{\Pi}(g(s)) d s} \mathbb{P}\left(X_{h} \in d y\right)=\int_{g(h+1)}^{\infty} \zeta(f(A y)) \mathbb{P}\left(X_{h} \in d y\right) \\
& =\int_{g(h+1)}^{\infty}\left(\int_{1}^{f(A y)} \zeta^{\prime}(x) d x+1\right) \mathbb{P}\left(X_{h} \in d y\right) \\
& =\mathbb{P}\left(X_{h} \geq g(h+1)\right)+\int_{g(h+1)}^{\infty} \int_{1}^{f(A y)} \zeta^{\prime}(x) d x \mathbb{P}\left(X_{h} \in d y\right) .
\end{aligned}
$$

Changing the order of integration, and then applying the result that for each fixed $c, h>0, \mathbb{P}\left(X_{h} \geq z\right) \asymp \bar{\Pi}(z)$, uniformly in $z>c$, since $\bar{\Pi}$ is regularly varying, see Embrechts et al. (1979, Theorem 1 (iii)),

$$
\begin{aligned}
\int_{1}^{\infty} \zeta^{\prime}(x) \int_{g(h+1) \vee \frac{g(x)}{A}}^{\infty} \mathbb{P}\left(X_{h} \in d y\right) d x & \leq \int_{1}^{\infty} \zeta^{\prime}(x) \int_{\frac{g(x)}{A}}^{\infty} \mathbb{P}\left(X_{h} \in d y\right) d x \\
& =\int_{1}^{\infty} \zeta^{\prime}(x) \mathbb{P}\left(X_{h} \geq \frac{g(x)}{A}\right) d x \\
& \asymp \int_{1}^{\infty} \zeta^{\prime}(x) \bar{\Pi}\left(\frac{g(x)}{A}\right) d x \\
& \lesssim \int_{1}^{\infty} \zeta^{\prime}(x) \bar{\Pi}(g(x)) d x \\
& =\int_{1}^{\infty}\left[e^{-\int_{1}^{x} \bar{\Pi}(g(u)) d u}-x \bar{\Pi}(g(x)) e^{-\int_{1}^{x} \bar{\Pi}(g(u)) d u}\right] \\
& \times \bar{\Pi}(g(x)) d x \\
& \leq \int_{1}^{\infty} e^{-\int_{1}^{x} \bar{\Pi}(g(u)) d u} \bar{\Pi}(g(x)) d x \\
& =\int_{1}^{\infty} \frac{d}{d x}\left(-e^{-\int_{1}^{x} \bar{\Pi}(g(u)) d u}\right) d x<\infty
\end{aligned}
$$

and therefore $(4.26)<\infty$, so the dominated convergence theorem applies on $I_{3}$, and the order of limits and integration can be swapped between (4.18) and (4.19), as required.

Proof of Proposition 3.16: Recall $\pi_{h}(X)$ is the sample path of $X$ up to time $h, F_{1}$ is a functional on the excursion process, and $\mathcal{B} \subseteq \mathcal{O}_{h}, \mathcal{B} \in \mathcal{F}_{h}$, where $\left(\mathcal{F}_{u}\right)_{u \geq 0}$ is $X$ 's filtration. Disintegrating on the value of $\pi_{h}(X) \in \mathcal{B}$,

$$
\mathbb{E}\left[F_{1}\left(\pi_{h}\left(\left(\varepsilon_{s}\right)_{s \geq 0}\right)\right) \mathbb{1}_{\left\{\pi_{h}(X) \in \mathcal{B}\right\}} \mid \mathcal{O}_{t}\right]
$$




$$
=\int_{\nu \in \mathcal{B}} \mathbb{E}\left[F_{1}\left(\pi_{h}\left(\left(\varepsilon_{s}\right)_{s \geq 0}\right)\right) \mid \mathcal{O}_{t} ; \pi_{h}(X)=\nu\right] \mathbb{P}\left(\pi_{h}(X) \in d \nu \mid \mathcal{O}_{t}\right) .
$$

Given a fixed path $\pi_{h}(X)=\nu, \pi_{h}\left(\left(\varepsilon_{s}\right)_{s \geq 0}\right)$ depends only on $\nu$, so $\pi_{h}\left(\left(\varepsilon_{s}\right)_{s \geq 0}\right)$ is conditionally independent of $\mathcal{O}_{t}$, and then as $\lim _{t \rightarrow \infty} \mathbb{P}\left(\pi_{h}(X) \in d \nu \mid \mathcal{O}_{t}\right)=\mathbb{Q}\left(\pi_{h}(X) \in\right.$ $d \nu$ ) by Theorem 3.14,

$$
\begin{aligned}
\lim _{t \rightarrow \infty}(4.27) & =\lim _{t \rightarrow \infty} \int_{\mathcal{B}} \mathbb{E}\left[F_{1}\left(\pi_{h}\left(\left(\varepsilon_{s}\right)_{s \geq 0}\right)\right) \mid \pi_{h}(X)=\nu\right] \mathbb{P}\left(\pi_{h}(X) \in d \nu \mid \mathcal{O}_{t}\right) \\
& \stackrel{3.14}{=} \int_{\mathcal{B}} \mathbb{E}\left[F_{1}\left(\pi_{h}\left(\left(\varepsilon_{s}\right)_{s \geq 0}\right)\right) \mid \pi_{h}(X)=\nu\right] \mathbb{Q}\left(\pi_{h}(X) \in d \nu\right) \\
& =: \mathbb{E}_{\mathbb{Q}^{\prime \prime}}\left[F_{1}\left(\pi_{h}\left(\left(\varepsilon_{s}\right)_{s \geq 0}\right)\right) \mathbb{1}_{\left\{\pi_{h}(X) \in \mathcal{B}\right\}}\right]
\end{aligned}
$$

where we can swap the order of limits and integration since $F_{1}$ is bounded. Taking $F_{1} \equiv 1$, it follows immediately that $\mathbb{Q}^{\prime \prime}(\cdot)$ and $\mathbb{Q}(\cdot)$ agree on $\mathcal{D}[0, \infty)$. The weak convergence as $t \rightarrow \infty$ of $\left(\left(M_{t}\right)_{t \geq 0},\left(X_{s}\right)_{s \geq 0}\right)$ under $\mathbb{P}\left(\cdot \mid \mathcal{O}_{t}\right)$ to $\left(\left(M_{t}\right)_{t \geq 0},\left(X_{s}\right)_{s \geq 0}\right)$ under $\mathbb{Q}^{\prime \prime}(\cdot)$ then follows immediately from the fact (see e.g. Bertoin, 1996, Ex. IV.6.3 or Kolb and Savov, 2016, p4113) that for all $x>0,\left(M_{t}\right)_{t>0}$ is uniquely determined by $\left(\varepsilon_{s}\right)_{s>0}$ and $\left(X_{s}\right)_{s>0}$, and both of $\left(\varepsilon_{s}\right)_{s>0}$ and $\left(X_{s}\right)_{s>0}$ have weak limits as determined in (4.28). That is, we construct $M$ pathwise via its excursions using $\left(X_{u}\right)_{u \geq 0}$ to determine the timing and length of each excursion, where we sample the excursions of $M$ using the excursion measure conditional on each excursion length. The fact that $M$ is recurrent under $\mathbb{Q}^{\prime \prime}(\cdot)$ follows immediately from this construction, since $X$ does not explode to infinity by Proposition 3.15.

4.2.2. Proof of Theorem 3.18. Lemmas 4.7, 4.8, 4.9, 4.10, proven in Sections 6, 7, are needed for the proof of Theorem 3.18.

Lemma 4.7. For each subordinator and $g=f^{-1}$ in case (ia), there exist $K, h_{0}>0$ such that for all $h>h_{0}$, with $\bar{\Pi}(y)=y^{-\alpha} L(y)$, uniformly in $y>K$,

$$
\mathbb{P}\left(X_{h} \in g(h) d y ; \mathcal{O}_{h}\right) \asymp y^{-1-\alpha} \frac{L(g(h) y)}{L(g(h))} \mathbb{P}\left(\mathcal{O}_{h}\right) d y .
$$

Lemma 4.8. For $\delta>0$ small enough that $0<f(0)<f(\delta)<1$, uniformly for all $h>0$ and $y>g(h+f(\delta))$,

$$
q_{h}(y) \asymp \Phi_{y}^{h}(f(A y)) \exp \left(-\int_{1}^{f(A y)} \bar{\Pi}(g(s)) d s\right) .
$$

Lemma 4.9. For a subordinator and $g=f^{-1}$ in case (ia), let $S_{\Delta_{1}^{g(h)}}$ denote the size of its first jump of size greater than $g(h)$. Then there is $h_{0}>0$ such that uniformly in $h>h_{0}$ and $v>1$,

$$
\mathbb{P}\left(S_{\Delta_{1}^{g(h)}} \in g(h) d v\right)=\frac{\Pi(g(h) d v)}{\bar{\Pi}(g(h))} \asymp \frac{L(g(h) v)}{L(g(h))} v^{-1-\alpha} d v .
$$

In particular there is $x_{0} \in(0, \infty)$ so that for all $x>x_{0}$, with $\Pi(d x)=u(x) d x$,

$$
u(x) \asymp x^{-1} \bar{\Pi}(x)=L(x) x^{-1-\alpha} .
$$

Lemma 4.10. Recall the notation (3.12),(3.20). If $h>0, y>g(h)$, and $t \geq$ $f(A y)$, for $A>3 \vee(B-1)$, then $\Phi_{y}^{h}(t) \geq f(y)-h$. 
Proof of Theorem 3.18: By Proposition 3.15, $\mathbb{Q}\left(X_{h} \in(g(h), \infty)\right)=1, h \geq 0$. By Definition 3.17,

$$
w \in R_{g} \Longleftrightarrow \lim _{h \rightarrow \infty} \mathbb{Q}\left(X_{h} \in(g(h), w(h) g(h))\right)=0,
$$

since $\mathbb{Q}^{\prime \prime}(\cdot)$ and $\mathbb{Q}(\cdot)$ agree on the space $\mathcal{D}[0, \infty)$ containing $X$. Now, by Theorem 3.14,

$$
\lim _{h \rightarrow \infty} \mathbb{Q}\left(X_{h} \in(g(h), w(h) g(h))\right)=\lim _{h \rightarrow \infty} \int_{g(h)}^{w(h) g(h)} q_{h}(y) \mathbb{P}\left(X_{h} \in d y ; \mathcal{O}_{h}\right) .
$$

We begin by showing that if $\lim _{h \rightarrow \infty} \int_{h}^{f(w(h) g(h))} \bar{\Pi}(g(s)) d s=0$, then $w \in R_{g}$.

Proof of Sufficient Condition. Let $\lim _{h \rightarrow \infty} \int_{h}^{f(w(h) g(h))} \bar{\Pi}(g(s)) d s=0$. To show $w \in R_{g}$, we will show that the limit of the integral in (4.32) is zero on each of

$[g(h), g(h+1)] \cup[g(h+1), K g(h)] \cup[g(h+1) \vee K g(h), w(h) g(h)]=: R_{1} \cup R_{2} \cup R_{3}$

separately, where $K$ is the constant as in Lemma 4.7. Note that if $g(h+1)>K g(h)$, we need only consider $R_{1} \cup R_{3}$. Since $g$ is non-decreasing, we only need to consider the value $K$ if $K>1$.

Proof for $R_{1}$. By Theorem 3.14, $q_{h}(y)$ is non-decreasing in $y$, so

$$
\begin{aligned}
& \lim _{h \rightarrow \infty} \int_{g(h)}^{g(h+1)} q_{h}(y) \mathbb{P}\left(X_{h} \in d y ; \mathcal{O}_{h}\right) \\
& \leq \lim _{h \rightarrow \infty} q_{h}(g(h+1)) \int_{g(h)}^{g(h+1)} \mathbb{P}\left(X_{h} \in d y ; \mathcal{O}_{h}\right) \leq \lim _{h \rightarrow \infty} q_{h}(g(h+1)) \mathbb{P}\left(\mathcal{O}_{h}\right) .
\end{aligned}
$$

Applying Lemma 4.8, then applying Corollary 4.4,

$$
\begin{aligned}
(4.33) & \lesssim \lim _{h \rightarrow \infty} \Phi_{g(h+1)}^{h}(f(A g(h+1))) e^{-\int_{1}^{f(A g(h+1))} \bar{\Pi}(g(s)) d s} \mathbb{P}\left(\mathcal{O}_{h}\right) \\
& =\lim _{h \rightarrow \infty} \Phi_{g(h+1)}^{h}(f(A g(h+1))) e^{-\int_{1}^{f(A g(h+1))} \bar{\Pi}(g(s)) d s} \Phi(h) \bar{\Pi}(g(h)) .
\end{aligned}
$$

Now, $\Phi_{g(h+1)}^{h}(f(A g(h+1))) \leq f(A g(h+1)) \lesssim h$, and $f(A g(h+1)) \geq h$, as $f=g^{-1}$ is $\mathcal{O}$-regularly varying, increasing, and $A>1$. By (4.3) and (3.1),

$$
\begin{aligned}
(4.33) & \lesssim \lim _{h \rightarrow \infty} h e^{-\int_{1}^{f(A g(h+1))} \bar{\Pi}(g(s)) d s} \Phi(h) \bar{\Pi}(g(h)) \\
& \leq \lim _{h \rightarrow \infty} h e^{-\int_{1}^{h} \bar{\Pi}(g(s)) d s} \Phi(h) \bar{\Pi}(g(h)) \stackrel{(4.3)}{\lesssim} \lim _{h \rightarrow \infty} h \bar{\Pi}(g(h)) \stackrel{(3.1)}{=} 0 .
\end{aligned}
$$

Proof for $R_{2}$. Recall that we only need to consider $R_{2}$ when $g(h+1)<K g(h)$, in which case $K$ must satisfy $K>1$. By Theorem 3.14, $q_{h}(y)$ is non-decreasing in $y$, so

$$
\begin{aligned}
& \lim _{h \rightarrow \infty} \int_{g(h+1)}^{K g(h)} q_{h}(y) \mathbb{P}\left(X_{h} \in d y ; \mathcal{O}_{h}\right) \\
& \leq \lim _{h \rightarrow \infty} q_{h}(K g(h)) \int_{g(h+1)}^{K g(h)} \mathbb{P}\left(X_{h} \in d y ; \mathcal{O}_{h}\right) \leq \lim _{h \rightarrow \infty} q_{h}(K g(h)) \mathbb{P}\left(\mathcal{O}_{h}\right) .
\end{aligned}
$$


Applying Corollary 4.4, then Lemma $4.8(g(h+f(\delta))<g(h+1)<K g(h)$ for $\delta$ in Lemma 4.8),

$$
\begin{aligned}
(4.34) & \stackrel{4.4}{\leq} \lim _{h \rightarrow \infty} q_{h}(K g(h)) \Phi(h) \bar{\Pi}(g(h)) . \\
& \stackrel{4.8}{\lesssim} \lim _{h \rightarrow \infty} \Phi_{K g(h)}^{h}(f(A K g(h))) e^{-\int_{1}^{f(A K g(h))} \bar{\Pi}(g(s)) d s} \Phi(h) \bar{\Pi}(g(h)) .
\end{aligned}
$$

Observe that $\Phi_{K g(h)}^{h}(t) \leq t$ for all $t>0$, by (3.12), and $f(A K g(h)) \geq f(g(h))=h$ since $f$ increasing and $A, K>1$. Moreover, as $f$ is $\mathcal{O}$-regularly varying at $\infty$, $f(A K g(h)) \lesssim f(g(h))=h$, as $h \rightarrow \infty$, so

$$
\begin{aligned}
(4.34) & \leq \lim _{h \rightarrow \infty} f(A K g(h)) \exp \left(-\int_{1}^{f(A K g(h))} \bar{\Pi}(g(s)) d s\right) \Phi(h) \bar{\Pi}(g(h)) . \\
& \leq \lim _{h \rightarrow \infty} f(A K g(h)) \exp \left(-\int_{1}^{h} \bar{\Pi}(g(s)) d s\right) \Phi(h) \bar{\Pi}(g(h)) \\
& \lesssim \lim _{h \rightarrow \infty} h \exp \left(-\int_{1}^{h} \bar{\Pi}(g(s)) d s\right) \Phi(h) \bar{\Pi}(g(h)) .
\end{aligned}
$$

By (4.3), $\Phi(h) \asymp \exp \left(\int_{1}^{h} \bar{\Pi}(g(s)) d s\right)$ as $h \rightarrow \infty$, so as $\lim _{h \rightarrow \infty} h \bar{\Pi}(g(h))=0$ by (3.1), we conclude that (4.34) $=0$, so the integral over $R_{2}$ is zero, and thus

$$
\lim _{h \rightarrow \infty} \int_{R_{1} \cup R_{2}} q_{h}(y) \mathbb{P}\left(X_{h} \in d y ; \mathcal{O}_{h}\right)=0 .
$$

Proof for $R_{3}$. Now we wish to show convergence to zero of

$$
\int_{g(h+1) \vee K g(h)}^{w(h) g(h)} q_{h}(y) \mathbb{P}\left(X_{h} \in d y ; \mathcal{O}_{h}\right)=\int_{\frac{g(h+1)}{g(h)} \vee K}^{w(h)} q_{h}(g(h) v) \mathbb{P}\left(X_{h} \in g(h) d v ; \mathcal{O}_{h}\right) .
$$

Applying Lemma 4.7, then changing variables back to $y=g(h) v$, recalling that $\bar{\Pi}(g(h))=g(h)^{-\alpha} L(g(h))$, then applying Corollary 4.4, as $h \rightarrow \infty$,

$$
\begin{aligned}
(4.36) & \stackrel{4.7}{\asymp} \int_{\frac{g(h+1)}{g(h)} \vee K}^{w(h)} q_{h}(g(h) v) v^{-1-\alpha} \frac{L(g(h) v)}{L(g(h))} \mathbb{P}\left(\mathcal{O}_{h}\right) d v \\
& =\frac{\mathbb{P}\left(\mathcal{O}_{h}\right) g(h)^{\alpha}}{L(g(h))} \int_{\frac{g(h+1)}{g(h)} \vee K}^{w(h)} q_{h}(g(h) v) g(h)^{-\alpha} v^{-1-\alpha} L(g(h) v) d v \\
& =\frac{\mathbb{P}\left(\mathcal{O}_{h}\right)}{\bar{\Pi}(g(h))} \int_{g(h+1) \vee K g(h)}^{w(h) g(h)} q_{h}(y) y^{-1-\alpha} L(y) d y \\
& \stackrel{4.4}{\sim} \Phi(h) \int_{g(h+1) \vee K g(h)}^{w(h) g(h)} q_{h}(y) y^{-1-\alpha} L(y) d y .
\end{aligned}
$$

Changing variables $(u=A y)$, applying Lemma 4.8 and then the uniform convergence theorem (Bingham et al., 1989, Theorem 1.2.1), as $L$ is slowly varying at $\infty$ and $\Phi_{y}^{h}(f(u)) \leq f(u)$, it follows that as $h \rightarrow \infty$,

$$
(4.36) \stackrel{4.8}{\longleftarrow} \Phi(h) \int_{g(h+1) \vee K g(h)}^{w(h) g(h)} \Phi_{y}^{h}(f(A y)) \exp \left(-\int_{1}^{f(A y)} \bar{\Pi}(g(s)) d s\right) y^{-1-\alpha} L(y) d y
$$




$$
\begin{aligned}
& \leq \Phi(h) \int_{K g(h)}^{w(h) g(h)} \Phi_{y}^{h}(f(A y)) \exp \left(-\int_{1}^{f(A y)} \bar{\Pi}(g(s)) d s\right) y^{-1-\alpha} L(y) d y \\
& \lesssim \Phi(h) \int_{A K g(h)}^{A w(h) g(h)} \Phi_{y}^{h}(f(u)) \exp \left(-\int_{1}^{f(u)} \bar{\Pi}(g(s)) d s\right) u^{-1-\alpha} L(u) d u \\
& \leq \Phi(h) \int_{A K g(h)}^{A w(h) g(h)} f(u) \exp \left(-\int_{1}^{f(u)} \bar{\Pi}(g(s)) d s\right) u^{-1-\alpha} L(u) d u .
\end{aligned}
$$

Since $A, K>1$, we can split up the integral as follows, and we will deal with each term separately:

$$
\begin{aligned}
(4.36) & \lesssim \Phi(h) \int_{g(h)}^{w(h) g(h)} f(u) \exp \left(-\int_{1}^{f(u)} \bar{\Pi}(g(s)) d s\right) u^{-1-\alpha} L(u) d u \\
& +\Phi(h) \int_{w(h) g(h)}^{A w(h) g(h)} f(u) \exp \left(-\int_{1}^{f(u)} \bar{\Pi}(g(s)) d s\right) u^{-1-\alpha} L(u) d u \\
& =: J_{1}(h)+J_{2}(h) .
\end{aligned}
$$

Proof for $J_{2}(h)$. As $f(u) \geq f(w(h) g(h)) \geq f(g(h))=h$ for $u \geq w(h) g(h)$, by (4.3),

$$
\begin{aligned}
J_{2}(h) & =\Phi(h) \int_{w(h) g(h)}^{A w(h) g(h)} f(u) e^{-\int_{1}^{f(u)} \bar{\Pi}(g(s)) d s} u^{-1-\alpha} L(u) d u \\
& =\Phi(h) e^{-\int_{1}^{h} \bar{\Pi}(g(s)) d s} \int_{w(h) g(h)}^{A w(h) g(h)} f(u) e^{-\int_{h}^{f(u)} \bar{\Pi}(g(s)) d s} u^{-1-\alpha} L(u) d u \\
& \stackrel{(4.3)}{\lesssim} \int_{w(h) g(h)}^{A w(h) g(h)} f(u) e^{-\int_{h}^{f(u)} \bar{\Pi}(g(s)) d s} u^{-1-\alpha} L(u) d u \\
& \leq \int_{w(h) g(h)}^{A w(h) g(h)} f(u) u^{-1-\alpha} L(u) d u .
\end{aligned}
$$

Since $f, f^{\prime}$ are $\mathcal{O}$-regularly varying at $\infty$, one can verify that, uniformly for all sufficiently large $u, f(u) / u \asymp f^{\prime}(u)$, see Bingham et al. (1989, Prop 2.10.3). Recall that in case (i), $u f^{\prime}(u) \bar{\Pi}(u)$ decreases to 0 as $u \rightarrow \infty$, so as $h \rightarrow \infty$,

$$
\begin{aligned}
J_{2}(h) & \lesssim \int_{w(h) g(h)}^{A w(h) g(h)} f^{\prime}(u) u^{-\alpha} L(u) d u=\int_{w(h) g(h)}^{A w(h) g(h)} u f^{\prime}(u) \bar{\Pi}(u) u^{-1} d u . \\
& \leq w(h) g(h) f^{\prime}(w(h) g(h)) \bar{\Pi}(w(h) g(h)) \int_{w(h) g(h)}^{A w(h) g(h)} u^{-1} d u \\
& =o(1) \times \int_{w(h) g(h)}^{A w(h) g(h)} u^{-1} d u=o(1) \times \log (A)=o(1),
\end{aligned}
$$

so $\lim _{h \rightarrow \infty} J_{2}(h)=0$, and $J_{2}(h)$ never contributes. Now we consider $J_{1}(h)$.

Proof for $J_{1}(h)$. First, changing variables from $s$ to $v:=g(s)$, so that $s=f(v)$,

$$
J_{1}(h)=\Phi(h) \int_{g(h)}^{w(h) g(h)} f(u) \exp \left(-\int_{1}^{f(u)} \bar{\Pi}(g(s)) d s\right) u^{-1-\alpha} L(u) d u
$$




$$
=\Phi(h) \int_{g(h)}^{w(h) g(h)} f(u) \exp \left(-\int_{g(1)}^{u} \bar{\Pi}(v) f^{\prime}(v) d v\right) u^{-1-\alpha} L(u) d u .
$$

Recall $u^{-\alpha} L(u)=\bar{\Pi}(u)$, and $f(u) \asymp u f^{\prime}(u)$ uniformly as $u \rightarrow \infty$, so as $h \rightarrow \infty$,

$$
\begin{aligned}
J_{1}(h) & \asymp \Phi(h) \int_{g(h)}^{w(h) g(h)} f^{\prime}(u) \exp \left(-\int_{g(1)}^{u} \bar{\Pi}(v) f^{\prime}(v) d v\right) u^{-\alpha} L(u) d u \\
& =\Phi(h) \int_{g(h)}^{w(h) g(h)} f^{\prime}(u) \bar{\Pi}(u) \exp \left(-\int_{g(1)}^{u} \bar{\Pi}(v) f^{\prime}(v) d v\right) d u
\end{aligned}
$$

Changing variables from $u$ to $z:=e^{-\int_{g(1)}^{u} \bar{\Pi}(v) f^{\prime}(v) d v}$ and applying (4.3), it follows that as $h \rightarrow \infty$,

$$
\begin{aligned}
J_{1}(h) & \asymp \Phi(h)\left[e^{-\int_{g(1)}^{g(h)} \bar{\Pi}(v) f^{\prime}(v) d v}-e^{-\int_{g(1)}^{w(h) g(h)} \bar{\Pi}(v) f^{\prime}(v) d v}\right] \\
& =\Phi(h)\left[e^{-\int_{1}^{h} \bar{\Pi}(g(s)) d s}-e^{-\int_{1}^{f(w(h) g(h))} \bar{\Pi}(g(s)) d s}\right] \\
& \stackrel{(4.3)}{\asymp} 1-e^{-\int_{h}^{f(w(h) g(h))} \bar{\Pi}(g(s)) d s} .
\end{aligned}
$$

Thus by (4.35), (4.38) and (4.39), whenever $\lim _{h \rightarrow \infty} \int_{h}^{f(w(h) g(h))} \bar{\Pi}(g(s)) d s=0, w$ is in the entropic repulsion envelope $R_{g}$, as required for the sufficient condition.

Now we will prove that if $w \in R_{g}$, then $\lim _{h \rightarrow \infty} \int_{h}^{f(w(h) g(h))} \bar{\Pi}(g(s)) d s=0$. Proof of Necessary Condition. Let $w \in R_{g}$. Then by (3.23),

$$
\begin{aligned}
0 & =\lim _{h \rightarrow \infty} \mathbb{Q}\left(X_{h} \in(g(h), w(h) g(h))\right) \\
& =\lim _{h \rightarrow \infty} \int_{g(h)}^{w(h) g(h)} q_{h}(y) \mathbb{P}\left(X_{h} \in d y ; \mathcal{O}_{h}\right)=\lim _{h \rightarrow \infty} \int_{K g(h)}^{w(h) g(h)} q_{h}(y) \mathbb{P}\left(X_{h} \in d y ; \mathcal{O}_{h}\right),
\end{aligned}
$$

since the limit of the integral over $R_{1} \cup R_{2}=(g(h), K g(h))$ is always zero by (4.35), regardless of $\lim _{h \rightarrow \infty} \int_{h}^{f(w(h) g(h))} \bar{\Pi}(g(s)) d s=0$. Changing variables to $v=y / g(h)$ and applying Lemma 4.7,

$$
\begin{aligned}
0 & =\lim _{h \rightarrow \infty} \int_{K}^{w(h)} q_{h}(g(h) v) \mathbb{P}\left(X_{h} \in g(h) d v ; \mathcal{O}_{h}\right) \\
& \stackrel{4.7}{=} \lim _{h \rightarrow \infty} \mathbb{P}\left(\mathcal{O}_{h}\right) \int_{K}^{w(h)} q_{h}(g(h) v) v^{-1-\alpha} \frac{L(g(h) v)}{L(g(h))} d v .
\end{aligned}
$$

Changing variables to $y=g(h) v$ and recallling that $\bar{\Pi}(g(h))=g(h)^{-\alpha} L(g(h))$, then applying Corollary 4.4, and Lemmas 4.8 and 4.10,

$$
\begin{aligned}
0 & =\lim _{h \rightarrow \infty} \frac{\mathbb{P}\left(\mathcal{O}_{h}\right)}{\bar{\Pi}(g(h))} \int_{K g(h)}^{w(h) g(h)} q_{h}(y) y^{-1-\alpha} L(y) d y \\
& \stackrel{4.4}{=} \lim _{h \rightarrow \infty} \Phi(h) \int_{K g(h)}^{w(h) g(h)} q_{h}(y) y^{-1-\alpha} L(y) d y \\
& \stackrel{4.8}{=} \lim _{h \rightarrow \infty} \Phi(h) \int_{K g(h)}^{w(h) g(h)} \Phi_{y}^{h}(f(A y)) e^{-\int_{1}^{f(A y)} \bar{\Pi}(g(s)) d s} y^{-1-\alpha} L(y) d y
\end{aligned}
$$




$$
\begin{aligned}
& \stackrel{4.10}{\geq} \lim _{h \rightarrow \infty}\left[\Phi(h) \int_{K g(h)}^{w(h) g(h)} f(y) e^{-\int_{1}^{f(A y)} \bar{\Pi}(g(s)) d s} y^{-1-\alpha} L(y) d y\right. \\
& \left.-h \Phi(h) \int_{K g(h)}^{w(h) g(h)} e^{-\int_{1}^{f(A y)} \bar{\Pi}(g(s)) d s} y^{-1-\alpha} L(y) d y\right] \\
& =: \lim _{h \rightarrow \infty}\left[I_{1}-I_{2}\right] \text {. }
\end{aligned}
$$

First we consider $I_{2}$. Note that $A K>1$, so $f(A y) \geq f(A K g(h)) \geq h$ for all $y \geq K g(h)$. Then since $\Phi(h) \asymp e^{-\int_{1}^{h} \bar{\Pi}(g(s)) d s}$ by (4.3),

$$
\begin{aligned}
\lim _{h \rightarrow \infty}\left|I_{2}\right| & \leq \lim _{h \rightarrow \infty} h \Phi(h) \int_{K g(h)}^{w(h) g(h)} e^{-\int_{1}^{h} \bar{\Pi}(g(s)) d s} y^{-1-\alpha} L(y) d y \\
& \stackrel{(4.3)}{\lesssim} \lim _{h \rightarrow \infty} h \int_{K g(h)}^{w(h) g(h)} y^{-1-\alpha} L(y) d y .
\end{aligned}
$$

By Lemma 4.9, $y^{-1-\alpha} L(y) d y \asymp \Pi(d y)$, so as $\bar{\Pi}$ is regularly varying at $\infty$, by (3.1),

$$
\lim _{h \rightarrow \infty}\left|I_{2}\right| \stackrel{4.9}{\lesssim} \lim _{h \rightarrow \infty} h \int_{K g(h)}^{w(h) g(h)} \Pi(d y) \leq \lim _{h \rightarrow \infty} h \bar{\Pi}(K g(h)) \lesssim \lim _{h \rightarrow \infty} h \bar{\Pi}(g(h)) \stackrel{(3.1)}{=} 0,
$$

so $I_{2}=0$, and thus $\lim _{h \rightarrow \infty} I_{1} \leq 0$. As $I_{1}$ is non-negative, $\lim _{h \rightarrow \infty} I_{1}=0$. Now, changing variables to $v:=A y$, as $f$ is $\mathcal{O}$-regularly varying at $\infty$ and $L$ is slowly varying at $\infty$, by the uniform convergence theorem Bingham et al. (1989, Theorem 1.2.1),

$$
\begin{aligned}
0=\lim _{h \rightarrow \infty} I_{1} & =\lim _{h \rightarrow \infty} \Phi(h) \int_{A K g(h)}^{A w(h) g(h)} f\left(\frac{v}{A}\right) e^{-\int_{1}^{f(v)} \bar{\Pi}(g(s)) d s} v^{-1-\alpha} A^{\alpha} L\left(\frac{v}{A}\right) d v \\
& \asymp \lim _{h \rightarrow \infty} \Phi(h) \int_{A K g(h)}^{A w(h) g(h)} f(v) e^{-\int_{1}^{f(v)} \bar{\Pi}(g(s)) d s} v^{-1-\alpha} L(v) d v .
\end{aligned}
$$

Recall $v^{-\alpha} L(v)=\bar{\Pi}(v)$, and $f(v) \asymp v f^{\prime}(v)$ uniformly for all large enough $v$, because $f, f^{\prime}$ are $\mathcal{O}$-regularly varying at $\infty$, see Bingham et al. (1989, Prop 2.10.3). Then

$$
\begin{aligned}
0 & =\lim _{h \rightarrow \infty} \Phi(h) \int_{A K g(h)}^{A w(h) g(h)} f(v) e^{-\int_{1}^{f(v)} \bar{\Pi}(g(s)) d s} v^{-1} \bar{\Pi}(v) d v \\
& \asymp \lim _{h \rightarrow \infty} \Phi(h) \int_{A K g(h)}^{A w(h) g(h)} f^{\prime}(v) \bar{\Pi}(v) e^{-\int_{1}^{f(v)} \bar{\Pi}(g(s)) d s} d v .
\end{aligned}
$$

Now, one can verify that $P(v):=\int_{g(1)}^{v} \bar{\Pi}(u) f^{\prime}(u) d u=\int_{1}^{f(v)} \bar{\Pi}(g(s)) d s$ by changing variables from $u$ to $s=f(u)$. Then as $A>3$ and $P^{\prime}(v)=\bar{\Pi}(v) f^{\prime}(v) \geq 0$,

$$
\begin{aligned}
0 & =\lim _{h \rightarrow \infty} \Phi(h) \int_{A K g(h)}^{A w(h) g(h)} P^{\prime}(v) e^{-P(v)} d v \\
& \geq \lim _{h \rightarrow \infty}\left[\Phi(h) \int_{g(h)}^{w(h) g(h)} P^{\prime}(v) e^{-P(v)} d v-\Phi(h) \int_{g(h)}^{A K g(h)} P^{\prime}(v) e^{-P(v)} d v\right] \\
& =: \lim _{h \rightarrow \infty}\left[K_{1}-K_{2}\right] .
\end{aligned}
$$


Now, recall that by (4.39), as $h \rightarrow \infty$, we have

$$
K_{1} \asymp J_{1} \asymp\left(1-e^{-\int_{h}^{f(w(h) g(h))} \bar{\Pi}(g(s)) d s}\right) .
$$

So if we prove $\lim _{h \rightarrow \infty} K_{1}=0$, then $\lim _{h \rightarrow \infty} \int_{h}^{f(w(h) g(h))} \bar{\Pi}(g(s)) d s=0$, and the proof is complete. As $K_{1}$ is always non-negative, it suffices to prove that $\lim _{h \rightarrow \infty} K_{1} \leq 0$. To prove this, we will show that $\lim _{h \rightarrow \infty}\left|K_{2}\right|=0$. Since $g=f^{-1}$, note $f(v)>h$ for $v>g(h)$, then as $\Phi(h) \asymp e^{\int_{1}^{h} \bar{\Pi}(g(s)) d s}$ by (4.3),

$$
\begin{aligned}
\lim _{h \rightarrow \infty}\left|K_{2}\right| & =\lim _{h \rightarrow \infty} \Phi(h) \int_{g(h)}^{A K g(h)} \bar{\Pi}(v) f^{\prime}(v) e^{-\int_{1}^{f(v)} \bar{\Pi}(g(s)) d s} d v \\
& \stackrel{(4.3)}{\longleftarrow} \lim _{h \rightarrow \infty} \int_{g(h)}^{A K g(h)} \bar{\Pi}(v) f^{\prime}(v) e^{-\int_{h}^{f(v)} \bar{\Pi}(g(s)) d s} d v \\
& \leq \lim _{h \rightarrow \infty} \int_{g(h)}^{A K g(h)} v f^{\prime}(v) \bar{\Pi}(v) v^{-1} d v .
\end{aligned}
$$

Recall that by assumption, $v f^{\prime}(v) \bar{\Pi}(v)$ decreases to 0 as $v \rightarrow \infty$, and hence

$$
\begin{aligned}
\lim _{h \rightarrow \infty}\left|K_{2}\right| & \lesssim \lim _{h \rightarrow \infty} g(h) f^{\prime}(g(h)) \bar{\Pi}(g(h)) \int_{g(h)}^{A K g(h)} v^{-1} d v \\
& =\lim _{h \rightarrow \infty} g(h) f^{\prime}(g(h)) \bar{\Pi}(g(h)) \times \log (A K)=0 .
\end{aligned}
$$

\subsubsection{Proof of Corollary 3.19.}

Proof of Corollary 3.19: We need to verify that a stable subordinator of index $\alpha \in$ $(0,1)$ satisfies (3.2), so Theorem 3.18 applies. For $t>0$ and $x>g(t)+x_{0}$, by the scaling property of stable subordinators (see Bertoin, 1996, p227),

$$
f_{t}(x)=t^{-\frac{1}{\alpha}} f_{1}\left(\frac{x}{t^{\frac{1}{\alpha}}}\right) .
$$

Now consider the result (Nolan, 2018, Theorem 1.12) that for a stable subordinator of index $\alpha \in(0,1), f_{1}(v) \sim c_{\alpha} v^{-1-\alpha}$ as $v \rightarrow \infty$, for $c_{\alpha}>0$ constant. In particular, for all large enough $v, f_{1}(v)$ is arbitrarily close to $c_{\alpha} v^{-1-\alpha}$. Taking e.g. $a^{\prime}=2 c_{\alpha}$, it follows that there exist $a^{\prime}, C \in(0, \infty)$ such that for all $v>C, f_{1}(v) \leq a^{\prime} v^{-1-\alpha}$.

As $\Pi(d v)=u(v) d v=c v^{-1-\alpha} d v$ for a constant $c>0$, if we can show that $x / t^{1 / \alpha} \geq C$ for all $t>0, x>g(t)+x_{0}$, with a suitable choice of $x_{0}>0$, then by (4.41),

$$
f_{t}(x)=t^{-\frac{1}{\alpha}} f_{1}\left(\frac{x}{t^{\frac{1}{\alpha}}}\right) \leq a^{\prime} c t x^{-1-\alpha}=a t u(x),
$$

for $a:=a^{\prime} c$, so condition (3.2) is satisfied, and the proof will be complete. Indeed, by (3.1), we have $\lim _{t \rightarrow \infty} t \bar{\Pi}(g(t))=\lim _{t \rightarrow \infty} t g(t)^{-\alpha}=0$, so there exists $D \in(0, \infty)$ such that for all $t>D, \operatorname{tg}(t)^{-\alpha} \leq C^{-\alpha}$, so $t^{1 / \alpha} \leq C^{-1} g(t)$, and hence for all $t>D$,

$$
\frac{x}{t^{\frac{1}{\alpha}}} \geq \frac{g(t)+x_{0}}{t^{\frac{1}{\alpha}}} \geq \frac{g(t)}{t^{\frac{1}{\alpha}}} \geq C .
$$

On the other hand, if $t \leq D$, then $x / t^{1 / \alpha} \geq\left(g(t)+x_{0}\right) / D^{1 / \alpha} \geq x_{0} / D^{1 / \alpha}$, and (choosing $x_{0}$ large enough that $x_{0} / D^{1 / \alpha}>C$ if necessary), we conclude that 
$x / t^{1 / \alpha}>C$ for all $t>0, x>g(t)+x_{0}$, so Nolan (2018, Theorem 1.12) applies to (4.41). It follows that condition (3.2) is satisfied, and so Theorem 3.18 applies, as required.

\section{Proof of Lemma 4.2}

In order to prove Lemma 4.2, we require Lemmas 5.1 and 5.2. The proof of Lemma 5.1 is provided in Section 7, whereas the proof of Lemma 5.2 is provided immediately below.

Lemma 5.1. Let $\left(X_{t}\right)_{t \geq 0}$ be a subordinator satisfying the assumptions in case (i) or (ii). Then there exists a constant $C>0$, which depends only on the law of $X$, such that for all $t>0, A(t) \in(1, \infty), B(t)>0$, and $H(t) \in(0,1)$,

$$
\mathbb{P}\left(X_{t}^{(0, A(t))}>B(t)\right) \leq \exp \left(C t \log \left(\frac{1}{H(t)}\right) H(t)^{-\frac{A(t)}{B(t)}} \bar{\Pi}(A(t)) \frac{A(t)}{B(t)}\right) H(t) .
$$

Lemma 5.2. Recalling (3.10) and (3.20), if $t>t_{0}(y), h \geq 0$, and $y \geq 0$, then $g_{y}^{h}(t) \geq(1-1 / A) g(t)$.

Proof of Lemma 5.2: By (3.20), $t>t_{0}(y) \geq f(A y)$. The result holds trivially when $y=0$. Otherwise, as $g=f^{-1}$ is increasing,

$$
\begin{aligned}
g_{y}^{h}(t) & =\left(\frac{g(t+h)}{g(t)}-\frac{y}{g(t)}\right) g(t) \geq\left(1-\frac{y}{g(t)}\right) g(t) \\
& \geq\left(1-\frac{y}{g(f(A y))}\right) g(t)=\left(1-A^{-1}\right) g(t) .
\end{aligned}
$$

Now, in addition to showing that for each $A>3$, the inequalities (4.1) and (4.2) hold uniformly in $h>0, y>g(h)$, and $t>t_{0}(y)$, we shall show that the inequalities (4.1) and (4.2) hold when $y=h=0$ for $t>t_{0}(0)>0$, with $\rho(t)$ in place of $\rho_{y}^{h}(t)$. For brevity, let us introduce the following notation:

$$
\mathcal{S}:=\left\{(h, y, t) \in \mathbb{R}^{3}: h>0, y>g(h), t>t_{0}(y)\right\} \cup\left\{(0,0, t) \in \mathbb{R}^{3}: t>t_{0}(0)\right\} .
$$

Lemma 4.2 shall be proven by splitting up $\rho_{y}^{h}(t)$ into smaller pieces, and then showing that the inequalities (4.1) and (4.2) hold for each piece separately.

Proof of Lemma 4.2: Lemma 4.2 is simpler to prove in case (ii) than case (i) thanks to the condition (3.3). We thus omit the proof in case (ii). Firstly, since $g=f^{-1}$ is continuous (and hence so is $g_{y}^{h}$ ), for all $h, y \geq 0$ and $x, t>0$,

$$
\mathbb{P}\left(\mathcal{O}_{t, X^{(0, x)}}^{g_{y}^{h}}\right)=\mathbb{P}\left(\mathcal{O}_{t-, X^{(0, x)}}^{g_{y}^{h}}\right),
$$

where $\mathcal{O}_{t-, X^{(0, x)}}^{g_{y}^{h}}:=\bigcap_{u<t} \mathcal{O}_{u, X^{(0, x)}}^{g_{y}^{h}}$. Moreover, by (3.6), for all $h, y \geq 0, x>0$, and $t \geq s>0$,

$$
\mathbb{P}\left(\mathcal{O}_{t, X^{(0, x)}}^{g_{y}^{h}}\right) \leq \mathbb{P}\left(\mathcal{O}_{s, X^{(0, x)}}^{g_{y}^{h}} ; X_{t}^{(0, x)} \geq g_{y}^{h}(t)\right) \leq \mathbb{P}\left(X_{t}^{(0, x)}>g_{y}^{h}(t)\right) .
$$


Now, we partition and disintegrate on the value of $\Delta_{1}^{g_{y}^{h}(t)}$, which is exponentially distributed with rate $\bar{\Pi}\left(g_{y}^{h}(t)\right)$. It follows by (3.11), (5.3) and (3.6), since $s<t$, that for all $h, y \geq 0$ and $t>0$,

$$
\begin{aligned}
\mathbb{P}\left(\mathcal{O}_{t}^{g_{y}^{h}} \mid \Delta_{1}^{g_{y}^{h}(t)}=s\right) & =\mathbb{P}\left(\mathcal{O}_{s}^{g_{y}^{h}} \mid \Delta_{1}^{g_{y}^{h}(t)}=s\right)=\mathbb{P}\left(\mathcal{O}_{s-}^{g_{y}^{h}} \mid \Delta_{1}^{g_{y}^{h}(t)}\right. \\
=s & =\mathbb{P}\left(\mathcal{O}_{s-, X_{y}^{\left(0, g_{y}^{h}(t)\right)}}^{g_{y}^{h}}\right)=\mathbb{P}\left(\mathcal{O}_{s, X^{\left(0, g_{y}^{h}(t)\right)}}^{g_{y}^{h}}\right),
\end{aligned}
$$

where the last equality holds since the small and large jumps are independent, so it follows that

$$
\begin{aligned}
\mathbb{P}\left(\mathcal{O}_{t}^{g_{y}^{h}}\right) & =\mathbb{P}\left(\mathcal{O}_{t}^{g_{y}^{h}} ; \Delta_{1}^{g_{y}^{h}(t)} \leq t\right)+\mathbb{P}\left(\mathcal{O}_{t}^{g_{y}^{h}} ; \Delta_{1}^{g_{y}^{h}(t)}>t\right) \\
& =\bar{\Pi}\left(g_{y}^{h}(t)\right) \int_{0}^{t} \mathbb{P}\left(\mathcal{O}_{t}^{g_{y}^{h}} \mid \Delta_{1}^{g_{y}^{h}(t)}=s\right) e^{-\bar{\Pi}\left(g_{y}^{h}(t)\right) s} d s+\mathbb{P}\left(\mathcal{O}_{t}^{g_{y}^{h}} ; \Delta_{1}^{g_{y}^{h}(t)}>t\right) \\
& =\bar{\Pi}\left(g_{y}^{h}(t)\right) \int_{0}^{t} \mathbb{P}\left(\mathcal{O}_{s, X^{\left(0, g_{y}^{h}(t)\right)}}^{g_{y}^{h}}\right) e^{-\bar{\Pi}\left(g_{y}^{h}(t)\right) s} d s+\mathbb{P}\left(\mathcal{O}_{t}^{g_{y}^{h}} ; \Delta_{1}^{g_{y}^{h}(t)}>t\right) .
\end{aligned}
$$

Now, observe that by the definition (3.6), $\mathbb{P}\left(\mathcal{O}_{s}^{g_{y}^{h}} \mid \Delta_{1}^{g_{y}^{h}(t)}>s\right)=\mathbb{P}\left(\mathcal{O}_{s, X^{\left(0, g_{y}^{h}(t)\right)}}^{g^{h}}\right)$, so for all $h, y \geq 0$,

$$
\begin{aligned}
\mathbb{P}\left(\mathcal{O}_{s}^{g_{y}^{h}}\right) & =\mathbb{P}\left(\mathcal{O}_{s}^{g_{y}^{h}} ; \Delta_{1}^{g_{y}^{h}(t)} \leq s\right)+\mathbb{P}\left(\mathcal{O}_{s}^{g_{y}^{h}} ; \Delta_{1}^{g_{y}^{h}(t)}>s\right) \\
& =\mathbb{P}\left(\mathcal{O}_{s}^{g_{y}^{h}} ; \Delta_{1}^{g_{y}^{h}(t)} \leq s\right)+\mathbb{P}\left(\mathcal{O}_{s}^{g_{y}^{h}} \mid \Delta_{1}^{g_{y}^{h}(t)}>s\right) \mathbb{P}\left(\Delta_{1}^{g_{y}^{h}(t)}>s\right) \\
& =\mathbb{P}\left(\mathcal{O}_{s}^{g_{y}^{h}} ; \Delta_{1}^{g_{y}^{h}(t)} \leq s\right)+\mathbb{P}\left(\begin{array}{c}
\mathcal{O}^{g_{y}^{h}} \\
s, X^{\left(0, g_{y}^{h}(t)\right)}
\end{array}\right) e^{-\bar{\Pi}\left(g_{y}^{h}(t)\right) s} .
\end{aligned}
$$

Disintegrating on $\Delta_{1}^{g_{y}^{h}(t)}$, recalling the notation introduced in (3.7) and (3.12), by (5.6), (5.7), and (5.3),

$$
\begin{aligned}
& \mathbb{P}\left(\mathcal{O}_{t}^{g_{y}^{h}}\right)=\bar{\Pi}\left(g_{y}^{h}(t)\right) \int_{0}^{t}\left[\mathbb{P}\left(\mathcal{O}_{s}^{g_{y}^{h}}\right)-\mathbb{P}\left(\mathcal{O}_{s}^{g_{y}^{h}} ; \Delta_{1}^{g_{y}^{h}(t)} \leq s\right)\right] d s+\mathbb{P}\left(\mathcal{O}_{t}^{g_{y}^{h}} ; \Delta_{1}^{g_{y}^{h}(t)}>t\right) \\
& \stackrel{(5.3)}{=} \bar{\Pi}\left(g_{y}^{h}(t)\right) \Phi(t)-\bar{\Pi}\left(g_{y}^{h}(t)\right)^{2} \int_{0}^{t} \int_{0}^{s} \mathbb{P}\left(\mathcal{O}_{v, X^{\left(0, g_{y}^{h}(t)\right)}}^{g^{h}}\right) e^{-\bar{\Pi}\left(g_{y}^{h}(t)\right) v} d v d s \\
& +\mathbb{P}\left(\mathcal{O}_{t}^{g_{y}^{h}} ; \Delta_{1}^{g_{y}^{h}(t)}>t\right) \\
& \leq \bar{\Pi}\left(g_{y}^{h}(t)\right) \Phi_{y}^{h}(t)+\mathbb{P}\left(\mathcal{O}_{t, X^{\left(0, g_{y}^{h}(t)\right)}}^{g^{h}}\right) \\
& -\bar{\Pi}\left(g_{y}^{h}(t)\right)^{2} \int_{0}^{t} \int_{0}^{s} \mathbb{P}\left(\mathcal{O}_{v}^{g_{y}^{h}}\right) e^{-\bar{\Pi}\left(g_{y}^{h}(t)\right) v} d v d s \\
& \leq \bar{\Pi}\left(g_{y}^{h}(t)\right) \Phi_{y}^{h}(t)+\mathbb{P}\left(\mathcal{O}_{t, X^{\left(0, g_{y}^{h}(t)\right)}}^{g_{y}^{h}}\right) .
\end{aligned}
$$

Recall the notation (3.7), (3.8), and (3.14). By (5.9), partitioning on the value of $\Delta_{1}^{\left(\frac{g_{y}^{h}(t)}{\log (t)}, g_{y}^{h}(t)\right)}$,

$\rho_{y}^{h}(t):=\frac{\mathbb{P}\left(\mathcal{O}_{t}^{g_{y}^{h}}\right)}{\Phi_{y}^{h}(t)}-\bar{\Pi}\left(g_{y}^{h}(t)\right) \stackrel{(5.9)}{\leq} \frac{1}{\Phi_{y}^{h}(t)} \mathbb{P}\left(\mathcal{O}_{t, X^{\left(0, g_{y}^{h}(t)\right)}}^{g^{h}}\right)$ 


$$
\begin{aligned}
& =\frac{1}{\Phi_{y}^{h}(t)}\left[\mathbb{P}\left(\mathcal{O}_{t, X^{\left(0, g_{y}^{h}(t)\right)}}^{g_{y}^{h}} ; \Delta_{1}^{\frac{g_{y}^{h}(t)}{\log (t)}}>t\right)+\mathbb{P}\left(\mathcal{O}_{t, X^{\left(0, g_{y}^{h}(t)\right)}}^{g_{y}^{h}} ; \Delta_{1}^{\left(\frac{g_{y}^{h}(t)}{\log (t)}, g_{y}^{h}(t)\right)} \leq t\right)\right] \\
& =: \frac{1}{\Phi_{y}^{h}(t)}[(a)+(b)] .
\end{aligned}
$$

So to prove (4.1), we need to prove, uniformly in $(h, y, t) \in \mathcal{S}$,

$$
(a)+(b) \lesssim \frac{\Phi_{y}^{h}(t)}{t \log (t)^{1+\varepsilon}}\left(1+\frac{1}{f(y)-h}\right) .
$$

For (4.2), we need suitable $u$ so that uniformly in $(h, y, t) \in \mathcal{S}$,

$$
(a)+(b) \leq \Phi_{y}^{h}(t) u(t) \bar{\Pi}(g(t))\left(1+\frac{1}{f(y)-h}\right) .
$$

Proof for (a). Recall the notation (3.6) and (3.11). By (5.4),

$$
(a)=\mathbb{P}\left(\mathcal{O}_{t, X^{\left(0, g_{y}^{h}(t)\right)}}^{g^{h}} ; \Delta_{1}^{\frac{g_{y}^{h}(t)}{\log (t)}}>t\right) \stackrel{(5.4)}{\leq} \mathbb{P}\left(X_{t}^{\left(0, \frac{g_{y}^{h}(t)}{\log (t)}\right)}>g_{y}^{h}(t)\right)
$$

To bound (5.13), we shall bound $\mathbb{P}\left(X_{t}^{\left(0, \frac{g_{y}^{h}(t)}{\log (t)}\right)}>K g_{y}^{h}(t)\right)$, with $K \in(0,1]$, giving a more general bound which will also be used later in the proof of Lemma 4.2. By Lemma 5.2, for all $(h, y, t) \in \mathcal{S}, g_{y}^{h}(t) / \log (t) \geq\left(1-A^{-1}\right) g(t) / \log (t)>1$, so by Lemma 4.10, applying Lemma 5.1 with $H(t)=t^{-n}$, for $n>1$, it follows that uniformly in $(h, y, t) \in \mathcal{S}$,

$$
\begin{aligned}
\mathbb{P}\left(X_{t}^{\left(0, \frac{g_{y}^{h}(t)}{\log (t)}\right)}>K g_{y}^{h}(t)\right) & \stackrel{4.10}{\leq} \frac{\Phi_{y}^{h}(t)}{f(y)-h} \mathbb{P}\left(X_{t}^{\left(0, \frac{g_{y}^{h}(t)}{\log (t)}\right)}>K g_{y}^{h}(t)\right) \\
& \stackrel{5.1}{\leq} \frac{\Phi_{y}^{h}(t)}{f(y)-h} \exp ((*)) t^{-n}, \\
(*) & \lesssim t \log \left(t^{n}\right) t^{n \frac{g_{y}^{h}(t)}{K g_{y}^{h}(t) \log (t)}} \bar{\Pi}\left(\frac{g_{y}^{h}(t)}{\log (t)}\right) \frac{g_{y}^{h}(t)}{K g_{y}^{h}(t) \log (t)} \\
& =\frac{n}{K} e^{\frac{n}{K}} t \bar{\Pi}\left(\frac{g_{y}^{h}(t)}{\log (t)}\right) \lesssim 1,
\end{aligned}
$$

since $\lim _{t \rightarrow \infty} t \bar{\Pi}\left(g_{y}^{h}(t) / \log (t)\right)=0$, uniformly in $h, y$, by Lemma 5.2 and (3.1), and so $\lim _{t \rightarrow \infty}(*)=0$. Thus $(a) \lesssim t^{-n} \Phi_{y}^{h}(t) /(f(y)-h) \leq t^{-1} \log (t)^{-1-\varepsilon} \Phi_{y}^{h}(t) /(f(y)-$ $h)$, uniformly in $(h, y, t) \in \mathcal{S}$, as required for (5.11).

To show $(a) \leq \Phi_{y}^{h}(t) u(t) \bar{\Pi}(g(t)) /(f(y)-h)$, by Lemma 5.1 with $H(t)=\bar{\Pi}(g(t))^{2}$, applying Lemma 5.2 , as $\bar{\Pi}$ is regularly varying at $\infty$ with index $-\alpha \in(-1,0)$, uniformly in $(h, y, t) \in \mathcal{S}$,

$$
\begin{gathered}
\mathbb{P}\left(X_{t}^{\left(0, \frac{g_{y}^{h}(t)}{\log (t)}\right)}>K g_{y}^{h}(t)\right) \stackrel{5.1}{\leq} \exp ((*)) \bar{\Pi}(g(t))^{2}, \\
(*) \lesssim t \log \left(\bar{\Pi}(g(t))^{-2}\right) \bar{\Pi}(g(t))^{-\frac{2}{K \log (t)}} \bar{\Pi}\left(\frac{g_{y}^{h}(t)}{\log (t)}\right) \frac{g_{y}^{h}(t)}{K g_{y}^{h}(t) \log (t)}
\end{gathered}
$$




$$
\begin{aligned}
& \stackrel{5.2}{\lesssim} t \bar{\Pi}(g(t))^{-\frac{2}{K \log (t)}} \frac{\log \left(\bar{\Pi}(g(t))^{-2}\right)}{\log (t)} \bar{\Pi}\left(\frac{g(t)}{\log (t)}\right) \\
& =2 t \frac{\log \left(\frac{1}{\bar{\Pi}(g(t))}\right)}{\log (t)} e^{\frac{2}{K \log (t)} \log \left(\frac{1}{\bar{\Pi}(g(t))}\right)} \bar{\Pi}\left(\frac{g(t)}{\log (t)}\right) .
\end{aligned}
$$

Now, using the inequality $x \leq e^{x}$, bounding $\bar{\Pi}(g(t) / \log (t)) / \bar{\Pi}(g(t)) \leq t$, it follows that

$$
\begin{aligned}
(*) & \lesssim t \bar{\Pi}\left(\frac{g(t)}{\log (t)}\right) e^{\frac{2+K}{K \log (t)} \log \left(\frac{1}{\bar{\Pi}(g(t))}\right)} \\
& =t \bar{\Pi}\left(\frac{g(t)}{\log (t)}\right) e^{\frac{2+K}{K \log (t)} \log \left(\frac{t \bar{\Pi}\left(\frac{g(t)}{\log (t)}\right)}{\bar{\Pi}(g(t)}\right)+\frac{2+K}{K \log (t)} \log \left(\frac{1}{t \bar{\Pi}\left(\frac{g(t)}{\log (t)}\right)}\right)} \\
& \lesssim t \bar{\Pi}\left(\frac{g(t)}{\log (t)}\right) e^{\frac{2+K}{k \log (t)} \log \left(\frac{1}{t \bar{\Pi}\left(\frac{g(t)}{\log (t)}\right)}\right)}=e^{\left[\frac{2+K}{K \log (t)}-1\right] \log \left(\frac{1}{t \bar{\Pi}\left(\frac{g(t)}{\log (t)}\right)}\right)},
\end{aligned}
$$

which is thus bounded uniformly in $(h, y, t) \in \mathcal{S}$ because $\lim _{t \rightarrow \infty} t \bar{\Pi}(g(t) / \log (t))=$ 0 by (3.1). By (5.15) and Lemma 4.10, it follows that $(a) \leq$ $\Phi_{y}^{h}(t) u(t) \bar{\Pi}(g(t)) /(f(y)-h)$ uniformly in $(h, y, t) \in \mathcal{S}$, for suitable $u$, as required for (5.12).

Partitioning $(b)$. Now we partition $(b)$. Let $\Delta_{m}^{(a, b)}$ denote the time of our subordinator's $m$ th jump of size larger in $(a, b)$, as in (3.8). With $\beta$ as in (3.1), for $m \geq 1$ such that $m>\beta /(\beta-1)$ and $m>1 /(\alpha(\beta-1))$, for $c>0$ such that $1-(m-1) c>0$, for all $t$ large enough that $1 / \log (t) \leq c$,

$$
\begin{aligned}
(b) & =\mathbb{P}\left(\mathcal{O}_{t, X^{\left(0, g_{y}^{h}(t)\right)}}^{g_{y}^{h}} ; \Delta_{1}^{\left(\frac{g_{y}^{h}(t)}{\log (t)}, g_{y}^{h}(t)\right)} \leq t\right) \\
& =\mathbb{P}\left(\mathcal{O}_{t, X^{\left(0, g_{y}^{h}(t)\right)}}^{g_{y}^{h}} ; \Delta_{1}^{\left(\frac{g_{y}^{h}(t)}{\log (t)}, c g_{y}^{h}(t)\right)} \leq t ; \Delta_{m}^{\left(\frac{g_{y}^{h}(t)}{\log (t)}, c g_{y}^{h}(t)\right)}>t ; \Delta_{1}^{c g_{y}^{h}(t)}>t\right) \\
& +\mathbb{P}\left(\mathcal{O}_{t, X^{\left(0, g_{y}^{h}(t)\right)}}^{g^{h}} ; \Delta_{m}^{\left(\frac{g_{y}^{h}(t)}{\log (t)}, c g_{y}^{h}(t)\right)} \leq t ; \Delta_{1}^{c g_{y}^{h}(t)}>t\right) \\
& +\mathbb{P}\left(\mathcal{O}_{t, X^{\left(0, g_{y}^{h}(t)\right)}}^{g^{h}} ; \Delta_{1}^{\left(c g_{y}^{h}(t), g_{y}^{h}(t)\right)} \leq t\right) \\
& =:(2 A)+(2 B)+(2 C) .
\end{aligned}
$$

Proof for $(2 A)$. Disintegrating on the value of $\bar{\Delta}_{1}:=\Delta_{1}^{\left(\frac{g_{y}^{h}(t)}{\log (t)}, c g_{y}^{h}(t)\right)}$, the time of the first jump whose size lies between $g_{y}^{h}(t) / \log (t)$ and $c g_{y}^{h}(t)$ as defined in (3.8), which is exponentially distributed,

$$
\begin{aligned}
(2 A) & =\mathbb{P}\left(\mathcal{O}_{t, X^{\left(0, g_{y}^{h}(t)\right)}}^{g^{h}} ; \Delta_{1}^{\left(\frac{g_{y}^{h}(t)}{\log (t)}, c g_{y}^{h}(t)\right)} \leq t ; \Delta_{m}^{\left(\frac{g_{y}^{h}(t)}{\log (t)}, c g_{y}^{h}(t)\right)}>t ; \Delta_{1}^{c g_{y}^{h}(t)}>t\right) \\
& =\int_{0}^{t} \mathbb{P}\left(\mathcal{O}_{t, X^{\left(0, g_{y}^{h}(t)\right)}}^{g_{y}^{h}} ; \Delta_{m}^{\left(\frac{g_{y}^{h}(t)}{\log (t)}, c g_{y}^{h}(t)\right)}>t ; \Delta_{1}^{c g_{y}^{h}(t)}>t \mid \bar{\Delta}_{1}=s\right) \mathbb{P}\left(\bar{\Delta}_{1} \in d s\right)
\end{aligned}
$$




$$
\leq \bar{\Pi}\left(\frac{g_{y}^{h}(t)}{\log (t)}\right) \int_{0}^{t} \mathbb{P}\left(\mathcal{O}_{t, X^{\left(0, c g_{y}^{h}(t)\right)}}^{g_{y}^{h}} ; \Delta_{m}^{\left(\frac{g_{y}^{h}(t)}{\log (t)}, c g_{y}^{h}(t)\right)}>t \mid \bar{\Delta}_{1}=s\right) d s .
$$

Now, with $\bar{\Delta}_{k}$ denoting the time of the $k$ th jump of size between $g_{y}^{h}(t) / \log (t)$ and $c g_{y}^{h}(t)$,

$$
\begin{aligned}
& \mathbb{P}\left(\mathcal{O}_{t, X^{\left(0, c g_{y}^{h}(t)\right)}}^{g_{y}^{h}} ; \Delta_{m}^{\left(\frac{g_{y}^{h}(t)}{\log (t)}, c g_{y}^{h}(t)\right)}>t \mid \bar{\Delta}_{1}=s\right) \\
& =\sum_{k=1}^{m-1} \mathbb{P}\left(\mathcal{O}_{t, X^{\left(0, c g_{y}^{h}(t)\right)}}^{g_{y}^{h}} \mid \bar{\Delta}_{1}=s ; \bar{\Delta}_{k+1}>t ; \bar{\Delta}_{k} \leq t\right) \mathbb{P}\left(\bar{\Delta}_{k+1}>t ; \bar{\Delta}_{k} \leq t \mid \bar{\Delta}_{1}=s\right) \\
& \leq \sum_{k=1}^{m-1} \mathbb{P}\left(\mathcal{O}_{t, X^{\left(0, c g_{y}^{h}(t)\right)}}^{g_{y}^{h}} \mid \bar{\Delta}_{1}=s ; \bar{\Delta}_{k+1}>t ; \bar{\Delta}_{k} \leq t\right),
\end{aligned}
$$

and then it follows that

$$
(2 A) \leq \sum_{k=1}^{m-1} \bar{\Pi}\left(\frac{g_{y}^{h}(t)}{\log (t)}\right) \int_{0}^{t} \mathbb{P}\left(\mathcal{O}_{t, X^{\left(0, c g_{y}^{h}(t)\right)}}^{g^{h}} \mid \bar{\Delta}_{1}=s ; \bar{\Delta}_{k+1}>t ; \bar{\Delta}_{k} \leq t\right) d s .
$$

Now, by (5.4), given that by time $t$ there are $k$ jumps of size in $\left[g_{y}^{h}(t) / \log (t), c g_{y}^{h}(t)\right]$,

$$
\begin{aligned}
(2 A) & \stackrel{(5.4)}{\leq} \sum_{k=1}^{m-1} \bar{\Pi}\left(\frac{g_{y}^{h}(t)}{\log (t)}\right) \int_{0}^{t} \mathbb{P}\left(X_{t}^{\left(0, c g_{y}^{h}(t)\right)}>g_{y}^{h}(t) \mid \bar{\Delta}_{1}=s ; \bar{\Delta}_{k+1}>t ; \bar{\Delta}_{k} \leq t\right) d s \\
& \leq \sum_{k=1}^{m-1} \bar{\Pi}\left(\frac{g_{y}^{h}(t)}{\log (t)}\right) \int_{0}^{t} \mathbb{P}\left(X_{t}^{\left(0, \frac{g_{y}^{h}(t)}{\log (t)}\right)}>(1-k c) g_{y}^{h}(t)\right) d s \\
& =\sum_{k=1}^{m-1} \bar{\Pi}\left(\frac{g_{y}^{h}(t)}{\log (t)}\right) t \mathbb{P}\left(X_{t}^{\left(0, \frac{g_{y}^{h}(t)}{\log (t)}\right)}>(1-k c) g_{y}^{h}(t)\right) \\
& \leq(m-1) \bar{\Pi}\left(\frac{g_{y}^{h}(t)}{\log (t)}\right) t \mathbb{P}\left(X_{t}^{\left(0, \frac{g_{y}^{h}(t)}{\log (t)}\right)}>(1-(m-1) c) g_{y}^{h}(t)\right) .
\end{aligned}
$$

Now, $\lim _{t \rightarrow \infty} t \bar{\Pi}\left(g_{y}^{h}(t) / \log (t)\right) \leq \lim _{t \rightarrow \infty} t \bar{\Pi}((1-1 / A) g(t) / \log (t))=0$ by (3.1), uniformly in $h, y$ by Lemma 5.2. Then by (5.14) and (5.18), it follows that

$$
\begin{aligned}
(2 A) & \lesssim \mathbb{P}\left(X^{\left(0, \frac{g_{y}^{h}(t)}{\log (t)}\right)}>(1-(m-1) c) g_{y}^{h}(t)\right) \stackrel{(5.14)}{\lesssim} \frac{\Phi_{y}^{h}(t)}{f(y)-h} t^{-n} \\
& \lesssim \frac{\Phi_{y}^{h}(t)}{f(y)-h} \frac{1}{t \log (t)^{1+\varepsilon}},
\end{aligned}
$$

uniformly in $(h, y, t) \in \mathcal{S}$, as required for (5.11).

For the remaining bound on $(2 A)$, applying (5.15) and (5.18), recalling $(*) \lesssim 1$ by (5.16), it follows that $(2 A) \leq \bar{\Pi}(g(t))^{2} \leq \Phi_{y}^{h}(t) u(t) \bar{\Pi}(g(t)) /(f(y)-h)$ for suitable $u$, uniformly in $(h, y, t) \in \mathcal{S}$, as required for $(5.12)$. 
Proof for $(2 B)$. Disintegrating on the value of $\Delta_{1}^{\left(\frac{g_{y}^{h}(t)}{\log (t)}, c g_{y}^{h}(t)\right)}$, which is exponentially distributed with parameter $\bar{\Pi}\left(g_{y}^{h}(t) / \log (t)\right)-\bar{\Pi}\left(c g_{y}^{h}(t)\right) \leq \bar{\Pi}\left(g_{y}^{h}(t) / \log (t)\right)$,

$$
\begin{aligned}
&(2 B)=\mathbb{P}\left(\mathcal{O}_{t, X^{\left(0, g_{y}^{h}(t)\right)}}^{g^{h}} ; \Delta_{m}^{\left(\frac{g_{y}^{h}(t)}{\log (t)}, c g_{y}^{h}(t)\right)} \leq t ; \Delta_{1}^{c g_{y}^{h}(t)}>t\right) \\
& \leq \bar{\Pi}\left(\frac{g_{y}^{h}(t)}{\log (t)}\right) \int_{0}^{t} \mathbb{P}\left(\mathcal{O}_{t, X^{\left(0, g_{y}^{h}(t)\right)}}^{g^{h}} ; \Delta_{m}^{\left(\frac{g_{y}^{h}(t)}{\log (t)}, c g_{y}^{h}(t)\right)} \leq t ;\right. \\
&\left.\Delta_{1}^{c g_{y}^{h}(t)}>t \mid \Delta_{1}^{\left(\frac{g_{y}^{h}(t)}{\log (t)}, c g_{y}^{h}(t)\right)}=s\right) d s \\
& \leq \bar{\Pi}\left(\frac{g_{y}^{h}(t)}{\log (t)}\right) \int_{0}^{t} \mathbb{P}\left(\mathcal{O}_{s}^{g_{y}^{h}} ; \Delta_{m}^{\left(\frac{g_{y}^{h}(t)}{\log (t)}, c g_{y}^{h}(t)\right)} \leq t \mid \Delta_{1}^{\left(\frac{g_{y}^{h}(t)}{\log (t)}, c g_{y}^{h}(t)\right)}=s\right) d s .
\end{aligned}
$$

By (5.3), $\mathcal{O}_{s}^{g_{y}^{h}}=\mathcal{O}_{s-}^{g_{y}^{h}}$. Note that given $\Delta_{1}^{\left(\frac{g_{y}^{h}(t)}{\log (t)}, c g_{y}^{h}(t)\right)}=s, \mathcal{O}_{s-}^{g_{y}^{h}}$ is independent of $\Delta_{m}^{\left(\frac{g_{y}^{h}(t)}{\log (t)}, c g_{y}^{h}(t)\right)}$, so

$$
\begin{aligned}
(2 B) & \leq \bar{\Pi}\left(\frac{g_{y}^{h}(t)}{\log (t)}\right) \int_{0}^{t} \mathbb{P}\left(\mathcal{O}_{s}^{g_{y}^{h}}\right) \mathbb{P}\left(\Delta_{m}^{\left(\frac{g_{y}^{h}(t)}{\log (t)}, c g_{y}^{h}(t)\right)} \leq t \mid \Delta_{1}^{\left(\frac{g_{y}^{h}(t)}{\log (t)}, c g_{y}^{h}(t)\right)}=s\right) d s \\
& \leq \bar{\Pi}\left(\frac{g_{y}^{h}(t)}{\log (t)}\right) \mathbb{P}\left(\Delta_{m-1}^{\frac{g_{y}^{h}(t)}{\log (t)}} \leq t\right) \int_{0}^{t} \mathbb{P}\left(\mathcal{O}_{s}^{g_{y}^{h}}\right) d s .
\end{aligned}
$$

Now, since $\Delta_{1}^{\frac{g_{y}^{h}(t)}{\log (t)}}$ is exponentially distributed with parameter $\bar{\Pi}\left(g_{y}^{h}(t) / \log (t)\right)$,

$\mathbb{P}\left(\Delta_{m-1}^{\frac{g_{y}^{h}(t)}{\log (t)}} \leq t\right) \leq \mathbb{P}\left(\Delta_{1}^{\frac{g_{y}^{h}(t)}{\log (t)}} \leq t\right)^{m-1}=\left(1-e^{-t \bar{\Pi}\left(\frac{g_{y}^{h}(t)}{\log (t)}\right)}\right)^{m-1} \leq t^{m-1} \bar{\Pi}\left(\frac{g_{y}^{h}(t)}{\log (t)}\right)^{m-1}$,

so recalling the notation in (3.12), by Lemma 5.2, uniformly in $(h, y, t) \in \mathcal{S}$,

$$
(2 B) \leq \bar{\Pi}\left(\frac{g_{y}^{h}(t)}{\log (t)}\right)^{m} t^{m-1} \Phi_{y}^{h}(t) \stackrel{5.2}{\lesssim} \bar{\Pi}\left(\frac{g(t)}{\log (t)}\right)^{m} t^{m-1} \Phi_{y}^{h}(t) .
$$

Recall $\bar{\Pi}(x)=x^{-\alpha} L(x)$ for $L$ slowly varying at $\infty$, so by Potter's theorem (Bingham et al., 1989, Theorem 1.5.6), for arbitrarily small $\delta>0$, uniformly in $(h, y, t) \in \mathcal{S}$,

$$
\bar{\Pi}\left(\frac{g(t)}{\log (t)}\right)=\log (t)^{\alpha} g(t)^{-\alpha} L(g(t)) \frac{L\left(\frac{g(t)}{\log (t)}\right)}{L(g(t))} \lesssim \log (t)^{\alpha+\delta} \bar{\Pi}(g(t)) .
$$

Similarly, defining $g_{\beta}(t):=g(t) / \log (t)^{\beta}$, for $\beta>(1+\alpha) /\left(2 \alpha+\alpha^{2}\right)$ as in (3.1), uniformly in $(h, y, t) \in \mathcal{S}$,

$$
\bar{\Pi}\left(\frac{g(t)}{\log (t)}\right)=\log (t)^{\alpha} g(t)^{-\alpha} L\left(g_{\beta}(t)\right) \frac{L\left(\frac{g(t)}{\log (t)}\right)}{L\left(g_{\beta}(t)\right)} \lesssim \log (t)^{\alpha(1-\beta)+\delta(\beta-1)} \bar{\Pi}\left(g_{\beta}(t)\right) .
$$


Applying (5.21) to $\bar{\Pi}(g(t))$ and (5.22) to $\bar{\Pi}(g(t))^{m-1}$, then by (3.1), uniformly in $(h, y, t) \in \mathcal{S}$,

$$
\begin{aligned}
(2 B) & \lesssim \log (t)^{m \alpha+m \delta-(m-1) \alpha \beta+\delta(\beta-1)(m-1)} \bar{\Pi}(g(t)) \bar{\Pi}\left(g_{\beta}(t)\right)^{m-1} t^{m-1} \Phi_{y}^{h}(t) \\
& \stackrel{(3.1)}{\leq} \log (t)^{m \alpha+m \delta-(m-1) \alpha \beta+\delta(\beta-1)(m-1)} \bar{\Pi}(g(t)) \Phi_{y}^{h}(t) .
\end{aligned}
$$

Now, for $\beta$ as in (3.1), $m>\beta /(\beta-1)$, so $m \alpha-(m-1) \alpha \beta<0$, choosing $\delta>0$ small enough, we conclude by Lemma 4.10 that for suitable $u,(2 B) \leq$ $u(t) \bar{\Pi}(g(t)) \Phi_{y}^{h}(t) /(f(y)-h)$, uniformly in $(h, y, t) \in \mathcal{S}$, as required for (5.12).

Now we prove $(2 B) \lesssim t^{-1} \log (t)^{-1-\varepsilon} \Phi_{y}^{h}(t) /(f(y)-h)$. With $g_{\beta}(t):=g(t) / \log (t)^{\beta}$, by (5.20) and (5.22), for arbitrarily small $\delta>0$, uniformly in $(h, y, t) \in \mathcal{S}$,

$$
(2 B) \leq \bar{\Pi}\left(\frac{g(t)}{\log (t)}\right)^{m} t^{m-1} \Phi_{y}^{h}(t) \lesssim \log (t)^{m \alpha(1-\beta)+m \delta} \bar{\Pi}\left(g_{\beta}(t)\right)^{m} t^{m-1} \Phi_{y}^{h}(t) .
$$

By (3.1), $\bar{\Pi}\left(g_{\beta}(t)\right) \lesssim t^{-1}$, and so $(2 B) \lesssim t^{-1} \log (t)^{m \alpha(1-\beta)+m \delta} \Phi_{y}^{h}(t)$. Finally, applying Lemma 4.10, our choice of $m$ ensures $m \alpha(1-\beta)<-1$, so choosing $\delta$ small enough, there exists $\varepsilon>0$ such that $(2 B) \lesssim t^{-1} \log (t)^{-1-\varepsilon} \Phi_{y}^{h}(t) /(f(y)-h)$, uniformly in $(h, y, t) \in \mathcal{S}$, as required for (5.11).

Partitioning $(2 C)$. Define $p^{*}(t):=1-\log (t)^{-\gamma}$ for $\gamma:=(1-\alpha) /(2+\alpha)$, and let $\Delta_{2}^{(a, b)}$ denote the time of our subordinator's second jump of size in $(a, b)$. Then we partition:

$$
\begin{aligned}
(2 C) & =\mathbb{P}\left(\mathcal{O}_{t, X^{\left(0, g_{y}^{h}(t)\right)}}^{g^{h}} ; \Delta_{1}^{\left(c g_{y}^{h}(t), g_{y}^{h}(t)\right)} \leq t\right) \\
& =\mathbb{P}\left(\mathcal{O}_{t, X^{\left(0, g_{y}^{h}(t)\right)}}^{g_{y}^{h}} ; \Delta_{1}^{\left(c g_{y}^{h}(t), g_{y}^{h}(t)\right)} \leq t ; \Delta_{1}^{\left(p^{*}(t) g_{y}^{h}(t), g_{y}^{h}(t)\right)} \leq t\right) \\
& +\mathbb{P}\left(\mathcal{O}_{t, X^{\left(0, g_{y}^{h}(t)\right)}}^{g_{y}^{h}} ; \Delta_{2}^{\left(c g_{y}^{h}(t), g_{y}^{h}(t)\right)} \leq t ; \Delta_{1}^{\left(p^{*}(t) g_{y}^{h}(t), g_{y}^{h}(t)\right)}>t\right) \\
& +\mathbb{P}\left(\mathcal{O}_{t, X^{\left(0, g_{y}^{h}(t)\right)}}^{g^{h}} ; \Delta_{1}^{\left(c g_{y}^{h}(t), g_{y}^{h}(t)\right)} \leq t ; \Delta_{2}^{\left(c g_{y}^{h}(t), g_{y}^{h}(t)\right)}>t ; \Delta_{1}^{\left(p^{*}(t) g_{y}^{h}(t), g_{y}^{h}(t)\right)}>t\right) \\
& =:(2 C a)+(2 C b)+(2 C c) .
\end{aligned}
$$

Proof for $(2 C a)$. As $c \in(0,1)$ is fixed, $c<1-\log (t)^{-\gamma}=p^{*}(t)$ for all large enough $t$, and so

$$
(2 C a)=\mathbb{P}\left(\mathcal{O}_{t, X^{\left(0, g_{y}^{h}(t)\right)}}^{g_{y}^{h}} ; \Delta_{1}^{\left(p^{*}(t) g_{y}^{h}(t), g_{y}^{h}(t)\right)} \leq t\right) .
$$

Disintegrating on the value of $\Delta_{1}^{\left(p^{*}(t) g_{y}^{h}(t), g_{y}^{h}(t)\right)}$, then by (5.4) and the independence as in (5.3),

$$
\begin{aligned}
(2 C a) & \leq\left[\bar{\Pi}\left(p^{*}(t) g_{y}^{h}(t)\right)-\bar{\Pi}\left(g_{y}^{h}(t)\right)\right] \int_{0}^{t} \mathbb{P}\left(\mathcal{O}_{t, X^{\left(0, g_{y}^{h}(t)\right)}}^{g_{y}^{h}} \mid \Delta_{1}^{\left(p^{*}(t) g_{y}^{h}(t), g_{y}^{h}(t)\right)}=s\right) d s \\
& \stackrel{(5.3)}{\leq}\left[\bar{\Pi}\left(p^{*}(t) g_{y}^{h}(t)\right)-\bar{\Pi}\left(g_{y}^{h}(t)\right)\right] \int_{0}^{t} \mathbb{P}\left(\begin{array}{c}
\mathcal{O}_{y}^{g_{y}^{h}} \\
s, X^{\left(0, p^{*}(t) g_{y}^{h}(t)\right)}
\end{array}\right) d s \\
& \leq\left[\bar{\Pi}\left(p^{*}(t) g_{y}^{h}(t)\right)-\bar{\Pi}\left(g_{y}^{h}(t)\right)\right] \Phi_{y}^{h}(t)
\end{aligned}
$$




$$
=\bar{\Pi}\left(p^{*}(t) g_{y}^{h}(t)\right)\left(1-\frac{\bar{\Pi}\left(g_{y}^{h}(t)\right)}{\bar{\Pi}\left(p^{*}(t) g_{y}^{h}(t)\right)}\right) \Phi_{y}^{h}(t) .
$$

By Lemma $5.2, \bar{\Pi}\left(p^{*}(t) g_{y}^{h}(t)\right) \lesssim \bar{\Pi}\left(p^{*}(t) g(t)\right) \lesssim \bar{\Pi}(g(t))$ uniformly in $(h, y, t) \in \mathcal{S}$, so

$$
(2 C a) \lesssim \bar{\Pi}(g(t))\left(1-\frac{\bar{\Pi}\left(g_{y}^{h}(t)\right)}{\bar{\Pi}\left(p^{*}(t) g_{y}^{h}(t)\right)}\right) \Phi_{y}^{h}(t) .
$$

As $\lim _{t \rightarrow \infty} p^{*}(t)=1$ and $\bar{\Pi}$ is CRV at $\infty, \lim _{t \rightarrow \infty} \bar{\Pi}\left(p^{*}(t) g_{y}^{h}(t)\right) / \bar{\Pi}\left(g_{y}^{h}(t)\right)=1$. Now, $g_{y}^{h}(t) \gtrsim g(t)$ uniformly in $y, h$ by Lemma 5.2 , so by (5.24) and Lemma 4.10, $(2 C a) \leq \Phi_{y}^{h}(t) u(t) \bar{\Pi}(g(t)) /(f(y)-h)$ for suitable $u$, uniformly in $(h, y, t) \in \mathcal{S}$, as required for (5.12).

Now we prove $(2 C a) \lesssim t^{-1} \log (t)^{-1-\varepsilon} \Phi_{y}^{h}(t) /(f(y)-h)$. As $\bar{\Pi}$ is regularly varying at $\infty, \bar{\Pi}(x)=x^{-\alpha} L(x)$ for $L$ slowly varying at $\infty$, where $x^{N} L(x)$ is non-decreasing. Then by (5.24), uniformly in $(h, y, t) \in \mathcal{S}$,

$$
\begin{aligned}
(2 C a) & \lesssim \bar{\Pi}(g(t))\left(1-\frac{p^{*}(t)^{\alpha} L\left(g_{y}^{h}(t)\right)}{L\left(p^{*}(t) g_{y}^{h}(t)\right)}\right) \Phi_{y}^{h}(t) \\
& =\bar{\Pi}(g(t))\left(1-\frac{p^{*}(t)^{\alpha+N} g_{y}^{h}(t)^{N} L\left(g_{y}^{h}(t)\right)}{p^{*}(t)^{N} g_{y}^{h}(t)^{N} L\left(p^{*}(t) g_{y}^{h}(t)\right)}\right) \Phi_{y}^{h}(t) \\
& \leq \bar{\Pi}(g(t))\left(1-p^{*}(t)^{\alpha+N}\right) \Phi_{y}^{h}(t) \\
& =\bar{\Pi}(g(t))\left(1-\left(1-\log (t)^{-\gamma}\right)^{\alpha+N}\right) \Phi_{y}^{h}(t) \\
& \leq(\alpha+N) \bar{\Pi}(g(t)) \log (t)^{-\gamma} \Phi_{y}^{h}(t) .
\end{aligned}
$$

For $\beta$ as in (3.1), $g_{\beta}(t):=g(t) / \log (t)^{\beta}$, by Potter's theorem and (3.1), for arbitrarily small $\tau>0, \bar{\Pi}(g(t)) \lesssim \bar{\Pi}\left(g_{\beta}(t)\right) \log (t)^{-\alpha \beta+\beta \tau} \leq t^{-1} \log (t)^{-\alpha \beta+\beta \tau}$ uniformly in $(h, y, t) \in \mathcal{S}$, so that

$$
(2 C a) \lesssim t^{-1} \log (t)^{-\gamma-\alpha \beta+\beta \tau} \Phi_{y}^{h}(t) .
$$

Since $\gamma=(1-\alpha) /(2+\alpha)>1-\alpha \beta$, we may choose $\tau$ sufficiently small that $-\gamma-\alpha \beta+\beta \tau<-1-\varepsilon<-1$. Then it follows that $(2 C a) \lesssim t^{-1} \log (t)^{-1-\varepsilon} \Phi_{y}^{h}(t)$, uniformly in $(h, y, t) \in \mathcal{S}$, as required for (5.11).

Proof for (2Cb). Disintegrating on the value of $\hat{\Delta}_{1}^{c}:=\Delta_{1}^{\left(c g_{y}^{h}(t), g_{y}^{h}(t)\right)}$, by (5.3),

$$
\begin{aligned}
(2 C b) \leq & \mathbb{P}\left(\mathcal{O}_{t, X^{\left(0, g_{y}^{h}(t)\right)}}^{g_{y}^{h}} ; \Delta_{2}^{\left(c g_{y}^{h}(t), g_{y}^{h}(t)\right)} \leq t ; \Delta_{1}^{\left(p^{*}(t) g_{y}^{h}(t), g_{y}^{h}(t)\right)}>t\right) \\
\leq & \mathbb{P}\left(\mathcal{O}_{t, X^{\left(0, g_{y}^{h}(t)\right)}}^{g^{h}} ; \Delta_{2}^{\left(c g_{y}^{h}(t), g_{y}^{h}(t)\right)} \leq t\right) \\
\leq & \bar{\Pi}\left(c g_{y}^{h}(t)\right) \int_{0}^{t} \mathbb{P}\left(\mathcal{O}_{t, X^{\left(0, g_{y}^{h}(t)\right)}}^{g^{h}} ; \Delta_{2}^{\left(c g_{y}^{h}(t), g_{y}^{h}(t)\right)} \leq t \mid \hat{\Delta}_{1}^{c}=s\right) d s \\
\leq & \bar{\Pi}\left(c g_{y}^{h}(t)\right) \int_{0}^{t} \mathbb{P}\left(\mathcal{O}_{s, X^{\left(0, g_{y}^{h}(t)\right)}}^{g^{h}} \mid \hat{\Delta}_{1}^{c}=s\right) \\
& \times \mathbb{P}\left(\Delta_{2}^{\left(c g_{y}^{h}(t), g_{y}^{h}(t)\right)} \leq t \mid \hat{\Delta}_{1}^{c}=s\right) d s
\end{aligned}
$$




$$
\stackrel{(5.3)}{\leq} \bar{\Pi}\left(c g_{y}^{h}(t)\right) \mathbb{P}\left(\Delta_{1}^{c g_{y}^{h}(t)} \leq t\right) \int_{0}^{t} \mathbb{P}\left(\mathcal{O}_{s}^{g_{y}^{h}}\right) d s
$$

Recall for $L$ slowly varying at $\infty, \bar{\Pi}(x)=x^{-\alpha} L(x)$. Now, $\mathbb{P}\left(\Delta_{1}^{c g_{y}^{h}(t)} \leq t\right)=1-$ $e^{-t \bar{\Pi}\left(c g_{y}^{h}(t)\right)} \leq t \bar{\Pi}\left(c g_{y}^{h}(t)\right)$, so by (3.12) and Lemma 5.2, uniformly in $(h, y, t) \in \mathcal{S}$,

$$
\begin{aligned}
(2 C b) & \stackrel{(3.12)}{\leq} \bar{\Pi}\left(c g_{y}^{h}(t)\right)^{2} t \Phi_{y}^{h}(t) \stackrel{5.2}{\lesssim} \bar{\Pi}(g(t))^{2} t \Phi_{y}^{h}(t)=g(t)^{-2 \alpha} L(g(t))^{2} t \Phi_{y}^{h}(t) \\
& =\log (t)^{-2 \alpha \beta}\left(\frac{g(t)}{\log (t)^{\beta}}\right)^{-2 \alpha} L\left(\frac{g(t)}{\log (t)^{\beta}}\right)^{2} \frac{L(g(t))^{2}}{L\left(\frac{g(t)}{\log (t)^{\beta}}\right)^{2}} t \Phi_{y}^{h}(t) \\
& =\log (t)^{-2 \alpha \beta} \bar{\Pi}\left(\frac{g(t)}{\log (t)^{\beta}}\right)^{2} \frac{L(g(t))^{2}}{L\left(\frac{g(t)}{\log (t)^{\beta}}\right)^{2}} t \Phi_{y}^{h}(t) .
\end{aligned}
$$

By Potter's theorem (Bingham et al., 1989, Theorem 1.5.6), for arbitrarily small $\delta>0$, uniformly in $t$,

$$
(2 C b) \lesssim \log (t)^{-2 \alpha \beta} \bar{\Pi}\left(\frac{g(t)}{\log (t)^{\beta}}\right)^{2} \log (t)^{2 \beta \delta} t \Phi_{y}^{h}(t) .
$$

It follows by (3.1) that $\bar{\Pi}\left(g(t) / \log (t)^{\beta}\right)^{2} \lesssim t^{-2}$ uniformly in $t$, and hence

$$
(2 C b) \lesssim \log (t)^{-2 \alpha \beta} \log (t)^{2 \beta \delta} t^{-1} \Phi_{y}^{h}(t) .
$$

Now, $2 \alpha \beta>1$ by (3.1). Taking $\delta$ small enough that $2 \alpha \beta-2 \beta \delta \geq 1+\varepsilon>1$, it follows by Lemma 4.10 that $(2 C b) \lesssim t^{-1} \log (t)^{-1-\varepsilon} \Phi_{y}^{h}(t) /(f(y)-h)$, uniformly in $(h, y, t) \in \mathcal{S}$, as required for (5.11).

Now we show $(2 C b) \leq \Phi_{y}^{h}(t) u(t) \bar{\Pi}(g(t)) /(f(y)-h)$. By (5.25), as $\lim _{t \rightarrow \infty} t \bar{\Pi}(g(t))=$ 0 by (3.1), uniformly in $(h, y, t) \in \mathcal{S}$,

$$
(2 C b) \lesssim \bar{\Pi}(g(t))^{2} t \Phi_{y}^{h}(t)=o(1) \times \bar{\Pi}(g(t)) \Phi_{y}^{h}(t),
$$

so by Lemma 4.10, $(2 C b) \leq \Phi_{y}^{h}(t) u(t) \bar{\Pi}(g(t)) /(f(y)-h)$ for suitable $u$, uniformly in $(h, y, t) \in \mathcal{S}$, as required for (5.12).

Partitioning $(2 C c)$. Disintegrating on the value of $\Delta_{1}^{c, p^{*}}:=\Delta_{1}^{\left(c g_{y}^{h}(t), p^{*}(t) g_{y}^{h}(t)\right)}$, by (5.3), (5.4), and Lemma 5.2, uniformly in $(h, y, t) \in \mathcal{S}$,

$$
\begin{gathered}
(2 C c)=\mathbb{P}\left(\mathcal{O}_{t, X^{\left(0, g_{y}^{h}(t)\right)}}^{g_{y}^{h}} ; \Delta_{1}^{\left(c g_{y}^{h}(t), g_{y}^{h}(t)\right)} \leq t ; \Delta_{2}^{\left(c g_{y}^{h}(t), g_{y}^{h}(t)\right)}>t ; \Delta_{1}^{\left(p^{*}(t) g_{y}^{h}(t), g_{y}^{h}(t)\right)}>t\right) \\
\leq \bar{\Pi}\left(c g_{y}^{h}(t)\right) \int_{0}^{t} \mathbb{P}\left(\mathcal{O}_{t, X_{y}^{\left(0, g_{y}^{h}(t)\right)}}^{g^{h}} ;\right. \\
\left.\Delta_{2}^{\left(c g_{y}^{h}(t), g_{y}^{h}(t)\right)}>t ; \Delta_{1}^{\left(p^{*}(t) g_{y}^{h}(t), g_{y}^{h}(t)\right)}>t \mid \Delta_{1}^{c, p^{*}}=s\right) d s \\
\stackrel{(5.4)}{\leq} \bar{\Pi}\left(c g_{y}^{h}(t)\right) \int_{0}^{t} \mathbb{P}\left(\mathcal{O}_{s, X_{y}^{\left(0, c g_{y}^{h}(t)\right)}}^{g^{h}} ;\right. \\
\left.\hat{X}_{t-s}^{\left(0, c g_{y}^{h}(t)\right)}+X_{s-}^{\left(0, c g_{y}^{h}(t)\right)}>\left(1-p^{*}(t)\right) g_{y}^{h}(t) \mid \Delta_{1}^{c, p^{*}}=s\right) d s
\end{gathered}
$$




$$
\begin{gathered}
\stackrel{(5.3)}{=} \bar{\Pi}\left(c g_{y}^{h}(t)\right) \int_{0}^{t} \mathbb{P}\left(\mathcal{O}_{s, X^{\left(0, c g_{y}^{h}(t)\right)}}^{g_{g}^{h}} ;\right. \\
\left.\hat{X}_{t-s}^{\left(0, c g_{y}^{h}(t)\right)}+X_{s-}^{\left(0, c g_{y}^{h}(t)\right)}>\left(1-p^{*}(t)\right) g_{y}^{h}(t)\right) d s \\
\stackrel{5.2}{\lesssim} \bar{\Pi}(g(t)) \int_{0}^{t} \mathbb{P}\left(\mathcal{O}_{s, X^{\left(0, c g_{y}^{h}(t)\right)}}^{g_{y}^{h}} ; \hat{X}_{t-s}^{\left(0, c g_{y}^{h}(t)\right)}+X_{s-}^{\left(0, c g_{y}^{h}(t)\right)}>\left(1-p^{*}(t)\right) g_{y}^{h}(t)\right) d s,
\end{gathered}
$$

where $\hat{X}$ is an independent copy of $X$, and we use that the jump at time $s$ has size at most $p^{*}(t) g_{y}^{h}(t)$. Recall that $1-p^{*}(t)=\log (t)^{-\gamma}$. Then partitioning according to $\left\{X_{s-}^{\left(0, c g_{y}^{h}(t)\right)}>g_{y}^{h}(t) /\left(2 \log (t)^{\gamma}\right)\right\}$,

$$
\begin{aligned}
(2 C c) & \lesssim \bar{\Pi}(g(t)) \int_{0}^{t} \mathbb{P}\left(\mathcal{O}_{s, X^{\left(0, c g_{y}^{h}(t)\right)}}^{g_{y}^{h}} ; \hat{X}_{t-s}^{\left(0, c g_{y}^{h}(t)\right)}>\frac{g_{y}^{h}(t)}{2 \log (t)^{\gamma}}\right) d s \\
& +\bar{\Pi}(g(t)) \int_{0}^{t} \mathbb{P}\left(\mathcal{O}_{s, X^{\left(0, c g_{y}^{h}(t)\right)}}^{g^{h}} ; X_{s-}^{\left(0, c g_{y}^{h}(t)\right)}>\frac{g_{y}^{h}(t)}{2 \log (t)^{\gamma}}\right) d s \\
& =:(S)+\left(S^{*}\right) .
\end{aligned}
$$

Next we will bound $(S)$, then later we will split up $\left(S^{*}\right)$ into more pieces.

Proof for $(S)$. As $\hat{X}$ is an independent copy of $X$, we can write

$$
\begin{aligned}
(S) & =\bar{\Pi}(g(t)) \int_{0}^{t} \mathbb{P}\left(\mathcal{O}_{s, X^{\left(0, c g_{y}^{h}(t)\right)}}^{g_{h}^{h}}\right) \mathbb{P}\left(X_{t-s}^{\left(0, c g_{y}^{h}(t)\right)}>\frac{g_{y}^{h}(t)}{2 \log (t)^{\gamma}}\right) d s \\
& \leq \bar{\Pi}(g(t)) \mathbb{P}\left(X_{t}^{\left(0, c g_{y}^{h}(t)\right)}>\frac{g_{y}^{h}(t)}{2 \log (t)^{\gamma}}\right) \Phi_{y}^{h}(t) .
\end{aligned}
$$

Since $\bar{\Pi}$ is regularly varying at $\infty$, applying Potter's theorem (Bingham et al., 1989, Theorem 1.5.6), with $\beta$ as in (3.1), to $\bar{\Pi}(g(t)) / \bar{\Pi}\left(g(t) / \log (t)^{\beta}\right)$, for arbitrarily small $\tau>0$, it follows by (3.1) that uniformly in $(h, y, t) \in \mathcal{S}$,

$$
\begin{aligned}
(S) & \lesssim \bar{\Pi}\left(\frac{g(t)}{\log (t)^{\beta}}\right) \log (t)^{-\alpha \beta+\tau \beta} \mathbb{P}\left(X_{t}^{\left(0, c g_{y}^{h}(t)\right)}>\frac{g_{y}^{h}(t)}{2 \log (t)^{\gamma}}\right) \Phi_{y}^{h}(t) \\
& \lesssim t^{-1} \log (t)^{-\alpha \beta+\tau \beta} \mathbb{P}\left(X_{t}^{\left(0, c g_{y}^{h}(t)\right)}>\frac{g_{y}^{h}(t)}{2 \log (t)^{\gamma}}\right) \Phi_{y}^{h}(t) .
\end{aligned}
$$

Now, we will show that there exists $\varepsilon>0$ such that uniformly in $(h, y, t) \in \mathcal{S}$,

$$
\mathbb{P}\left(X_{t}^{\left(0, c g_{y}^{h}(t)\right)}>\frac{g_{y}^{h}(t)}{2 \log (t)^{\gamma}}\right) \lesssim \log (t)^{-1-\varepsilon+\alpha \beta-\tau \beta} .
$$

Then it follows from (5.29), (5.30), and Lemma 4.10 that

$$
(S) \lesssim \frac{\Phi_{y}^{h}(t)}{t \log (t)^{1+\alpha \beta}} \leq \frac{\Phi_{y}^{h}(t)}{t \log (t)^{1+\varepsilon}} \leq \frac{\Phi_{y}^{h}(t)}{t \log (t)^{1+\varepsilon}}\left(1+\frac{1}{f(y)-h}\right),
$$

uniformly in $(h, y, t) \in \mathcal{S}$, as required for (5.11). Moreover, it follows by (5.27) and (5.30) that for suitable $u,(S) \leq \Phi_{y}^{h}(t) u(t) \bar{\Pi}(g(t)) /(f(y)-h)$, uniformly in $(h, y, t) \in \mathcal{S}$, as required for $(5.12)$. 
Now, to prove (5.30), we set $M:=\gamma+2 /(2+\alpha)=(3-\alpha) /(2+\alpha)$, and partition as follows

$$
\begin{aligned}
& \mathbb{P}\left(X_{t}^{\left(0, c g_{y}^{h}(t)\right)}>g_{y}^{h}(t) / 2 \log (t)^{\gamma}\right) \\
& =\mathbb{P}\left(X_{t}^{\left(0, c g_{y}^{h}(t)\right)}>\frac{g_{y}^{h}(t)}{2 \log (t)^{\gamma}} ; \Delta_{1}^{\frac{g_{y}^{h}(t)}{\log (t)^{M}}} \leq t\right)+\mathbb{P}\left(X_{t}^{\left(0, c g_{y}^{h}(t)\right)}>\frac{g_{y}^{h}(t)}{2 \log (t)^{\gamma}} ; \Delta_{1}^{\frac{g_{y}^{h}(t)}{\log (t)^{M}}}>t\right) \\
& =:(Q)+\left(Q^{\prime}\right) .
\end{aligned}
$$

Then as $\Delta_{1}^{\frac{g_{y}^{h}(t)}{\log (t)^{M}}}$ is exponentially distributed with rate $\bar{\Pi}\left(g_{y}^{h}(t) / \log (t)^{M}\right)$, we can bound

$$
(Q) \leq \mathbb{P}\left(\Delta_{1}^{\frac{g_{y}^{h}(t)}{\log (t)^{M}}} \leq t\right) \leq 1-e^{-t \bar{\Pi}\left(g_{y}^{h}(t) / \log (t)^{M}\right)} \leq t \bar{\Pi}\left(\frac{g_{y}^{h}(t)}{\log (t)^{M}}\right) .
$$

By Lemma 5.2 and (3.1), it follows for arbitrarily small $\kappa>0$, uniformly in $(h, y, t) \in \mathcal{S}$, applying Potter's Theorem to $\bar{\Pi}\left(g(t) / \log (t)^{M}\right) / \bar{\Pi}\left(g(t) / \log (t)^{\beta}\right)$, that

$$
\begin{aligned}
(Q) & \stackrel{5.2}{\lesssim} t \bar{\Pi}\left(\frac{g(t)}{\log (t)^{M}}\right) \lesssim t \bar{\Pi}\left(\frac{g(t)}{\log (t)^{\beta}}\right) \log (t)^{-\alpha(\beta-M)+\kappa(\beta-M)} \\
& \stackrel{(3.1)}{\lesssim} \log (t)^{-\alpha(\beta-M)+\kappa(\beta-M)},
\end{aligned}
$$

and then in order for (5.30) to hold, we need $-\alpha(\beta-M)+\kappa(\beta-M) \leq-1-\varepsilon+$ $\alpha \beta-\tau \beta$, so taking $\kappa, \tau, \varepsilon$ small enough, we need $-\alpha(\beta-M)<-1+\alpha \beta$, that is, $(3-\alpha) /(2+\alpha)=M<2 \beta-1 / \alpha$. This is indeed true since $\beta>(1+2 \alpha) /\left(2 \alpha+\alpha^{2}\right)$, from which it follows that

$$
2 \beta-\frac{1}{\alpha}>2 \frac{1+2 \alpha}{2 \alpha+\alpha^{2}}-\frac{1}{\alpha}=\frac{2+4 \alpha-2-\alpha}{2 \alpha+\alpha^{2}}=\frac{3}{2+\alpha}>\frac{3-\alpha}{2+\alpha}=M
$$

and the desired bound for $(Q)$ holds. To bound $\left(Q^{\prime}\right)$, we provide only a proof for $\alpha \beta \leq 1$, as a similar, simpler argument works when $\alpha \beta>1$. By Lemma 5.1 with $H(t)=\log (t)^{-1-\varepsilon+\alpha \beta-\tau \beta}$,

$$
\begin{gathered}
\left(Q^{\prime}\right) \leq \mathbb{P}\left(X_{t}^{\left(0, \frac{g_{y}^{h}(t)}{\log (t)^{M}}\right)}>\frac{g_{y}^{h}(t)}{2 \log (t)^{\gamma}}\right) \stackrel{5.1}{\leq} \exp ((*)) \log (t)^{-1-\varepsilon+\alpha \beta-\tau \beta} \\
(*) \lesssim t \log \left(\log (t)^{1+\varepsilon-\alpha \beta+\tau \beta}\right) \log (t)^{[1+\varepsilon-\alpha \beta+\tau \beta] 2 \log (t)^{\gamma-M}} \bar{\Pi}\left(\frac{g_{y}^{h}(t)}{\log (t)^{M}}\right) 2 \log (t)^{\gamma-M} .
\end{gathered}
$$

Now, for $\eta>0$ small enough that $\eta<M-\gamma$, observe that since $M>\gamma$, uniformly in $t>t_{0}(y)$,

$$
(*) \lesssim t \log (t)^{\frac{\eta}{2}} \log (t)^{\frac{\eta}{2}} \bar{\Pi}\left(\frac{g_{y}^{h}(t)}{\log (t)^{M}}\right) \log (t)^{\gamma-M} \leq t \bar{\Pi}\left(\frac{g_{y}^{h}(t)}{\log (t)^{M}}\right) .
$$

Now, recall $M=(3-\alpha) /(2+\alpha), \beta>(1+2 \alpha) /\left(2 \alpha+\alpha^{2}\right)$, and $\alpha<1$, from which one can verify that $M<\beta$. Then it follows by Lemma 5.2 and (3.1) that uniformly in $(h, y, t) \in \mathcal{S}$,

$$
(*) \stackrel{5.2}{\lesssim} t \bar{\Pi}\left(\frac{g(t)}{\log (t)^{M}}\right) \leq t \bar{\Pi}\left(\frac{g(t)}{\log (t)^{\beta}}\right) \stackrel{(3.1)}{=} o(1)
$$

so the desired bounds for $(Q)$ and $\left(Q^{\prime}\right)$ are proven, and the proof of (5.30) is complete. 
Partitioning $\left(S^{*}\right)$. Now we partition $\left(S^{*}\right)$. For $\beta$ as in $(3.1), \gamma=(1-\alpha) /(2+\alpha)$, and $\delta:=1+\gamma$, write $g_{\delta}(t):=g(t) / \log (t)^{\delta}$. Recall the notation (3.7). Then

$$
\begin{aligned}
& \left(S^{*}\right)=\bar{\Pi}(g(t)) \int_{0}^{t} \mathbb{P}\left(\mathcal{O}_{s, X^{\left(0, c g_{y}^{h}(t)\right)}}^{g_{y}^{h}} ; X_{s-}^{\left(0, c g_{y}^{h}(t)\right)}>\frac{g_{y}^{h}(t)}{2 \log (t)^{\gamma}}\right) d s \\
& =\bar{\Pi}(g(t)) \int_{0}^{t} \mathbb{P}\left(\mathcal{O}_{s, X^{\left(0, c g_{y}^{h}(t)\right)}}^{g_{y}^{h}} ; X_{s-}^{\left(0, c g_{y}^{h}(t)\right)}>\frac{g_{y}^{h}(t)}{2 \log (t)^{\gamma}} ; \Delta_{1}^{g_{\delta}(t)} \leq s\right) d s \\
& +\bar{\Pi}(g(t)) \int_{0}^{t} \mathbb{P}\left(\mathcal{O}_{s, X^{\left(0, c g_{y}^{h}(t)\right)}}^{g_{y}^{h}} ; X_{s-}^{\left(0, c g_{y}^{h}(t)\right)}>\frac{g_{y}^{h}(t)}{2 \log (t)^{\gamma}} ; \Delta_{1}^{g_{\delta}(t)}>s\right) d s \\
& =:\left(S_{1}^{*}\right)+\left(S_{2}^{*}\right) \text {. }
\end{aligned}
$$

Proof for $\left(S_{1}^{*}\right)$. Disintegrating on the value of $\Delta_{1}^{g_{\delta}(t)}$, by (5.3) and (3.12),

$$
\begin{aligned}
&\left(S_{1}^{*}\right) \leq \bar{\Pi}(g(t)) \bar{\Pi}\left(g_{\delta}(t)\right) \int_{0}^{t} \int_{0}^{s} \mathbb{P}\left(\mathcal{O}_{s, X^{\left(0, c g_{y}^{h}(t)\right)}}^{g^{h}} ;\right.\left.X_{s-}^{\left(0, c g_{y}^{h}(t)\right)}>\frac{g_{y}^{h}(t)}{2 \log (t)^{\gamma}} \mid \Delta_{1}^{g_{\delta}(t)}=v\right) d v d s \\
& \leq \bar{\Pi}(g(t)) \bar{\Pi}\left(g_{\delta}(t)\right) \int_{0}^{t} \int_{0}^{t} \mathbb{P}\left(\mathcal{O}_{v, X^{\left(0, g_{\delta}(t)\right)}}^{g^{h}} ;\right. \\
&\left.X_{t}^{\left(0, c g_{y}^{h}(t)\right)}>\frac{g_{y}^{h}(t)}{2 \log (t)^{\gamma}} \mid \Delta_{1}^{g_{\delta}(t)}=v\right) d v d s \\
& \stackrel{(5.3)}{\leq} t \bar{\Pi}(g(t)) \bar{\Pi}\left(g_{\delta}(t)\right) \int_{0}^{t} \mathbb{P}\left(\mathcal{O}_{v, X^{\left(0, g_{\delta}(t)\right)}}^{g_{y}^{h}}\right) d v \stackrel{(3.12)}{\leq} t \bar{\Pi}(g(t)) \bar{\Pi}\left(g_{\delta}(t)\right) \Phi_{y}^{h}(t) .
\end{aligned}
$$

As $\bar{\Pi}$ is regularly varying with index $-\alpha$, for $g_{\beta}(t):=g(t) / \log (t)^{\beta}$ and $\beta$ as in (3.1), applying Potter's theorem (Bingham et al., 1989, Theorem 1.5.6) to $\bar{\Pi}(g(t)) / \bar{\Pi}\left(g_{\beta}(t)\right)$ and $\bar{\Pi}\left(g_{\delta}(t)\right) / \bar{\Pi}\left(g_{\beta}(t)\right)$, for $\tau>0$, uniformly in $t$,

$$
\left(S_{1}^{*}\right) \lesssim t \bar{\Pi}\left(g_{\beta}(t)\right)^{2} \log (t)^{-\alpha \beta-\alpha(\beta-\delta)+\beta \tau+(\beta-\delta) \tau} \Phi_{y}^{h}(t),
$$

Now, by (3.1), $\lim _{t \rightarrow \infty} t \bar{\Pi}\left(g_{\beta}(t)\right)=0$, so uniformly in $t$,

$$
\left(S_{1}^{*}\right) \leq t^{-1} \log (t)^{-\alpha \beta-\alpha(\beta-\delta)+\beta \tau+(\beta-\delta) \tau} \Phi_{y}^{h}(t) .
$$

Now, one can verify that $-\alpha \beta-\alpha(\beta-\delta)<-1$, using that $\delta=1+\gamma=1+(1-$ $\alpha) /(2+\alpha)$ and $\beta>(1+2 \alpha) /\left(2 \alpha+\alpha^{2}\right)$. So taking $\tau$ small enough, we conclude by Lemma 4.10 that $\left(S_{1}^{*}\right) \lesssim t^{-1} \log (t)^{-1-\varepsilon} \Phi_{y}^{h}(t) /(f(y)-h)$, uniformly in $(h, y, t) \in \mathcal{S}$, as required for (5.11).

To show $\left(S_{1}^{*}\right) \leq \Phi_{y}^{h}(t) u(t) \bar{\Pi}(g(t)) /(f(y)-h)$, one can verify that for each $\alpha \in$ $(0,1)$ and for $\beta$ as in $(3.1), \delta=1+(1-\alpha) /(2+\alpha)<(1+2 \alpha) /\left(2 \alpha+\alpha^{2}\right)<\beta$. Thus $\lim _{t \rightarrow \infty} t \bar{\Pi}\left(g_{\delta}(t)\right)=0$ by (3.1). Then by (5.34) and Lemma 4.10 , for suitable $u$, uniformly in $(h, y, t) \in \mathcal{S},\left(S_{1}^{*}\right) \lesssim t \bar{\Pi}(g(t)) \bar{\Pi}\left(g_{\delta}(t)\right) \Phi_{y}^{h}(t) \leq u(t) \bar{\Pi}(g(t)) \Phi_{y}^{h}(t) /(f(y)-$ $h)$, as required for (5.12). 
Proof for $\left(S_{2}^{*}\right)$. Note for $g_{\delta}(t):=g(t) / \log (t)^{\delta}$, by Lemma 5.2, for all $h>0, y>g(h)$, and for all large enough $t, g_{\delta}(t) \leq c g_{y}^{h}(t)$, so

$$
\begin{aligned}
\left(S_{2}^{*}\right) & =\bar{\Pi}(g(t)) \int_{0}^{t} \mathbb{P}\left(\mathcal{O}_{s, X^{\left(0, c g_{y}^{h}(t)\right)}}^{g^{h}} ; X_{s-}^{\left(0, c g_{y}^{h}(t)\right)}>\frac{g_{y}^{h}(t)}{2 \log (t)^{\gamma}} ; \Delta_{1}^{g_{\delta}(t)}>s\right) d s \\
& =\bar{\Pi}(g(t)) \int_{0}^{t} \mathbb{P}\left(\mathcal{O}_{s, X^{\left(0, g_{\delta}(t)\right)}}^{g_{y}^{h}} ; X_{s-}^{\left(0, g_{\delta}(t)\right)}>\frac{g_{y}^{h}(t)}{2 \log (t)^{\gamma}} ; \Delta_{1}^{g_{\delta}(t)}>s\right) d s \\
& \leq \bar{\Pi}(g(t)) \int_{0}^{t} \mathbb{P}\left(X_{s-}^{\left(0, g_{\delta}(t)\right)}>\frac{g_{y}^{h}(t)}{2 \log (t)^{\gamma}}\right) d s \\
& \leq t \bar{\Pi}(g(t)) \mathbb{P}\left(X_{t}^{\left(0, g_{\delta}(t)\right)}>\frac{g_{y}^{h}(t)}{2 \log (t)^{\gamma}}\right) .
\end{aligned}
$$

For $g_{\beta}(t):=g(t) / \log (t)^{\beta}$, with $\beta$ as in (3.1), applying Potter's theorem (Bingham et al., 1989, Theorem 1.5.6) to $\bar{\Pi}(g(t))$, for arbitrarily small $\tau>0$, by Lemma 5.2,

$$
\begin{aligned}
\left(S_{2}^{*}\right) & \lesssim t \bar{\Pi}\left(g_{\beta}(t)\right) \log (t)^{-\alpha \beta+\tau \beta} \mathbb{P}\left(X_{t}^{\left(0, g_{\delta}(t)\right)}>\frac{g_{y}^{h}(t)}{2 \log (t)^{\gamma}}\right) \\
& \quad 5.2 t \bar{\Pi}\left(g_{\beta}(t)\right) \log (t)^{-\alpha \beta+\tau \beta} \mathbb{P}\left(X_{t}^{\left(0, g_{\delta}(t)\right)}>\frac{\left(1-A^{-1}\right) g(t)}{2 \log (t)^{\gamma}}\right) .
\end{aligned}
$$

Applying Lemma 5.1 with $H(t)=1 /\left(t \log (t)^{1+\varepsilon-\alpha \beta}\right), \varepsilon>\tau \beta$, then applying (3.1) and Lemma 4.10, uniformly in $(h, y, t) \in \mathcal{S}$ by Lemma 5.2 ,

$$
\begin{aligned}
\left(S_{2}^{*}\right) & \stackrel{5.1}{\lesssim} t \bar{\Pi}\left(g_{\beta}(t)\right) \log (t)^{-\alpha \beta+\tau \beta} \exp ((*)) \frac{1}{t \log (t)^{1+\varepsilon-\alpha \beta}} \\
& \stackrel{(3.1)}{=} o(1) \times \exp ((*)) \frac{1}{t \log (t)^{1+\varepsilon-\tau \beta}} \\
& \stackrel{4.10}{\leq} o(1) \times \exp ((*)) \frac{1}{t \log (t)^{1+\varepsilon-\tau \beta}} \frac{\Phi_{y}^{h}(t)}{f(y)-h}, \\
(*) & \lesssim t \log \left(t \log (t)^{1+\varepsilon-\alpha \beta}\right)\left(t \log (t)^{1+\varepsilon-\alpha \beta}\right)^{\frac{2 \log (t)^{\gamma-\delta}}{1-A^{-1}}} \bar{\Pi}\left(g_{\delta}(t)\right) \log (t)^{\gamma-\delta} .
\end{aligned}
$$

Now, if $\lim _{t \rightarrow \infty}(*)=0$, then $\left(S_{2}^{*}\right) \lesssim t^{-1} \log (t)^{-1-\varepsilon} \Phi_{y}^{h}(t) /(f(y)-h)$. Indeed,

$$
\begin{aligned}
(*) & \lesssim t \log \left(t \log (t)^{1+\varepsilon-\alpha \beta}\right)\left(t \log (t)^{1+\varepsilon-\alpha \beta}\right)^{\frac{2 \log (t) \gamma-\delta}{1-A^{-1}}} \bar{\Pi}\left(g_{\delta}(t)\right) \log (t)^{\gamma-\delta} \\
& =t \bar{\Pi}\left(g_{\delta}(t)\right) t^{\frac{2 \log (t) \gamma-\delta}{1-A^{-1}}} \log \left(t \log (t)^{1+\varepsilon-\alpha \beta}\right) \log (t)^{(1+\varepsilon-\alpha \beta) \frac{2 \log (t)^{\gamma-\delta}}{1-A^{-1}}+\gamma-\delta} .
\end{aligned}
$$

Now, $\delta>\gamma$, so $\lim _{t \rightarrow \infty} \log (t)^{\gamma-\delta}=0$, and for arbitrarily small $\kappa>0$, uniformly in $t$

$$
(*) \lesssim t \bar{\Pi}\left(g_{\delta}(t)\right) t^{\frac{2 \log (t) \gamma-\delta}{1-A-1}} \log \left(t \log (t)^{1+\varepsilon-\alpha \beta}\right) \log (t)^{(1+\varepsilon-\alpha \beta) \kappa+\gamma-\delta} .
$$


Note that $\log \left(t \log (t)^{1+\varepsilon-\alpha \beta}\right) \lesssim \log (t)$, uniformly in $t$. Then applying Potter's theorem (Bingham et al., 1989, Theorem 1.5.6) to $\bar{\Pi}\left(g_{\delta}(t)\right.$ ), for $\beta$ as in (3.1) and arbitrarily small $c>0$, uniformly in $t$,

$$
(*) \lesssim t \bar{\Pi}\left(g_{\beta}(t)\right) t^{\frac{2 \log (t) \gamma-\delta}{1-A^{-1}}} \log (t)^{1+(1+\varepsilon-\alpha \beta) \kappa-(\beta-\delta) \alpha+(\beta-\delta) c+\gamma-\delta} .
$$

Recalling $\gamma-\delta=-1, t^{\frac{2 \log (t) \gamma-\delta}{1-A^{-1}}}=e^{\frac{2 \log (t)^{1+\gamma-\delta}}{1-A^{-1}}}=e^{\frac{2}{1-A^{-1}}}$. As $\lim _{t \rightarrow \infty} t \bar{\Pi}\left(g_{\beta}(t)\right)=0$ by (3.1), using that $1+\gamma-\delta=0$, it follows that uniformly in $t$,

$$
(*) \lesssim \log (t)^{1+(1+\varepsilon-\alpha \beta) \kappa-(\beta-\delta) \alpha+(\beta-\delta) c+\gamma-\delta}=\log (t)^{(1+\varepsilon-\alpha \beta) \kappa-(\beta-\delta) \alpha+(\beta-\delta) c} .
$$

Now, $\delta<\beta$, so $-(\beta-\delta) \alpha<0$. Choosing $\kappa, c$ small enough that the exponent in (5.37) is negative, $\lim _{t \rightarrow \infty}(*)=0$. Then by (5.36), uniformly in $(h, y, t) \in \mathcal{S}$, $\left(S_{2}^{*}\right) \lesssim t^{-1} \log (t)^{-1-\varepsilon} \Phi_{y}^{h}(t) /(f(y)-h)$, as required for (5.11).

To prove $\left(S_{2}^{*}\right) \leq \Phi_{y}^{h}(t) u(t) \bar{\Pi}(g(t)) /(f(y)-h)$, applying Lemma 5.2 and Lemma 5.1 with $H(t)=1 /(t \log (\log (t)))$ to $(5.35)$, uniformly in $(h, y, t) \in \mathcal{S}$,

$$
\begin{gathered}
\left(S_{2}^{*}\right) \stackrel{(5.35)}{\lesssim} t \bar{\Pi}(g(t)) \mathbb{P}\left(X_{t}^{\left(0, g_{\delta}(t)\right)}>\frac{\left(1-A^{-1}\right) g(t)}{2 \log (t)^{\gamma}}\right) \stackrel{5.1}{\leq} \frac{\bar{\Pi}(g(t))}{\log (\log (t))} \exp ((*)), \\
(*) \lesssim t \log (t \log (\log (t)))(t \log (\log (t)))^{\frac{2 \log (t)^{\gamma-\delta}}{\left(1-A^{-1}\right)}} \bar{\Pi}\left(g_{\delta}(t)\right) \log (t)^{\gamma-\delta} .
\end{gathered}
$$

Recall $1+\gamma-\delta=0$. Noting that $t \log (\log (t)) \lesssim t^{2}$ uniformly in $t>t_{0}(y)>0$, it follows that

$$
\begin{aligned}
(*) & \lesssim t \log (t)^{1+\gamma-\delta} t^{\frac{4 \log (t)^{\gamma-\delta}}{1-A^{-1}}} \bar{\Pi}\left(g_{\delta}(t)\right)=t e^{\frac{4\left(1-A^{-1}\right) \log (t)^{1+\gamma-\delta}}{1-A^{-1}}} \bar{\Pi}\left(g_{\delta}(t)\right) \\
& =t e^{\frac{4\left(1-A^{-1}\right)}{1-A^{-1}}} \bar{\Pi}\left(g_{\delta}(t)\right) .
\end{aligned}
$$

Now, since $\delta<\beta$, by (3.1), $\lim _{t \rightarrow \infty} t \bar{\Pi}\left(g_{\delta}(t)\right)=0$, and hence $\lim _{t \rightarrow \infty}(*)=0$, so by Lemma for suitable $u,\left(S_{2}^{*}\right) \leq \Phi_{y}^{h}(t) u(t) \bar{\Pi}(g(t)) /(f(y)-h)$, uniformly in $(h, y, t) \in \mathcal{S}$, as required for (5.12).

\section{Proof of Lemma 4.7}

Proof of Lemma 4.\%: Let $T_{g(h)}$ denote the time when $X$ first passes above $g(h)$, and let $S_{\Delta^{g(h)}}$ be the size of $X$ 's first jump of size larger than $g(h)$. For each $y>K$, with $K>0$ a large, fixed constant,

$$
\begin{aligned}
\mathbb{P}\left(X_{h} \in g(h) d y ; \mathcal{O}_{h}\right) & =\mathbb{P}\left(X_{h} \in g(h) d y ; X_{T_{g(h)}} \leq \frac{g(h) y}{2} ; \mathcal{O}_{h}\right) \\
& +\mathbb{P}\left(X_{h} \in g(h) d y ; X_{T_{g(h)}}>\frac{g(h) y}{2} ; S_{\Delta_{1}^{g(h)}}<\frac{g(h) y}{2} ; \mathcal{O}_{h}\right) \\
& +\mathbb{P}\left(X_{h} \in g(h) d y ; X_{T_{g(h)}}>\frac{g(h) y}{2} ; S_{\Delta_{1}^{g(h)}} \geq \frac{g(h) y}{2} ; \mathcal{O}_{h}\right) \\
& =: \sigma_{h}^{1}(d y)+\sigma_{h}^{2}(d y)+\sigma_{h}^{3}(d y) .
\end{aligned}
$$

We will bound $\mathbb{P}\left(X_{h} \in g(h) d y ; \mathcal{O}_{h}\right)$ by bounding these 3 terms separately. 
Upper Bound for $\sigma_{h}^{1}(d y)$. We shall disintegrate on the values of $T_{g(h)}$ and $X_{T_{g(h)}}$. Observe by (3.4) that $\mathbb{P}\left(\mathcal{O}_{h} ; T_{g(h)} \in d s\right)=\mathbb{P}\left(\mathcal{O}_{s} ; T_{g(h)} \in d s\right)$, so that we can apply (5.3) and the independent increments property, with the notation $\mathbb{P}\left(X_{t} \in d x\right)=$ $f_{t}(x) d x$, to yield

$$
\begin{aligned}
\sigma_{h}^{1}(d y) & =\int_{s=0}^{h} \int_{w=1}^{\frac{y}{2}} \mathbb{P}\left(X_{h} \in g(h) d y ; X_{T_{g(h)}} \in g(h) d w ; T_{g(h)} \in d s ; \mathcal{O}_{h}\right) \\
& =\int_{0}^{h} \int_{1}^{\frac{y}{2}} f_{h-s}(g(h)(y-w)) g(h) d y \mathbb{P}\left(X_{T_{g(h)}} \in g(h) d w ; T_{g(h)} \in d s ; \mathcal{O}_{s}\right) \\
& =\int_{0}^{h} \int_{1}^{\frac{y}{2}} f_{h-s}(g(h)(y-w)) g(h) d y \mathbb{P}\left(X_{T_{g(h)}} \in g(h) d w ; T_{g(h)} \in d s ; \mathcal{O}_{h}\right) .
\end{aligned}
$$

Now, $g(h)(y-w)>g(h) y / 2>g(h)+x_{0} \geq g(h-s)+x_{0}$, for all large enough $h$, with $x_{0}$ as in Assumption 3.3, so (3.2) applies to $f_{h-s}(g(h)(y-w))$. Applying (4.30), since $y-w \geq y / 2$ and $L$ is slowly varying at $\infty$, uniformly in $y>K$ by Bingham et al. (1989, Theorem 1.2.1), as $h \rightarrow \infty$,

$$
\begin{aligned}
\sigma_{h}^{1}(d y) \stackrel{(3.2)}{\lesssim} \int_{0}^{h} \int_{1}^{\frac{y}{2}}(h-s) u(g(h)(y-w)) g(h) d y \mathbb{P}\left(X_{T_{g(h)}} \in g(h) d w ; T_{g(h)} \in d s ; \mathcal{O}_{h}\right) \\
\stackrel{(4.30)}{\lesssim} \int_{0}^{h} \int_{1}^{\frac{y}{2}} \frac{(h-s)}{g(h)^{\alpha}}(y-w)^{-1-\alpha} L(g(h)(y-w)) d y \\
\quad \times \mathbb{P}\left(X_{T_{g(h)}} \in g(h) d w ; T_{g(h)} \in d s ; \mathcal{O}_{h}\right) \\
\lesssim \int_{0}^{h} \int_{1}^{\frac{y}{2}} \frac{h}{g(h)^{\alpha}} \frac{L(g(h))}{L(g(h))} y^{-1-\alpha} L\left(\frac{g(h) y}{2}\right) d y \\
\quad \times \mathbb{P}\left(X_{T_{g(h)}} \in g(h) d w ; T_{g(h)} \in d s ; \mathcal{O}_{h}\right) \\
\lesssim y^{-1-\alpha} \frac{L(g(h) y)}{L(g(h))} d y \frac{h L(g(h))}{g(h)^{\alpha}} \int_{0}^{h} \int_{1}^{\frac{y}{2}} \mathbb{P}\left(X_{T_{g(h)}} \in g(h) d w ; T_{g(h)} \in d s ; \mathcal{O}_{h}\right) \\
\leq y^{-1-\alpha} \frac{L(g(h) y)}{L(g(h))} d y h \bar{\Pi}(g(h)) \mathbb{P}\left(\mathcal{O}_{h}\right) \\
\stackrel{(3.1)}{=} o(1) \times y^{-1-\alpha} \frac{L(g(h) y)}{L(g(h))} \mathbb{P}\left(\mathcal{O}_{h}\right) d y
\end{aligned}
$$

where, recalling $g(h)^{-\alpha} L(g(h))=\bar{\Pi}(g(h))$, the last step follows by (3.1).

Simplifying the Expressions for $\sigma_{h}^{2}(d y)$ and $\sigma_{h}^{3}(d y)$. Recall (3.7), and $\sigma_{h}^{2}(d y)+$ $\sigma_{h}^{3}(d y)=\mathbb{P}\left(X_{h} \in g(h) d y ; X_{T_{g(h)}}>g(h) y / 2 ; \mathcal{O}_{h}\right)$. Choosing $K>4$, we have $g(h) y / 2>2 g(h)$ for $y>K$. As $T_{g(h)}$ is the first passage time above $g(h)$, if $X_{T_{g(h)}}>2 g(h)$, then $X$ crosses $g(h)$ by a jump larger than $g(h)$, so since $T_{g(h)} \leq \Delta_{1}^{g(h)}, T_{g(h)}=\Delta_{1}^{g(h)}$.

Then since $X_{t}<g(h)$ for all $t<T_{g(h)}$, it follows that $X_{T_{g(h)}-}=X_{\Delta_{1}^{g(h)}-}<g(h)$, as $X$ has càdlàg sample paths, almost surely. Moreover, if $\mathcal{O}_{h}$ holds, then $X$ crosses 
$g(h)$ by time $h$, so $\Delta_{1}^{g(h)}=T_{g(h)} \leq h$. Thus:

$$
\begin{aligned}
& \left\{X_{T_{g(h)}}>\frac{g(h) y}{2} ; S_{\Delta_{1}^{g(h)}}<\frac{g(h) y}{2} ; \mathcal{O}_{h}\right\} \\
& \subseteq\left\{\Delta_{1}^{g(h)} \leq h ; X_{\Delta_{1}^{g(h)}-}<g(h) ; S_{\Delta_{1}^{g(h)}}<\frac{g(h) y}{2} ; \mathcal{O}_{h}\right\},
\end{aligned}
$$

and therefore we can bound $\sigma_{h}^{2}(d y)$ by

$$
\begin{aligned}
\sigma_{h}^{2}(d y) & =\mathbb{P}\left(X_{h} \in g(h) d y ; X_{T_{g(h)}}>\frac{g(h) y}{2} ; S_{\Delta_{1}^{g(h)}}<\frac{g(h) y}{2} ; \mathcal{O}_{h}\right) \\
& \leq \mathbb{P}\left(X_{h} \in g(h) d y ; \Delta_{1}^{g(h)} \leq h ; X_{\Delta_{1}^{g(h)}-}<g(h) ; S_{\Delta_{1}^{g(h)}}<\frac{g(h) y}{2} ; \mathcal{O}_{h}\right) .
\end{aligned}
$$

For $\sigma_{h}^{3}(d y)$, the converse analogous inclusion to (6.4) holds too, that is, if $\mathcal{O}_{h}$, $\Delta_{1}^{g(h)} \leq h, X_{\Delta_{1}^{g(h)}-}<g(h)$, and $S_{\Delta_{1}^{g(h)}} \geq g(h) y / 2$ hold, then we have

$$
X_{T_{g(h)}}=X_{\Delta_{1}^{g(h)}} \geq X_{\Delta_{1}^{g(h)}}-X_{\Delta_{1}^{g(h)}-}=S_{\Delta_{1}^{g(h)}}>g(h) y / 2,
$$

and therefore $\sigma_{h}^{3}(d y)$ satisfies

$$
\begin{aligned}
\sigma_{h}^{3}(d y) & =\mathbb{P}\left(X_{h} \in g(h) d y ; X_{T_{g(h)}}>\frac{g(h) y}{2} ; S_{\Delta_{1}^{g(h)}} \geq \frac{g(h) y}{2} ; \mathcal{O}_{h}\right) \\
& =\mathbb{P}\left(X_{h} \in g(h) d y ; \Delta_{1}^{g(h)} \leq h ; X_{\Delta_{1}^{g(h)} \_}<g(h) ; S_{\Delta_{1}^{g(h)}} \geq \frac{g(h) y}{2} ; \mathcal{O}_{h}\right) .
\end{aligned}
$$

Upper Bound for $\sigma_{h}^{2}(d y)$. By (6.5) and (5.5), disintegrating on the values of $\Delta_{1}^{g(h)}$, $X_{\Delta_{1}^{g(h)}-}$, and $S_{\Delta_{1}^{g(h)}}$, by independence of increments and the Markov property, with $\mathbb{P}\left(X_{t} \in d x\right)=f_{t}(x) d x$,

$$
\begin{aligned}
& \sigma_{h}^{2}(d y) \stackrel{(6.5)}{\leq} \mathbb{P}\left(X_{h} \in g(h) d y ; \Delta_{1}^{g(h)} \leq h ; X_{\Delta_{1}^{g(h)}-}<g(h) ; S_{\Delta_{1}^{g(h)}}<\frac{g(h) y}{2} ; \mathcal{O}_{h}\right) \\
& \stackrel{(5.5)}{=} \int_{s=0}^{h} \int_{w=0}^{1} \int_{v=0}^{\frac{y}{2}} \mathbb{P}\left(X_{h} \in g(h) d y ; \Delta_{1}^{g(h)} \in d s ; X_{\Delta_{1}^{g(h)}-} \in g(h) d w ;\right. \\
& \left.S_{\Delta_{1}^{g(h)}} \in g(h) d v ; \mathcal{O}_{s}\right) \\
& =\int_{0}^{h} \int_{0}^{1} \int_{0}^{\frac{y}{2}} f_{h-s}(g(h)(y-w-v)) g(h) d y \\
& \times \mathbb{P}\left(\Delta_{1}^{g(h)} \in d s ; X_{\Delta_{1}^{g(h)}-} \in g(h) d w ; S_{\Delta_{1}^{g(h)}} \in g(h) d v ; \mathcal{O}_{s}\right) \\
& \stackrel{(5.5)}{=} \int_{0}^{h} \int_{0}^{1} \int_{0}^{\frac{y}{2}} f_{h-s}(g(h)(y-w-v)) g(h) d y \\
& \times \mathbb{P}\left(\Delta_{1}^{g(h)} \in d s ; X_{\Delta_{1}^{g(h)}-} \in g(h) d w ; S_{\Delta_{1}^{g(h)}} \in g(h) d v ; \mathcal{O}_{h}\right) .
\end{aligned}
$$

Note $y-w-v>y / 3>K / 3$ for $w \leq 1, v \leq y / 2$. So as $h \rightarrow \infty, g(h)(y-w-v) \geq$ $g(h-s)+x_{0}$, so we can apply (3.2) and (4.30). Now, $g(h)^{-\alpha} L(g(h))=\bar{\Pi}(g(h))$ for $L$ slowly varying at $\infty$, so by (3.1), uniformly in $y>K$ by the uniform convergence theorem (Bingham et al., 1989, Theorem 1.2.1), as $h \rightarrow \infty$,

$$
\sigma_{h}^{2}(d y) \stackrel{(3.2)}{\lesssim} \int_{0}^{h} \int_{0}^{1} \int_{0}^{\frac{y}{2}}(h-s) u(g(h)(y-w-v)) g(h) d y
$$




$$
\begin{aligned}
& \times \mathbb{P}\left(\Delta_{1}^{g(h)} \in d s ; X_{\Delta_{1}^{g(h)}-} \in g(h) d w ; S_{\Delta_{1}^{g(h)}} \in g(h) d v ; \mathcal{O}_{h}\right) \\
& \stackrel{(4.30)}{\lesssim} \int_{0}^{h} \int_{0}^{1} \int_{0}^{\frac{y}{2}} \frac{(h-s) L(g(h)(y-w-v))}{g(h)^{\alpha}(y-w-v)^{1+\alpha}} d y \times \mathbb{P}\left(\Delta_{1}^{g(h)} \in d s ; X_{\Delta_{1}^{g(h)}-} \in g(h) d w ; S_{\Delta_{1}^{g(h)}} \in g(h) d v ; \mathcal{O}_{h}\right) \\
& \lesssim \int_{0}^{h} \int_{0}^{1} \int_{0}^{\frac{y}{2}} \frac{(h-s)}{g(h)^{\alpha} y^{1+\alpha}} L(g(h) y) d y \\
& \quad \times \mathbb{P}\left(\Delta_{1}^{g(h)} \in d s ; X_{\Delta_{1}^{g(h)}-} \in g(h) d w ; S_{\Delta_{1}^{g(h)}} \in g(h) d v ; \mathcal{O}_{h}\right) \\
& \leq \frac{h L(g(h))}{g(h)^{\alpha}} \int_{0}^{h} \int_{0}^{1} \int_{0}^{\frac{y}{2}} y^{-1-\alpha} \frac{L(g(h) y)}{L(g(h)} d y \\
& \quad \times \mathbb{P}\left(\Delta_{1}^{g(h)} \in d s ; X_{\Delta_{1}^{g(h)}-} \in g(h) d w ; S_{\Delta_{1}^{g(h)}} \in g(h) d v ; \mathcal{O}_{h}\right) \\
& \stackrel{(3.1)}{=} o(1) \times \int_{0}^{h} \int_{0}^{1} \int_{0}^{\frac{y}{2}} y^{-1-\alpha} \frac{L(g(h) y)}{L(g(h)} d y \\
& \quad \times \mathbb{P}\left(\Delta_{1}^{g(h)} \in d s ; X_{\Delta_{1}^{g(h)}-} \in g(h) d w ; S_{\Delta_{1}^{g(h)}} \in g(h) d v ; \mathcal{O}_{h}\right) \\
& \leq o(1) \times y^{-1-\alpha} \frac{L(g(h) y)}{L(g(h))} \mathbb{P}\left(\mathcal{O}_{h}\right) d y .
\end{aligned}
$$

Upper Bound for $\sigma_{h}^{3}(d y)$. Disintegrating on the values of $\Delta_{1}^{g(h)}, X_{\Delta_{1}^{g(h)}-}$ and $S_{\Delta_{1}^{g(h)}}$, then applying (5.5), independence of increments, the Markov property, and Lemma 4.9 , it follows that uniformly among $y>K$ as $h \rightarrow \infty$, with $\mathbb{P}\left(X_{t} \in d x\right)=f_{t}(x) d x$,

$$
\begin{aligned}
& \sigma_{h}^{3}(d y)= \int_{s=0}^{h} \int_{w=0}^{1} \int_{v=\frac{y}{2}}^{y-w} \mathbb{P}\left(X_{h} \in g(h) d y ; \Delta_{1}^{g(h)} \in d s ;\right. \\
&\left.X_{\Delta_{1}^{g(h)}-} \in g(h) d w ; S_{\Delta_{1}^{g(h)}} \in g(h) d v ; \mathcal{O}_{h}\right) \\
& \stackrel{(5.5)}{=} \int_{0}^{h} \int_{0}^{1} \int_{\frac{y}{2}}^{y-w} f_{h-s}(g(h)(y-w-v)) g(h) d y \mathbb{P}\left(S_{\Delta_{1}^{g(h)}} \in g(h) d v\right) \\
& \times \mathbb{P}\left(\Delta_{1}^{g(h)} \in d s ; X_{\Delta_{1}^{g(h)}-} \in g(h) d w ; \mathcal{O}_{s}\right) \\
& \stackrel{4.9}{\lesssim} \int_{0}^{h} \int_{0}^{1} \int_{\frac{y}{2}-w}^{y-w} f_{h-s}(g(h)(y-w-v)) g(h) d y v^{-1-\alpha} \frac{L(g(h) v)}{L(g(h))} d v \\
& \stackrel{(5.5)}{=} \int_{0}^{h} \int_{0}^{1} \int_{\frac{y}{2}-w}^{y-w} f_{h-s}(g(h)(y-w-v)) g(h) d y v^{-1-\alpha} \frac{L(g(h) v)}{L(g(h))} d v \\
& \times \mathbb{P}\left(\Delta_{1}^{g(h)} \in d s ; X_{\Delta_{1}^{g(h)}-} \in g(h) d w ; \mathcal{O}_{h}\right) .
\end{aligned}
$$

Now, as $y / 3 \leq y / 2-1 \leq y / 2-w \leq v \leq y$, applying the uniform convergence theorem Bingham et al. (1989, Theorem 1.2.1) to $L(g(h) v) / L(g(h) y)$, uniformly in $y>K$ as $h \rightarrow \infty$,

$$
\sigma_{h}^{3}(d y) \lesssim y^{-1-\alpha} \frac{L(g(h) y)}{L(g(h))} d y \int_{0}^{h} \int_{0}^{1} \int_{\frac{y}{2}-w}^{y-w} f_{h-s}(g(h)(y-w-v)) g(h) d v
$$




$$
\times \mathbb{P}\left(\Delta_{1}^{g(h)} \in d s ; X_{\Delta_{1}^{g(h)}-} \in g(h) d w ; \mathcal{O}_{h}\right) .
$$

Changing variables to $u=g(h)(y-w-v)$, uniformly in $y>K$, as $h \rightarrow \infty$,

$$
\begin{aligned}
\sigma_{h}^{3}(d y) \lesssim & y^{-1-\alpha} \frac{L(g(h) y)}{L(g(h))} d y \int_{0}^{h} \int_{0}^{1} \int_{0}^{\frac{g(h) y}{2}} f_{h-s}(u) d u \\
& \times \mathbb{P}\left(\Delta_{1}^{g(h)} \in d s ; X_{\Delta_{1}^{g(h)}-} \in g(h) d w ; \mathcal{O}_{h}\right) \\
= & y^{-1-\alpha} \frac{L(g(h) y)}{L(g(h))} d y \int_{0}^{h} \int_{0}^{1} \mathbb{P}\left(X_{h-s} \leq \frac{g(h) y}{2}\right) \\
& \times \mathbb{P}\left(\Delta_{1}^{g(h)} \in d s ; X_{\Delta_{1}^{g(h)}} \in g(h) d w ; \mathcal{O}_{h}\right) \\
\leq & y^{-1-\alpha} \frac{L(g(h) y)}{L(g(h))} d y \int_{0}^{h} \int_{0}^{1} \mathbb{P}\left(\Delta_{1}^{g(h)} \in d s ; X_{\Delta_{1}^{g(h)}-} \in g(h) d w ; \mathcal{O}_{h}\right) \\
= & y^{-1-\alpha} \frac{L(g(h) y)}{L(g(h))} d y \mathbb{P}\left(\Delta_{1}^{g(h)} \leq h ; X_{\Delta_{1}^{g(h)}-}<g(h) ; \mathcal{O}_{h}\right) \\
\leq & y^{-1-\alpha} \frac{L(g(h) y)}{L(g(h))} \mathbb{P}\left(\mathcal{O}_{h}\right) d y .
\end{aligned}
$$

Conclusion of Upper Bound. By (6.1), (6.3), (6.7), and (6.10), we conclude, as required for the upper bound in (4.29), that uniformly in $y>K$, as $h \rightarrow \infty$,

$$
\mathbb{P}\left(X_{h} \in g(h) d y ; \mathcal{O}_{h}\right) \lesssim y^{-1-\alpha} \frac{L(g(h) y)}{L(g(h))} \mathbb{P}\left(\mathcal{O}_{h}\right) d y
$$

Now we will prove the lower bound on $\mathbb{P}\left(X_{h} \in g(h) d y ; \mathcal{O}_{h}\right)$.

Proof of Lower Bound. Now, fixing $y_{0}>0$, for all $y>K$, as $h \rightarrow \infty$,

$$
\begin{aligned}
\mathbb{P}\left(X_{h} \in g(h) d y ; \mathcal{O}_{h}\right) \geq \mathbb{P}\left(X_{h} \in g(h) d y ; \Delta_{1}^{g(h)}\right. & \leq h-1 ; X_{\Delta_{1}^{g(h)}-}<g(h) ; \\
\frac{g(h) y}{2} & \left.\leq S_{\Delta_{1}^{g(h)}} \leq g(h) y-y_{0} ; \mathcal{O}_{h}\right) .
\end{aligned}
$$

Disintegrating on the values of $\Delta_{1}^{g(h)}, X_{\Delta_{1}^{g(h)}-}$, and $S_{\Delta_{1}^{g(h)}}$, applying the Markov property, noting that by (5.5), $\left\{\Delta_{1}^{g(h)} \in d s ; \mathcal{O}_{h}\right\}=\left\{\Delta_{1}^{g(h)} \in d s ; \mathcal{O}_{\Delta_{1}^{g(h)}}\right\}$ for each $s \leq h$, with $\mathbb{P}\left(X_{t} \in d x\right)=f_{t}(x) d x$,

$$
\begin{gathered}
(6.12) \stackrel{(5.5)}{=} \int_{s=0}^{h-1} \int_{w=0}^{1} \int_{v=\frac{y}{2}}^{y-\frac{y_{0}}{g(h)}-w} \mathbb{P}\left(X_{h} \in g(h) d y ; \Delta_{1}^{g(h)} \in d s ; X_{\Delta_{1}^{g(h)}-} \in g(h) d w ;\right. \\
\left.S_{\Delta_{1}^{g(h)}} \in g(h) d v ; \mathcal{O}_{\Delta_{1}^{g(h)}}\right) \\
\stackrel{(5.5)}{=} \int_{0}^{h-1} \int_{0}^{1} \int_{v=\frac{y}{2}}^{y-\frac{y_{0}}{g(h)}-w} f_{h-s}(g(h)(y-w-v)) g(h) d y \mathbb{P}\left(S_{\Delta_{1}^{g(h)}} \in g(h) d v\right) \\
\times \mathbb{P}\left(\Delta_{1}^{g(h)} \in d s ; X_{\Delta_{1}^{g(h)}-} \in g(h) d w ; \mathcal{O}_{h}\right) .
\end{gathered}
$$


Applying Lemma 4.9, noting $h-s \geq 1, y / 2<2 y / 3-w, v \asymp y$, and $L(g(h) v) \asymp$ $L(g(h) y)$ uniformly in $y>K$ as $h \rightarrow \infty$ by the uniform convergence theorem Bingham et al. (1989, Thm 1.2.1), it follows that uniformly in $y>K$ as $h \rightarrow \infty$,

$$
\begin{gathered}
(6.12) \gtrsim \int_{0}^{4.9} \int_{0}^{h-1} \int_{v=\frac{y}{2}}^{y-\frac{y_{0}}{g(h)}-w} f_{h-s}(g(h)(y-w-v)) g(h) d y v^{-1-\alpha} \frac{L(g(h) v)}{L(g(h))} d v \\
\times \mathbb{P}\left(\Delta_{1}^{g(h)} \in d s ; X_{\Delta_{1}^{g(h)}-} \in g(h) d w ; \mathcal{O}_{h}\right) \\
\gtrsim \int_{0}^{h-1} \int_{0}^{1} \int_{v=\frac{2 y}{3}-w}^{y-\frac{y_{0}}{g(h)}-w} f_{h-s}(g(h)(y-w-v)) g(h) d y y^{-1-\alpha} \frac{L(g(h) y)}{L(g(h))} d v \\
\times \mathbb{P}\left(\Delta_{1}^{g(h)} \in d s ; X_{\Delta_{1}^{g(h)}-} \in g(h) d w ; \mathcal{O}_{h}\right) .
\end{gathered}
$$

Changing variables to $u=g(h)(y-w-v)$, noting that $y / 3>1$ for all $y>K$ and that $h-s \geq 1$,

$$
\begin{aligned}
&(6.12) \gtrsim y^{-1-\alpha} \frac{L(g(h) y)}{L(g(h))} d y \int_{0}^{h-1} \int_{0}^{1} \int_{u=y_{0}}^{\frac{g(h) y}{3}} f_{h-s}(u) d u \\
& \times \mathbb{P}\left(\Delta_{1}^{g(h)} \in d s ; X_{\Delta_{1}^{g(h)}-} \in g(h) d w ; \mathcal{O}_{h}\right) \\
&=y^{-1-\alpha} \frac{L(g(h) y)}{L(g(h))} d y \int_{0}^{h-1} \int_{0}^{1}\left[\mathbb{P}\left(X_{h-s} \leq \frac{g(h) y}{3}\right)-\mathbb{P}\left(X_{h-s} \leq y_{0}\right)\right] \\
& \times \mathbb{P}\left(\Delta_{1}^{g(h)} \in d s ; X_{\Delta_{1}^{g(h)}-} \in g(h) d w ; \mathcal{O}_{h}\right) \\
& \geq y^{-1-\alpha} \frac{L(g(h) y)}{L(g(h))} d y \int_{0}^{h-1} \int_{0}^{1}\left[\mathbb{P}\left(X_{h} \leq g(h)\right)-\mathbb{P}\left(X_{1} \leq y_{0}\right)\right] \\
& \times \mathbb{P}\left(\Delta_{1}^{g(h)} \in d s ; X_{\Delta_{1}^{g(h)}-} \in g(h) d w ; \mathcal{O}_{h}\right) .
\end{aligned}
$$

Now, with $X^{(0, g(h))}$ again denoting the process with no jumps bigger than $g(h)$,

$$
\begin{aligned}
\mathbb{P}\left(X_{h} \leq g(h)\right) & =\mathbb{P}\left(X_{h}^{(0, g(h))} \leq g(h)\right) \mathbb{P}\left(\Delta_{1}^{g(h)}>h\right) \\
& =\mathbb{P}\left(X_{h}^{(0, g(h))} \leq g(h)\right) e^{-h \bar{\Pi}(g(h))},
\end{aligned}
$$

and since $\lim _{h \rightarrow \infty} h \bar{\Pi}(g(h))=0$ by (3.1), by Markov's inequality, as $h \rightarrow \infty$,

$$
\mathbb{P}\left(X_{h} \leq g(h)\right) \stackrel{(3.1)}{\sim} \mathbb{P}\left(X_{h}^{(0, g(h))} \leq g(h)\right) \geq 1-\frac{\mathbb{E}\left[X_{h}^{(0, g(h))}\right]}{g(h)} \geq 1-\frac{h \int_{0}^{g(h)} \bar{\Pi}(x) d x}{g(h)} .
$$

By (3.1) and Karamata's theorem (Bingham et al., 1989, Prop 1.5.8), as $h \rightarrow \infty$,

$$
\mathbb{P}\left(X_{h} \leq g(h)\right) \gtrsim 1-\frac{h g(h) \bar{\Pi}(g(h))}{g(h)}=1-h \bar{\Pi}(g(h)) \stackrel{(3.1)}{\sim} 1 .
$$

Then as $\mathbb{P}\left(X_{1} \leq y_{0}\right)=$ constant $<1$, taking $y_{0}$ large enough that $\mathbb{P}\left(X_{h} \leq g(h)\right)$ $\mathbb{P}\left(X_{1} \leq y_{0}\right) \gtrsim 1$ uniformly, we get that uniformly in $y>K$ as $h \rightarrow \infty$,

$$
\begin{aligned}
(6.12) & \gtrsim \frac{L(g(h) y)}{L(g(h))} y^{-1-\alpha} d y \int_{s=0}^{h-1} \int_{w=0}^{1} \mathbb{P}\left(\Delta_{1}^{g(h)} \in d s ; X_{\Delta_{1}^{g(h)}-} \in g(h) d w ; \mathcal{O}_{h}\right) \\
& =\frac{L(g(h) y)}{L(g(h))} y^{-1-\alpha} d y \mathbb{P}\left(\Delta_{1}^{g(h)} \leq h-1 ; X_{\Delta_{1}^{g(h)}-}<g(h) ; \mathcal{O}_{h}\right) .
\end{aligned}
$$


Proof by Contradiction Step. Now we assume for a contradiction that

$$
\liminf _{h \rightarrow \infty} \frac{\mathbb{P}\left(\Delta_{1}^{g(h)} \leq h-1 ; X_{\Delta_{1}^{g(h)}-}<g(h) ; \mathcal{O}_{h}\right)}{\mathbb{P}\left(\mathcal{O}_{h}\right)}=0 .
$$

As $\Delta_{1}^{g(h)}$ is exponentially distributed with rate $\bar{\Pi}(g(h))$, by Corollary 4.4 , as $h \rightarrow \infty$,

$$
\begin{aligned}
& \mathbb{P}\left(\Delta_{1}^{g(h)} \in[h-1, h] ; X_{\Delta_{1}^{g(h)}-}<g(h) ; \mathcal{O}_{h}\right) \leq \mathbb{P}\left(\Delta_{1}^{g(h)} \in[h-1, h]\right) \\
& \leq \mathbb{P}\left(\Delta_{1}^{g(h)} \leq 1\right)=1-e^{-\bar{\Pi}(g(h))} \leq \bar{\Pi}(g(h)) \stackrel{4.4}{\sim} \frac{\mathbb{P}\left(\mathcal{O}_{h}\right)}{\Phi(h)} \stackrel{(4.5)}{=} o(1) \times \mathbb{P}\left(\mathcal{O}_{h}\right),
\end{aligned}
$$

since $\lim _{h \rightarrow \infty} \Phi(h)=\infty$ by (4.5), so it follows that (6.14) holds if and only if

$$
\liminf _{h \rightarrow \infty} \frac{\mathbb{P}\left(\Delta_{1}^{g(h)} \leq h ; X_{\Delta_{1}^{g(h)}-}<g(h) ; \mathcal{O}_{h}\right)}{\mathbb{P}\left(\mathcal{O}_{h}\right)}=0 .
$$

By (6.1), (6.3), (6.7), and (6.9), we get that (6.15) implies, along a subsequence of $h$, as $h \rightarrow \infty$,

$$
\begin{aligned}
\mathbb{P}\left(X_{h} \geq K g(h) ; \mathcal{O}_{h}\right) & =\int_{K}^{\infty} \mathbb{P}\left(X_{h} \in g(h) d y ; \mathcal{O}_{h}\right) \\
& =o(1) \times \mathbb{P}\left(\mathcal{O}_{h}\right) \int_{K}^{\infty} y^{-1-\alpha} \frac{L(g(h) y)}{L(g(h))} d y .
\end{aligned}
$$

Changing variables from $y$ to $u=g(h) y$,

$$
\mathbb{P}\left(X_{h} \geq K g(h) ; \mathcal{O}_{h}\right)=o(1) \times \frac{g(h)^{\alpha} \mathbb{P}\left(\mathcal{O}_{h}\right)}{L(g(h))} \int_{K g(h)}^{\infty} u^{-1-\alpha} L(u) d u .
$$

As $L$ is slowly varying, applying the result Bingham et al. (1989, Prop 1.5.10) to $\int_{K g(h)}^{\infty} u^{-1-\alpha} L(u) d u$, as $h \rightarrow \infty$,

$$
\mathbb{P}\left(X_{h} \geq K g(h) ; \mathcal{O}_{h}\right) \lesssim o(1) \times \frac{g(h)^{\alpha} \mathbb{P}\left(\mathcal{O}_{h}\right)}{L(g(h))}(K g(h))^{-\alpha} L(K g(h))=o(1) \times \mathbb{P}\left(\mathcal{O}_{h}\right) .
$$

But considering the subevent $\left\{\Delta_{1}^{g(h)}=\Delta_{1}^{K g(h)} \leq h ; \mathcal{O}_{h}\right\} \subseteq\left\{X_{h} \geq K g(h) ; \mathcal{O}_{h}\right\}$, disintegrating on the value of $\Delta_{1}^{g(h)}$, and applying the Markov property,

$$
\begin{aligned}
\mathbb{P}\left(X_{h} \geq K g(h) ; \mathcal{O}_{h}\right) & \geq \mathbb{P}\left(\Delta_{1}^{g(h)}=\Delta_{1}^{K g(h)} \leq h ; \mathcal{O}_{h}\right) \\
& =\int_{0}^{h} \mathbb{P}\left(\Delta_{1}^{g(h)}=\Delta_{1}^{K g(h)} \in d s ; \mathcal{O}_{h}\right) \\
& =\int_{0}^{h} \mathbb{P}\left(\Delta_{1}^{g(h)} \in d s ; S_{\Delta_{1}^{g(h)}} \geq K g(h) ; \mathcal{O}_{s, X^{(0, g(h))}}\right) \\
& =\int_{0}^{h} \mathbb{P}\left(\mathcal{O}_{s, X}(0, g(h)) ; \Delta_{1}^{g(h)} \in d s\right) \mathbb{P}\left(S_{\Delta_{1}^{g(h)}} \geq K g(h)\right) \\
& =\frac{\bar{\Pi}(K g(h))}{\bar{\Pi}(g(h))} \int_{0}^{h} \mathbb{P}\left(\mathcal{O}_{s, X^{(0, g(h))}} ; \Delta_{1}^{g(h)} \in d s\right)
\end{aligned}
$$




$$
\begin{aligned}
& =\frac{\bar{\Pi}(K g(h))}{\bar{\Pi}(g(h))} \int_{0}^{h} \mathbb{P}\left(\mathcal{O}_{h} ; \Delta_{1}^{g(h)} \in d s\right) \\
& =\frac{\bar{\Pi}(K g(h))}{\bar{\Pi}(g(h))} \mathbb{P}\left(\mathcal{O}_{h} ; \Delta_{1}^{g(h)} \leq h\right) .
\end{aligned}
$$

By (5.8) with $g_{y}^{h}(t)=g(t)$, and by Corollary 4.4, $\mathbb{P}\left(\mathcal{O}_{h} ; \Delta_{1}^{g(h)} \leq h\right) \sim \mathbb{P}\left(\mathcal{O}_{h}\right)$ as $h \rightarrow \infty$, so as $h \rightarrow \infty$,

$$
\mathbb{P}\left(X_{h} \geq K g(h) ; \mathcal{O}_{h}\right) \geq \frac{\bar{\Pi}(K g(h))}{\bar{\Pi}(g(h))} \mathbb{P}\left(\mathcal{O}_{h}\right) \sim K^{-\alpha} \mathbb{P}\left(\mathcal{O}_{h}\right),
$$

because $\bar{\Pi}$ is regularly varying at $\infty$, so (6.16) contradicts (6.17), and therefore $\liminf \operatorname{in}_{h \rightarrow \infty} \mathbb{P}\left(\Delta_{1}^{g(h)} \leq h ; X_{\Delta_{1}^{g(h)}{ }_{-}}<g(h) ; \mathcal{O}_{h}\right) / \mathbb{P}\left(\mathcal{O}_{h}\right)>0$. By (6.13), uniformly in $y>K$ as $h \rightarrow \infty$,

$$
\mathbb{P}\left(X_{h} \in g(h) d y ; \mathcal{O}_{h}\right) \gtrsim y^{-1-\alpha} \frac{L(g(h) y)}{L(g(h))} \mathbb{P}\left(\mathcal{O}_{h}\right) d y,
$$

as required for the lower bound in (4.29), so the proof of Lemma 4.7 is complete.

\section{Proofs of Auxiliary Lemmas}

Proof of Lemma 4.3: Firstly, we will show that $\rho(t)=o(\bar{\Pi}(g(t)))$ as $t \rightarrow \infty$. By (4.2) with $y=h=0$, it is immediate that $\lim _{s \rightarrow \infty} \rho(s) / \bar{\Pi}(g(s)) \leq 0$. Now, by (3.13) and (5.8),

$$
\begin{aligned}
-\rho(s) & =\frac{1}{\Phi(s)}\left[\bar{\Pi}(g(s))^{2} \int_{0}^{s} \int_{0}^{v} \mathbb{P}\left(\mathcal{O}_{w}\right) e^{-\bar{\Pi}(g(s)) w} d w d v-\mathbb{P}\left(\mathcal{O}_{s} ; \Delta_{1}^{g(s)}>s\right)\right] \\
& \leq \frac{1}{\Phi(s)} \bar{\Pi}(g(s))^{2} \int_{0}^{s} \int_{0}^{v} \mathbb{P}\left(\mathcal{O}_{w}\right) e^{-\bar{\Pi}(g(s)) w} d w d v \\
& \leq \frac{1}{\Phi(s)} s \bar{\Pi}(g(s))^{2} \int_{0}^{s} \mathbb{P}\left(\mathcal{O}_{w}\right) d w=s \bar{\Pi}(g(s))^{2} .
\end{aligned}
$$

Now, applying (3.1) and (3.3) in cases (i) and (ii) respectively, it follows that $\lim _{s \rightarrow \infty}-\rho(s) / \bar{\Pi}(g(t)) \leq 0$, and hence $\rho(s)=o(\bar{\Pi}(g(s)))$ as $s \rightarrow \infty$.

Next we will show that $-\int_{1}^{\infty} \rho(s) d s<\infty$. Indeed, this follows immediately in case (ii) by (7.1) and (3.3). In case (i), for arbitrarily small $\tau>0$, applying Potter's theorem (Bingham et al., 1989, Theorem 1.5.6) to $\bar{\Pi}(g(s)) / \bar{\Pi}\left(g(s) / \log (s)^{\beta}\right)$, it follows by (7.1) and (3.1) that

$$
\begin{aligned}
-\int_{1}^{\infty} \rho(s) d s & \lesssim \int_{1}^{\infty} s^{2} \bar{\Pi}\left(\frac{g(s)}{\log (s)^{\beta}}\right)^{2} s^{-1} \log (s)^{-2 \alpha \beta+2 \tau} d s \\
& \stackrel{(3.1)}{\lesssim} \int_{1}^{\infty} s^{-1} \log (s)^{-2 \alpha \beta+2 \tau} d s<\infty,
\end{aligned}
$$

where we simply take $\tau$ small enough that $-2 \alpha \beta+2 \tau<-1$, which is possible since $\alpha \beta>1 / 2$. 
Proof of Lemma 4.6: Recall that $\bar{\Pi}(x)=x^{-\alpha} L(x)$ for $L$ slowly varying at $\infty$, so as $\bar{\Pi}$ is non-increasing, for large $N>0$, using that $t_{0}(y) \geq f(A y)$,

$$
\begin{aligned}
& \int_{f(A y)}^{t}(\bar{\Pi}(g(s+h)-y)-\bar{\Pi}(g(s))) d s \leq \int_{f(A y)}^{t}(\bar{\Pi}(g(s)-y)-\bar{\Pi}(g(s))) d s \\
\leq & \int_{f(A y)}^{\infty}(\bar{\Pi}(g(s)-y)-\bar{\Pi}(g(s))) d s=\int_{f(A y)}^{\infty}\left(\frac{L(g(s)-y)}{(g(s)-y)^{\alpha}}-\frac{L(g(s))}{g(s)^{\alpha}}\right) d s \\
= & \int_{f(A y)}^{\infty} \frac{L(g(s)-y)}{(g(s)-y)^{\alpha}}\left(1-\left(\frac{g(s)-y}{g(s)}\right)^{\alpha} \frac{L(g(s))}{L(g(s)-y)}\right) d s \\
= & \int_{f(A y)}^{\infty} \frac{L(g(s)-y)}{(g(s)-y)^{\alpha}}\left(1-\left(\frac{g(s)-y}{g(s)}\right)^{\alpha+N} \frac{g(s)^{N} L(g(s))}{(g(s)-y)^{N} L(g(s)-y)}\right) d s .
\end{aligned}
$$

Now, $A>B-1$, and $x^{N} L(x)$ is non-decreasing in $x$ for $x>B$ in case (i), so

$$
(7.2) \leq \int_{f(A y)}^{\infty} \frac{L(g(s)-y)}{(g(s)-y)^{\alpha}}\left(1-\left(\frac{g(s)-y}{g(s)}\right)^{\alpha+N}\right) d s .
$$

One can verify $1-(1-y / g(s))^{\alpha+N} \lesssim y / g(s)$, uniformly in $y>0, s>f(A y)$, so

$$
(7.2) \lesssim \int_{f(A y)}^{\infty} \frac{L(g(s)-y)}{(g(s)-y)^{\alpha}} \frac{y}{g(s)} d s .
$$

As $g(s)-y \geq\left(1-A^{-1}\right) g(s)$ for $s>f(A y)$, and $\bar{\Pi}(x)=x^{-\alpha} L(x)$ is non-increasing,

$$
(7.2) \lesssim y \int_{f(A y)}^{\infty} \frac{L\left(\left(1-A^{-1}\right) g(s)\right)}{g(s)^{1+\alpha}} d s .
$$

Applying the uniform convergence theorem (Bingham et al., 1989, Theorem 1.2.1) to the slowly varying function $L$, substituting $u=g(s)$, as $u f^{\prime}(u) \bar{\Pi}(u)$ is decreasing, we conclude that uniformly in $y>0$ (and so also uniformly in $h>0, y>g(h)$ ),

$$
\begin{aligned}
(7.2) & \lesssim y \int_{f(A y)}^{\infty} \frac{L(g(s))}{g(s)^{1+\alpha}} d s=y \int_{A y}^{\infty} \frac{L(u)}{u^{1+\alpha}} f^{\prime}(u) d u=y \int_{A y}^{\infty} u^{-2} u f^{\prime}(u) \bar{\Pi}(u) d u \\
& \leq A y^{2} f^{\prime}(A y) \bar{\Pi}(A y) \int_{A y}^{\infty} u^{-2} d u=\frac{1}{2} y f^{\prime}(A y) \bar{\Pi}(A y) \lesssim y f^{\prime}(y) \bar{\Pi}(y) .
\end{aligned}
$$

Proof of Lemma 4.8: First recall that by Theorem 3.14,

$$
q_{h}(y)=\frac{\Phi_{y}^{h}\left(t_{0}(y)\right)}{\Phi(1)} \lim _{t \rightarrow \infty} \exp \left(\int_{t_{0}(y)}^{t}\left(\bar{\Pi}\left(g_{y}^{h}(s)\right)+\rho_{y}^{h}(s)\right) d s-\int_{1}^{t}(\bar{\Pi}(g(s))+\rho(s)) d s\right) .
$$

Now, by (4.1) in Lemma 4.2, uniformly in $h>0, y>g(h)$,

$$
\left|\int_{t_{0}(y)}^{\infty} \rho_{y}^{h}(s) d s\right| \lesssim \int_{t_{0}(y)}^{\infty} \frac{1}{s \log (s)^{1+\varepsilon}}\left(1+\frac{1}{f(y)-h}\right) d s,
$$

and $1 /(f(y)-h) \leq 1 / f(\delta)<\infty$ since $y \geq g(h+f(\delta))$, so the $\rho_{y}^{h}$ integral is bounded uniformly in $h>0, y>g(h+f(\delta))$. By Remark 4.5, $\int_{1}^{\infty} \rho(s) d s<\infty$. For $y>$ $g(h+f(\delta))>\delta, f(A y)>f(A \delta)$, so taking $A$ sufficiently large if necessary, $t_{0}(y):=$ 
$f(A y) \vee f(1+2 / A)=f(A y)$, then by Lemma 4.6, $\limsup _{t \rightarrow \infty} \int_{f(A y)}^{t}\left(\bar{\Pi}\left(g_{y}^{h}(s)\right)-\right.$ $\bar{\Pi}(g(s))) d s<\infty$, and so we have uniformly in $h>0, y>g(h)$,

$$
q_{h}(y) \lesssim \Phi_{y}^{h}(f(A y)) \exp \left(-\int_{1}^{f(A y)} \bar{\Pi}(g(s)) d s\right) .
$$

For the converse inequality, as $\bar{\Pi}$ is non-increasing, for $y>g(h)$ (so $f(y)>h$ ),

$$
\begin{aligned}
\int_{f(A y)}^{t}(\bar{\Pi}(g(s))-\bar{\Pi}(g(s+h)-y)) d s & \leq \int_{f(A y)}^{t}(\bar{\Pi}(g(s))-\bar{\Pi}(g(s+h))) d s \\
=\int_{f(A y)}^{t} \bar{\Pi}(g(s)) d s-\int_{f(A y)+h}^{t+h} \bar{\Pi}(g(s)) d s & \leq \int_{f(A y)}^{f(A y)+h} \bar{\Pi}(g(s)) d s \\
& \leq h \bar{\Pi}(g(f(A y))) \leq h \bar{\Pi}(y) \leq f(y) \bar{\Pi}(y) .
\end{aligned}
$$

Then as $y>g(h+f(\delta))>\delta$ and $\lim _{y \rightarrow \infty} f(y) \bar{\Pi}(y)=0$ by (3.1) (recall $\left.f^{-1}=g\right)$, we conclude

$$
q_{h}(y) \asymp \Phi_{y}^{h}(f(A y)) \exp \left(-\int_{1}^{f(A y)} \bar{\Pi}(g(s)) d s\right) .
$$

Proof of Lemma 4.9: In case (ia), with $\Pi(d x)=u(x) d x, u(x)$ has bounded decrease and bounded increase, and as $\bar{\Pi}$ is regularly varying at $\infty$ with index $-\alpha \in(-1,0)$ in case (i), it follows that $\bar{\Pi}$ has positive increase and bounded increase (see Bingham et al., 1989, p71 for precise definitions of bounded decrease, bounded increase, and positive increase). Thus we can apply Bingham et al. (1989, Prop 2.2.1), yielding that $x u(x) \asymp \bar{\Pi}(x)$ for all sufficiently large $x$, so

$$
\frac{\Pi(g(h) d v)}{\bar{\Pi}(g(h))}=\frac{u(g(h) v) g(h) d v}{\bar{\Pi}(g(h))} \asymp \frac{\bar{\Pi}(g(h) v) g(h) d v}{g(h) v \bar{\Pi}(g(h))}=v^{-1-\alpha} \frac{L(g(h) v)}{L(g(h))} d v .
$$

Proof of Lemma 4.10: For $t \geq f(A y), A>3 \vee(B-1)$, as $f$ is increasing,

$$
t \geq f(A y) \geq f(y) \geq f(y)-h .
$$

For $y>0, y>g(h)$, and $s \leq f(y)-h$, we have $g_{y}^{h}(s) \leq 0$, so

$$
\mathbb{P}\left(\mathcal{O}_{s}^{g_{y}^{h}}\right)=\mathbb{P}\left(X_{u} \geq g_{y}^{h}(u), \forall u \leq s\right) \geq \mathbb{P}\left(X_{u} \geq 0, \forall u \leq s\right)=1,
$$

and we conclude, as required, that

$$
\Phi_{y}^{h}(t)=\int_{0}^{t} \mathbb{P}\left(\mathcal{O}_{s}^{g_{y}^{h}}\right) d s \geq \int_{0}^{f(y)-h} \mathbb{P}\left(\mathcal{O}_{s}^{g_{y}^{h}}\right) d s=f(y)-h .
$$

Proof of Lemma 5.1: By Markov's inequality and (2), with $\lambda=\log (1 / H(t)) / B(t)$,

$$
\begin{aligned}
& \mathbb{P}\left(X_{t}^{(0, A(t))}>B(t)\right)=\mathbb{P}\left(e^{\lambda X_{t}^{(0, A(t))}} \geq e^{\lambda B(t)}\right) \\
& \leq \mathbb{E}\left[e^{\lambda X_{t}^{(0, A(t))}}\right] e^{-\lambda \underline{\underline{B}}(t)} \exp \left(t \int_{0}^{A(t)} \lambda e^{\lambda x}(\bar{\Pi}(x)-\bar{\Pi}(A(t))) d x\right) H(t)
\end{aligned}
$$




$$
\begin{aligned}
& \leq \exp \left(t \frac{\log (1 / H(t))}{B(t)} e^{\lambda A(t)} \int_{0}^{A(t)} \bar{\Pi}(x) d x\right) H(t) \\
& =\exp \left(t \frac{\log (1 / H(t))}{B(t)} H(t)^{-\frac{A(t)}{B(t)}} \int_{0}^{A(t)} \bar{\Pi}(x) d x\right) H(t) .
\end{aligned}
$$

Now, by Bingham et al. (1989, Theorem 2.6.1(b)), which applies as $\bar{\Pi}$ has lower index $\beta(\bar{\Pi})>-1$ in cases (i) and (ii), there exists $C>0$ such that for all $A(t)>1$,

$$
\int_{0}^{A(t)} \bar{\Pi}(x) d x \leq \int_{0}^{1} \bar{\Pi}(x) d x+C \bar{\Pi}(A(t)) A(t) .
$$

Now, consider $\mu(\bar{\Pi}):=\liminf _{x \rightarrow \infty} \log (\bar{\Pi}(x)) / \log (x)$, which satisfies $\mu(\bar{\Pi}) \geq \beta(\bar{\Pi})>$ -1 by Bingham et al. (1989, Prop 2.2.5), so $\lim \inf _{x \rightarrow \infty} \log (\bar{\Pi}(x)) / \log (x)>1$, and thus $\liminf _{x \rightarrow \infty} x \bar{\Pi}(x)>0$. So uniformly among $A(t)>1$, $\int_{0}^{1} \bar{\Pi}(x) d x \lesssim A(t) \bar{\Pi}(A(t))$, and (5.1) follows from (7.4) and (7.5), as required.

\section{Acknowledgements}

Thanks to Mladen Savov and Ronnie Loeffen for discussing this work at various stages. Further thanks to an anonymous referee for their feedback on the paper. Funding was provided by the EPSRC.

\section{References}

Aurzada, F., Kramm, T., and Savov, M. First passage times of Lévy processes over a one-sided moving boundary. Markov Process. Related Fields, 21 (1), 1-38 (2015). MR3381222.

Barker, A. Fractal-dimensional properties of subordinators. J. Theoret. Probab., 32 (3), 1202-1219 (2019). MR3979666.

Benjamini, I. and Berestycki, N. An integral test for the transience of a Brownian path with limited local time. Ann. Inst. Henri Poincaré Probab. Stat., 47 (2), 539-558 (2011). MR2814422.

Berger, Q., den Hollander, F., and Poisat, J. Annealed scaling for a charged polymer in dimensions two and higher. J. Phys. A, 51 (5), 054002, 37 (2018). MR3755571.

Bertoin, J. An extension of Pitman's theorem for spectrally positive Lévy processes. Ann. Probab., 20 (3), 1464-1483 (1992). MR1175272.

Bertoin, J. Splitting at the infimum and excursions in half-lines for random walks and Lévy processes. Stochastic Process. Appl., 47 (1), 17-35 (1993). MR1232850.

Bertoin, J. Lévy processes, volume 121 of Cambridge Tracts in Mathematics. Cambridge University Press, Cambridge (1996). ISBN 0-521-56243-0. MR1406564.

Bingham, N. H., Goldie, C. M., and Teugels, J. L. Regular variation, volume 27 of Encyclopedia of Mathematics and its Applications. Cambridge University Press, Cambridge (1989). ISBN 0-521-37943-1. MR1015093.

Biskup, M. and Louidor, O. Full extremal process, cluster law and freezing for the two-dimensional discrete Gaussian free field. Adv. Math., 330, 589-687 (2018). MR3787554.

Bogdan, K., Burdzy, K., and Chen, Z.-Q. Censored stable processes. Probab. Theory Related Fields, 127 (1), 89-152 (2003). MR2006232. 
Bolthausen, E. Large deviations and interacting random walks. In Lectures on probability theory and statistics (Saint-Flour, 1999), volume 1781 of Lecture Notes in Math., pp. 1-124. Springer, Berlin (2002). MR1915444.

Caravenna, F., den Hollander, F., and Pétrélis, N. Lectures on random polymers. In Probability and statistical physics in two and more dimensions, volume 15 of Clay Math. Proc., pp. 319-393. Amer. Math. Soc., Providence, RI (2012). MR3025394.

Çetin, U. Path transformations for local times of one-dimensional diffusions. Stochastic Process. Appl., 128 (10), 3439-3465 (2018). MR3849815.

Chaumont, L. Conditionings and path decompositions for Lévy processes. Stochastic Process. Appl., 64 (1), 39-54 (1996). MR1419491.

den Hollander, F. Der Zufall in der Chemie: Polymere. volume 79, pp. 69-77 (1999). MR1699770.

Denisov, D. and Wachtel, V. Conditional limit theorems for ordered random walks. Electron. J. Probab., 15, no. 11, 292-322 (2010). MR2609589.

Denisov, D. and Wachtel, V. Random walks in cones. Ann. Probab., 43 (3), 9921044 (2015). MR3342657.

Djurčić, D. O-regularly varying functions and strong asymptotic equivalence. $J$. Math. Anal. Appl., 220 (2), 451-461 (1998). MR1614959.

Embrechts, P., Goldie, C. M., and Veraverbeke, N. Subexponentiality and infinite divisibility. Z. Wahrsch. Verw. Gebiete, 49 (3), 335-347 (1979). MR547833.

Garbit, R. Brownian motion conditioned to stay in a cone. J. Math. Kyoto Univ., 49 (3), 573-592 (2009). MR2583602.

$\mathrm{Hu}$, Y., Khoshnevisan, D., and Wouts, M. Charged polymers in the attractive regime: a first-order transition from Brownian scaling to four-point localization. J. Stat. Phys., 144 (5), 948-977 (2011). MR2836614.

Kallenberg, O. Foundations of modern probability. Probability and its Applications (New York). Springer-Verlag, New York, second edition (2002). ISBN 0-38795313-2. MR1876169.

Kerkhoff, U. and Lerche, H. R. Boundary crossing distributions of random walks related to the law of the iterated logarithm. Statist. Sinica, 23 (4), 1697-1715 (2013). MR3222816.

Kolb, M. and Savov, M. Transience and recurrence of a Brownian path with limited local time. Ann. Probab., 44 (6), 4083-4132 (2016). MR3572332.

Kolb, M. and Savov, M. Conditional survival distributions of Brownian trajectories in a one dimensional Poissonian environment in the critical case. Electron. J. Probab., 22, Paper No. 14, 29 (2017). MR3622884.

König, W. and Schmid, P. Random walks conditioned to stay in Weyl chambers of type C and D. Electron. Commun. Probab., 15, 286-296 (2010). MR2670195.

Lerche, H. R. Boundary crossing of Brownian motion. Its relation to the law of the iterated logarithm and to sequential analysis, volume 40 of Lecture Notes in Statistics. Springer-Verlag, Berlin (1986). ISBN 3-540-96433-9. MR861122.

Mallein, B. Asymptotic of the maximal displacement in a branching random walk. Grad. J. Math., 1 (2), 92-104 (2016). MR3850767.

Nolan, J. Stable Distributions - Models for Heavy Tailed Data. Birkhauser, Boston (2018). In progress, Chapter 1 online at http://fs2.american.edu/jpnolan/ www/stable/stable.html.

Pantí, H. On Lévy processes conditioned to avoid zero. ALEA Lat. Am. J. Probab. Math. Stat., 14 (2), 657-690 (2017). MR3689384. 
Pardo, J. C. On the rate of growth of Lévy processes with no positive jumps conditioned to stay positive. Electron. Commun. Probab., 13, 494-506 (2008). MR2447836.

Pitman, J. W. One-dimensional Brownian motion and the three-dimensional Bessel process. Advances in Appl. Probability, 7 (3), 511-526 (1975). MR375485.

Pötzelberger, K. and Wang, L. Boundary crossing probability for Brownian motion. J. Appl. Probab., 38 (1), 152-164 (2001). MR1816120.

Roynette, B., Vallois, P., and Yor, M. Some penalisations of the Wiener measure. Jpn. J. Math., 1 (1), 263-290 (2006). MR2261065.

Roynette, B., Vallois, P., and Yor, M. Penalizing a $\operatorname{BES}(d)$ process $(0<d<2)$ with a function of its local time. V. Studia Sci. Math. Hungar., 45 (1), 67-124 (2008). MR2401169.

Salminen, P. and Vallois, P. On subexponentiality of the Lévy measure of the diffusion inverse local time; with applications to penalizations. Electron. J. Probab., 14, no. 67, 1963-1991 (2009). MR2540855.

van der Hofstad, R. and Klenke, A. Self-attractive random polymers. Ann. Appl. Probab., 11 (4), 1079-1115 (2001). MR1878291.

van der Hofstad, R. and König, W. A survey of one-dimensional random polymers. J. Statist. Phys., 103 (5-6), 915-944 (2001). MR1851362.

Wachtel, V. I. and Denisov, D. E. An exact asymptotics for the moment of crossing a curved boundary by an asymptotically stable random walk. Theory Probab. Appl., 60 (3), 481-500 (2016). MR3568792.

Westwater, M. J. On Edwards' model for long polymer chains. Comm. Math. Phys., 72 (2), 131-174 (1980). MR573702.

Yakymiv, A. L. On the asymptotics of the density of an infinitely divisible distribution at infinity. Teor. Veroyatnost. i Primenen., 47 (1), 80-89 (2002). MR1978697.

Yano, K., Yano, Y., and Yor, M. Penalising symmetric stable Lévy paths. J. Math. Soc. Japan, 61 (3), 757-798 (2009). MR2552915. 